\title{
Optical spectroscopy of EX Lupi during quiescence and outburst
}

\section{Infall, wind, and dynamics in the accretion flow ${ }^{\star}$}

\author{
A. Sicilia-Aguilar ${ }^{1}$, Á. Kóspál ${ }^{2,3, \star \star}{ }^{\text {, J. Setiawan }}{ }^{4}$, P. Ábrahám ${ }^{5}$, C. Dullemond ${ }^{4,6}$, C. Eiroa ${ }^{1}$, M. Goto ${ }^{4}$, \\ Th. Henning ${ }^{4}$, and A. Juhász ${ }^{2}$
}

${ }^{1}$ Departamento de Física Teórica, Facultad de Ciencias, Universidad Autónoma de Madrid, 28049 Cantoblanco, Madrid, Spain e-mail: aurora.sicilia@uam.es

${ }^{2}$ Leiden Observatory, Leiden University, PO Box 9513, 2300 RA Leiden, The Netherlands

3 Research and Scientific Support Department, European Space Agency, ESA/ESTEC, SRE-SA, PO Box 299, 2200 AG, Noordwijk, The Netherlands

4 Max-Planck-Institut für Astronomie, Königstuhl 17, 69117 Heidelberg, Germany

5 Konkoly Observatory, Research Centre for Astronomy and Earth Sciences, Hungarian Academy of Sciences, PO Box 67, 1525 Budapest, Hungary

${ }^{6}$ Institut für Theoretische Astrophysik, Zentrum für Astronomie, Universität Heidelberg, Albert-Ueberle-Str. 2, 69120 Heidelberg, Germany

Received 1 December 2011 / Accepted 7 June 2012

\begin{abstract}
Context. EX Lupi is the prototype of EXor variables. After 50 years of mild variability and smaller outbursts, the object again experienced a large outburst in 2008.

Aims. We explore the accretion mechanisms in EX Lupi during its pre-outburst, outburst, and post-outburst phases.

Methods. We analyze 10 high-resolution optical spectra of EX Lupi, taken before, during, and after the 2008 outburst. In both quiescence and outburst, the star presents many permitted emission lines. These include lines typical of accreting T Tauri stars, plus a large number of neutral and ionized metallic lines (mostly Fe I and Fe II). During the outburst, the number of emission lines increases to about a thousand, and their structure shows a narrow and a broad component (NC and BC). We study the structure of the BC, which is highly variable on short timescales (24-72 h).

Results. An active chromosphere can explain the metallic lines in quiescence and the outburst NC. The dynamics of the BC line profiles suggest that these profiles originate in a hot, dense, non-axisymmetric, and non-uniform accretion column that suffers velocity variations along the line-of-sight on timescales of days. Assuming Keplerian rotation, the emitting region would be located at $\sim 0.1-0.2 \mathrm{AU}$, which is consistent with the location of the inner disk rim, but the velocity profiles of the lines reveal a combination of rotation and infall. Line ratios of ions and neutrals can be reproduced assuming a temperature of $T \sim 6500 \mathrm{~K}$ for electron densities of a few times $10^{12} \mathrm{~cm}^{-3}$ in the line-emitting region. The line profiles also indicate that there is an accretion-related inner disk wind. Conclusions. The data confirm that the 2008 outburst was an episode of increased accretion, albeit much stronger than previous EX Lupi and typical EXors outbursts. The line profiles are consistent with the infall/rotation of a non-axisymmetric structure that could be produced by clumpy accretion during the outburst phase. A strong inner disk wind appears in the epochs of higher accretion. The rapid recovery of the system after the outburst and the similarity between the pre-outburst and post-outburst states suggest that the accretion channels are similar during the whole period, and only the accretion rate varies, providing a superb environment for studying the accretion processes.
\end{abstract}

Key words. line: profiles - stars: variables: T Tauri, Herbig Ae/Be - stars: individual: EX Lupi - stars: pre-main sequence protoplanetary disks - stars: low-mass

\section{Introduction}

EX Lupi is a young, low-mass star (spectral type M0; Herbig 1977; Gras-Velázquez \& Ray 2005), prototype of the EXor family among young variable T Tauri stars (Herbig 1989). Its irregular variability has been known for about 120 years (McLaughlin 1946), although the causes of these variations and their association with young stars were not suggested until much later (Herbig 1950). While the normal variability pattern is characterized by irregular fluctuations, with small outbursts where the brightness increases by $\sim 2.5$ mag over timescales of months to few years, a much stronger outburst was observed in 1955-57

\footnotetext{
* Appendix A and Tables 2 and 3 are available in electronic form at http://www . aanda.org

$\star \star$ ESA Fellow.
}

(Herbig 1977). This episode was followed by more than 50 years of small-scale variability, until in January 2008 another large outburst was observed (Jones 2008). Spectroscopic observations of EX Lupi in outburst and during its quiescence phases revealed that it is a M0 classical T Tauri star (CTTS) that suffers episodes of variable mass accretion (Herbig et al. 2001). The larger outbursts could be assimilated to episodes of largely increased mass infall, up to three orders of magnitude over the typical quiescence levels (Juhász et al. 2012; Kóspál et al. 2011).

The 2008 outburst offered a remarkable opportunity to check the hypothesis of increased accretion episodes, and to study the causes of mass infall and its effect on the disk. The outburst was characterized by a rapid rise from the quiescence state in November 2007, to a maximum around January 2008 (Jones 2008). It was followed by a slow decay until it reached 
Table 1. Journal of observations and estimated magnitudes.

\begin{tabular}{lcccccc}
\hline \hline Date & JD & Instrument & Exp. time $(\mathrm{s})$ & $S / N$ & Magnitude & Comments \\
\hline 2007 July 28 & 2454309.114 & FEROS & 1600 & $0.7 / 30 / 28$ & $12.7:{ }^{1}$ & Pre-outburst \\
2007 July 29 & 2454310.156 & FEROS & 1800 & $0.3 / 30 / 20$ & $12.7:{ }^{1}$ & Pre-outburst \\
2007 July 30 & 2454311.187 & FEROS & 1800 & $0.2 / 15 / 14$ & $12.7:{ }^{1}$ & Pre-outburst \\
2008 April 21 & 2454577.277 & FEROS & 2400 & $13 / 220 / 65$ & $9.55^{2}$ & Outburst \\
2008 May 5 & 2454591.426 & FEROS & 2000 & $1 / 46 / 23$ & $9.7:^{1}$ & Outburst \\
2008 May 6 & 2454592.152 & FEROS & 2000 & $5 / 90 / 36$ & $9.7: 1^{1}$ & Outburst \\
2008 May 8 & 2454594.360 & HARPS & 2000 & $-/ 32 /-$ & $9.7^{1}$ & Outburst \\
2008 June 16 & 2454633.084 & FEROS & 2000 & $8 / 165 / 51$ & $9.6^{1}$ & Outburst \\
2010 June 9 & 2455356.339 & FEROS & 2000 & $0.1 / 13 / 8$ & $13.7^{1}$ & Post-outburst \\
2010 June 11 & 2455358.271 & FEROS & 2000 & $0.2 / 10 / 8$ & $13.7: 1$ & Post-outburst \\
\hline
\end{tabular}

Notes. Information on the spectroscopy observations. The signal-to-noise ratio $(\mathrm{S} / \mathrm{N})$ is measured in three different parts of the spectra $(\sim 3650$, $\sim 6700$, and $\sim 8800 \AA$ ). The magnitudes are visual data from AAVSO (marked with ${ }^{(1)}$ ) and $V$ magnitude taken with WFI at the $2.2 \mathrm{~m}$ telescope in La Silla (marked with ${ }^{(2)}$; Juhász et al. 2012). If no data was obtained on the given day, we interpolated from nearby observations and indicate interpolated observations with ":". Variations in the S/N between spectra taken at similar brightness of the source are due to weather conditions. The pre-outburst data were presented in Sipos et al. (2009).

quiescence levels again by September 2008. During this decrease, the optical magnitude of the object suffered a few oscillations with amplitudes 1-2 mag (increasing with time) and period of about 35 days (Juhász et al. 2012). During the 2008 outburst, the optical spectrum of EX Lupi became full of broad metallic emission lines, with hardly any photospheric absorption line remaining visible (Kóspál et al. 2008; Aspin et al. 2010). This was an outstanding behavior, given that the emission lines observed in previous, weaker outbursts were rather narrow and more similar to the emission lines observed during quiescence (Herbig et al. 2001; Lehmann et al. 1995). The brightening of the star also affected the disk, allowing a real-time experiment on silicate processing and crystallization as well as mixing within the disk (Ábrahám et al. 2009; Juhász et al. 2012) that suggested the development of a disk wind during the outburst phase. CO observations uncovered the rotational pattern of the emitting gas (Goto et al. 2011), which is consistent with gaseous material filling in the inner hole of the dust disk that had been suggested by observations of the object in quiescence (Sipos et al. 2009). These observations were consistent with the results of additional near infrared (near-IR) spectroscopy data, which revealed the presence of a $\sim 0.1-0.3 \mathrm{AU}$ dust-free region in the disk, where gas may be located at the edge of the disk and/or in the outer part of a funnel flow (Kóspál et al. 2011).

Here we present and analyze a collection of ten optical spectra of EX Lupi taken during the pre-outburst, 2008 outburst, and post-outburst phases. Optical spectroscopy traces the innermost structure in the system, being the key to understanding the underlying physics of the EXor outburst processes. It allows us to determine the differences in accretion, chromospheric emission, and innermost gaseous disk structure between the quiescence and the outburst phase. In Sect. 2, we describe the observations and data reduction. In Sect. 3, we study the emission features observed in quiescence (before and after the outburst) and during the outburst. In Sect. 4, we explore the physical processes and structures that can be related to the optical emission lines, including accretion, wind, and chromospheric activity. We finally summarize our results in Sect. 5.

\section{Observations and data reduction}

A total of 10 spectra were taken with the Fiber-fed Extended Range Optical Spectrograph (FEROS), mounted on the 2.2 m Max-Planck Gesellschaft/European Southern Observatory
(MPG/ESO) telescope, and the High Accuracy Radial velocity Planet Searcher (HARPS), mounted on the $3.6 \mathrm{~m}$ telescope (both located at ESO-La Silla observatory) during MPG guaranteed time. FEROS has a resolution of $R=48000$ and a wavelength coverage from 3700-9200 ̊ (Kaufer et al. 2000). HARPS has a similar resolution, but a shorter wavelength coverage (3800-6900 ̊). The observations took place during 2007, 2008, and 2010. Three of them correspond to the pre-outburst phase (2007 July 28, 29, and 30), five were taken close to the maximum of the 2008 outburst and in the subsequent months (on 2008 April 21, May 5, 6, and 8, June 16), and two more were obtained in 2010, again during a quiescent phase of EX Lupi (2010 June 9, 11). A journal of the observations is presented in Table 1. The AAVSO reports visual magnitudes of 12.7 for the pre-outburst observations (extrapolating from measurements in the previous week), 10.1 for 2008 April 21 (with our WFI observations giving $V=9.554 \pm 0.003$, see Juhász et al. 2012), 9.7 and 9.6 for 2008 May 8 and 2008 June 16, with similar values extrapolating to 2008 May 5 and 6, and a magnitude of 13.7 on 2010 June 9. Therefore, the star was slightly brighter than its minimum during our pre-outburst observations, and reached its typical minimum magnitude at the time the postoutburst spectra were taken. The spectral reduction was performed using the online data reduction pipeline installed at the telescope. This pipeline produced a one-dimensional spectrum from $370-920 \mathrm{~nm}$, generated by merging 39 echelle-order spectra. The line analysis (including measurements of wavelength, equivalent width [EW], and integrated intensity) was done with standard tasks within the IRAF ${ }^{1}$ onedspec package.

\section{Analysis}

Young stars such as CTTS and Herbig Ae/Be (HAeBe) stars are characterized by the presence of numerous emission lines in their spectra (see for instance Appenzeller et al. 1986; Hamann \& Persson 1992a,b; Hamann 1994). Many of the emission lines observed in EX Lupi are common among young T Tauri stars (TTS) and HAeBe stars, although the large number of metallic emission lines found in EX Lupi (both in quiescence and

1 IRAF is distributed by the National Optical Astronomy Observatories, which are operated by the Association of Universities for Research in Astronomy, Inc., under cooperative agreement with the National Science Foundation. 
outburst) are rare among typical CTTS, having precedents only in the observations of some very active CTTS and other EXors (Appenzeller et al. 1986; Hamann \& Persson 1992a; Beristain et al. 1998; Parsamian \& Mujica 2004; Herbig 2008). The commonly observed permitted lines include those of $\mathrm{H}$ I emission (especially the Balmer series dominated by $\mathrm{H} \alpha$ ), the Ca II IR triplet, and He I emission, all of which are typically related to the accretion activity. In some cases, some O I lines and the $\mathrm{Na}$ I doublet appear as well in emission. Forbidden lines (e.g. [O I], [S II]) are also a common occurrence in CTTS and HAeBe (Hamann 1994), although EX Lupi does not display any evidence of them during either quiescence or outburst, confirming the results of Kóspál et al. (2011) in the near-IR. This suggests that, despite the wind signatures we describe later, there is no shock or it is quenched (Nisini et al. 2005). Therefore, the environment around EX Lupi seems to be clean of cloud material and remnant protostellar envelopes, in agreement with model predictions (Sipos et al. 2009).

The observed emission lines are listed in Table 2 (for the quiescence phase) and Table 3 (for the outburst phase). The lines were identified using the National Institute of Standards and Technology (NIST) database ${ }^{2}$ (Ralchenko et al. 2010), which includes information about the wavelength, excitation potential (or energy of the upper level, $E_{k}$ ), multiplicity, relative intensity, and transition probabilities (or Einstein coefficients, $A_{k i}$ ). A few lines could not be identified in the NIST database, being thus listed as "INDEF" in our Tables. Some of the lines that do not appear in the NIST database are listed in Appenzeller et al. (1986), in which cases we adopted their classification. In the following sections, we analyze the observed lines during the quiescence and outburst phases.

\subsection{The quiescence phases: pre- and post-outburst}

During the quiescence phases, EX Lupi displayed the photospheric absorption lines characteristic of a late-type star, together with several broad and narrow emission lines (see Sipos et al. 2009 and Table 2). There were no significant differences between the metallic lines observed in the pre-outburst and postoutburst quiescence phases, although the post-outburst spectra have poorer signal-to-noise ratio $(\mathrm{S} / \mathrm{N})$, which resulted in fewer lines being clearly identified. Only the accretion-related lines (H I Balmer series, He I, Ca II) display significant epoch and day-to-day variations.

The H I Balmer series in emission is the most remarkable feature, with $\mathrm{H} \alpha$ being the strongest line in the spectra (Fig. 1). The $\mathrm{H} \alpha$ line appears to be asymmetric, with redshifted absorption that was most prominent during the pre-outburst phase and caused the peak of the line to appear blueshifted by about $20 \mathrm{~km} \mathrm{~s}^{-1}$. Redshifted absorptions signatures of infall that are commonly seen in CTTS, and had also been observed during the 1993 EX Lupi outburst (Lehmann et al. 1995), when $\mathrm{H} \alpha$ displayed an inverse P-Cygni profile. The day-to-day variations in the $\mathrm{H} \alpha$ emission line suggest that there was a mild variation in the accretion rate. The line wings extend beyond $\pm 200 \mathrm{~km} \mathrm{~s}^{-1}$, with the red part being in general broader. The higher Balmer lines are more symmetric and less variable, with the redshifted absorption being present to a lesser extent. They have wings extending beyond $\pm 200 \mathrm{~km} \mathrm{~s}^{-1}$. A small blueshifted absorption centered at $\sim-250 \mathrm{~km} \mathrm{~s}^{-1}$ is seen in $\mathrm{H} \beta$, which is an indication of a weak cool wind.

\footnotetext{
${ }^{2}$ http://physics.nist.gov/PhysRefData/ASD/lines_form. html
}
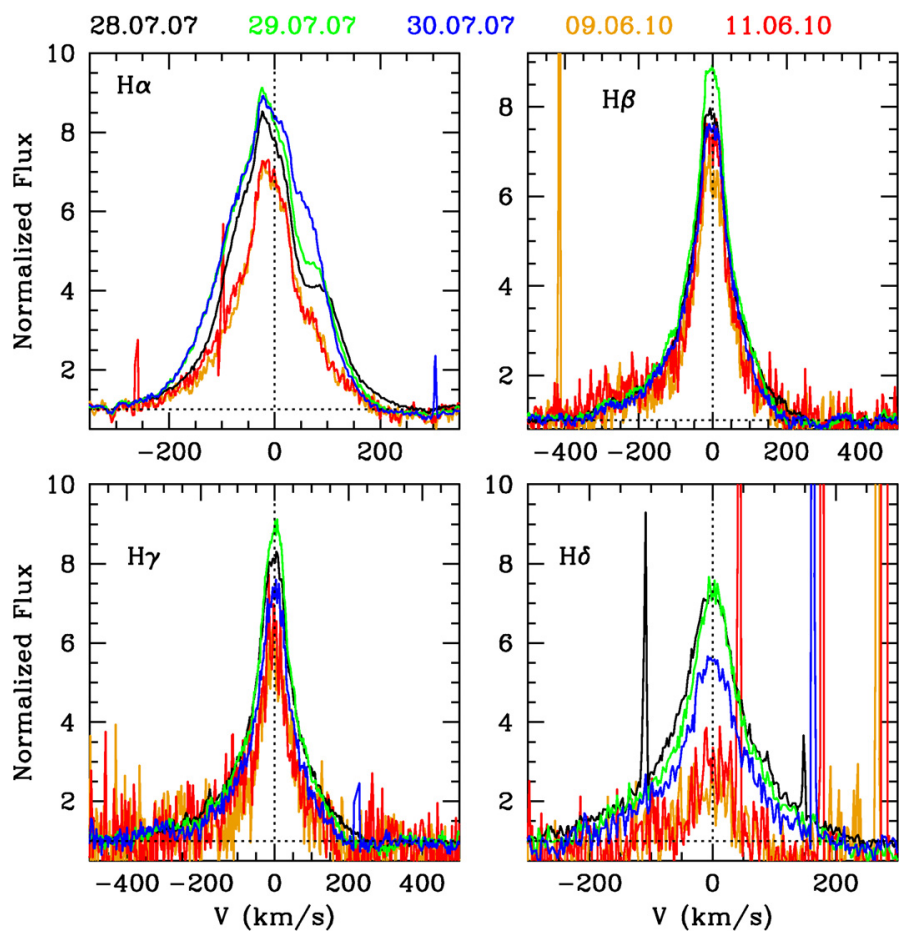

Fig. 1. H lines during the quiescence phases (pre- and post-outburst). The zero velocity is marked by a dotted line. Different colors represent the observations on different dates (black for 2007 July 28, green for 2007 July 30, blue for 2007 July 30, orange for 2010 June 9, and red for 2010 June 11).

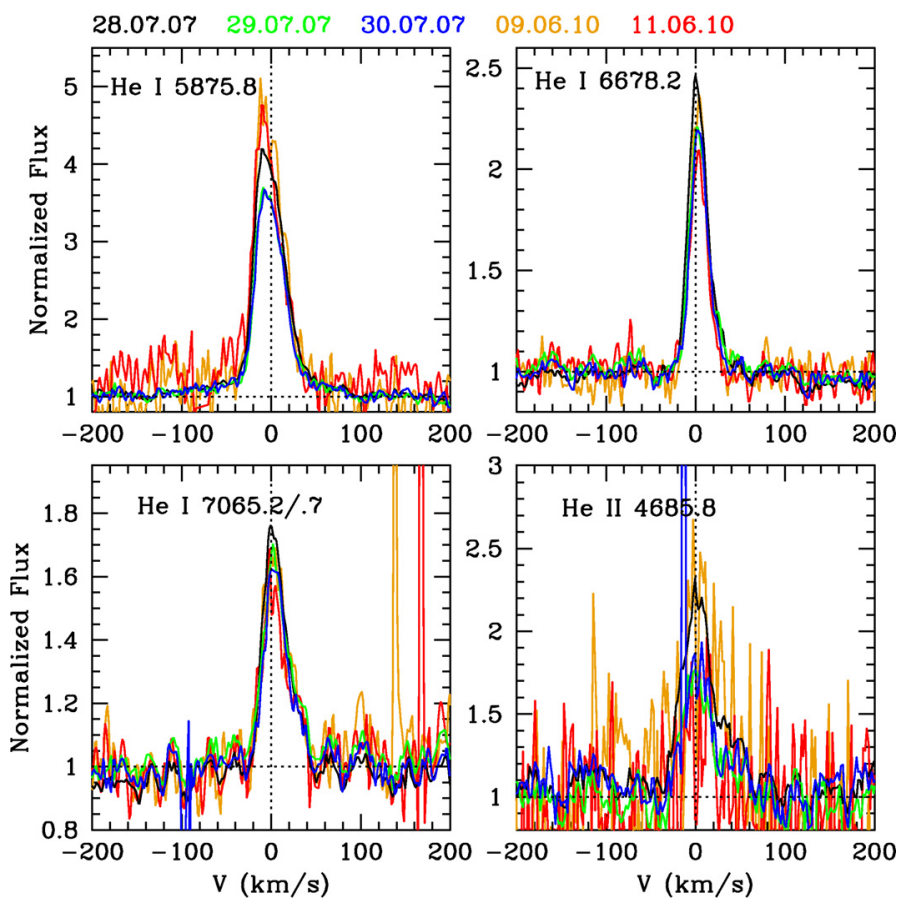

Fig. 2. He lines during the quiescence phases (pre- and post-outburst). The zero velocity is marked by a dotted line. Different colors mark different dates as in Fig. 1.

The quiescence spectra also display strong He I emission at 5878, 6678, and $7065 \AA$ (Fig. 2). The lines are narrow, with a full width at half maximum (FWHM) of $\sim 50 \mathrm{~km} \mathrm{~s}^{-1}$ and asymmetric, with a stronger red component, and mild variability in both profile and intensity. Given the high temperatures and densities required for the formation of He I lines, they probably 

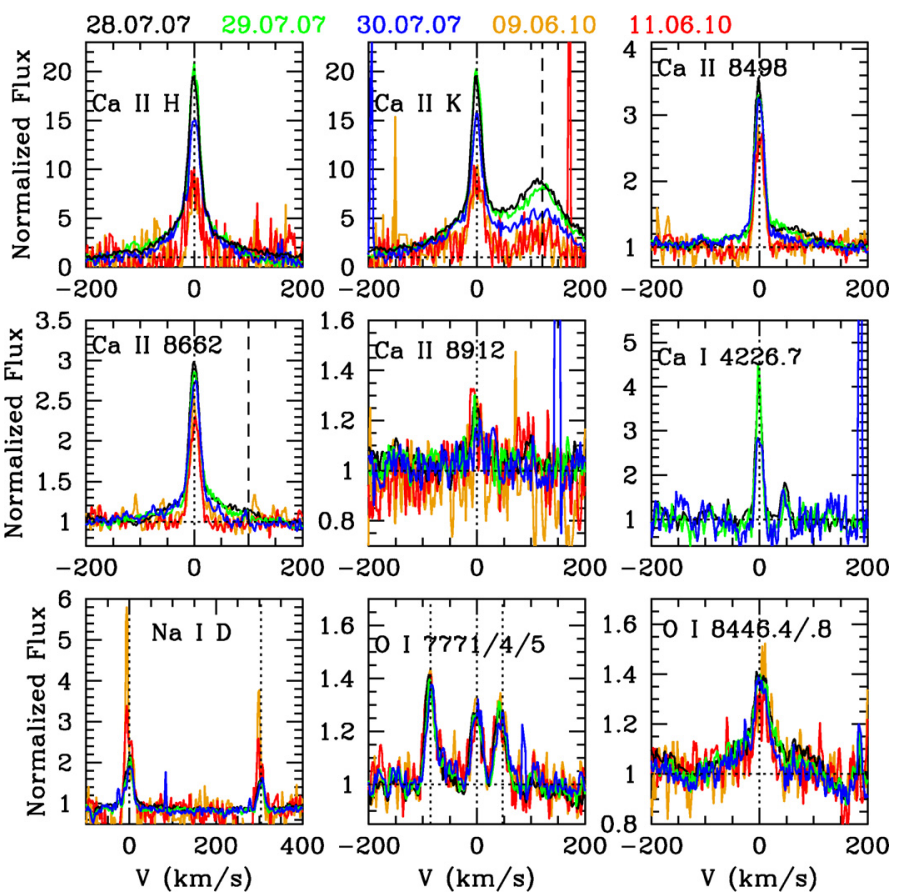

Fig. 3. Some of the Ca I, Ca II, Na I, and O I lines seen in emission during the quiescence phases (pre- and post-outburst). The zero velocity is marked by a dotted line (in the cases of the Na I D and O I triplet, we mark the zero velocity for each resolved line). The dashed lines in the Ca II K and Ca II 8662 A lines mark the position of the Balmer H $\epsilon$ and Paschen 13 lines, respectively. The weaker line redwards of Ca I 4226 is Fe I 4227. Different colors mark different dates as in Fig. 1.

originate in the densest and hottest parts of the accretion column, close to the star, and in the accretion shock itself (Hamann \& Person 1992a; Berinstain et al. 2001). The He II $4686 \AA$ line is also detected, as noted by Herbig (2007) during other epochs. He II lines require extremely hot conditions for their production, so that they are rare even in the proximity of $\mathrm{O}$ stars, and more typical of novae, Wolf-Rayet stars, and planetary nebulae (Torres \& Massey 1987; Mason et al. 2010).

The Ca II H and $\mathrm{K}$ lines, together with the Ca II IR triplet ${ }^{3}$, are also observed in emission (see Fig. 3). The Ca II lines have wings extending to $\pm 100 \mathrm{~km} \mathrm{~s}^{-1}$ and display a weak asymmetric profile consistent with infall. The $\mathrm{Na} I \mathrm{D}$ is also observed in emission, with a weak blueshifted absorption, and the $\mathrm{O}$ I triplet at 7771/4/5 $\AA$ and O I line at $8446 \AA$ also appear in emission.

Several Fe I and Fe II lines are observed in emission (Fig. 4), as noted in previous weaker outbursts (Lehmann et al. 1995; Herbig et al. 2001). Herbig (2007) reported the presence of Fe I, Fe II, Ti II, and other narrow emission lines during the quiescence period around 2000. We confirm all these lines in our data and, in addition, we observe some Ca I, Cr I, Cr II, Mg I, Si I, Si II, Mn I, and Co I emission lines. The neutral metallic lines appear frequently superimposed on the corresponding photospheric absorption lines, resembling in some cases doublepeaked absorption lines due to the weak and narrow emission component in the center. The spectral resolution does not allow us to study in great detail the line profiles, which appear symmetric and narrow $\left(F W H M \sim 10-20 \mathrm{~km} \mathrm{~s}^{-1}\right)$. The emission lines observed in the post-outburst phase tend to have lower $E_{k}$ than those observed in the pre-outburst, which could be indicative of

\footnotetext{
3 The Ca II line at $8542 \AA$ falls at the edge of the FEROS gap, so is not visible in quiescence and only partially visible during the outburst phase. We therefore excluded it from the figures.
}
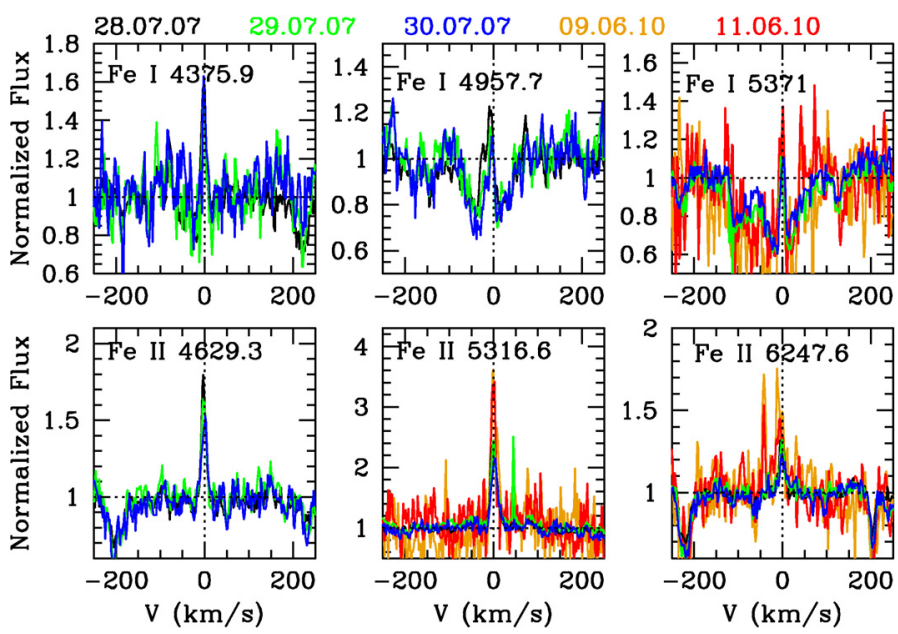

Fig. 4. Some of the Fe I and Fe II lines seen in emission during the quiescence phases (pre- and post-outburst). The zero velocity is marked by a dotted line. Different colors mark different dates as in Fig. 1.

a mild change in the temperature of the emitting region towards lower temperatures (maybe due to a lower accretion rate), although the poorer $\mathrm{S} / \mathrm{N}$ in the post-outburst may increase the difficulty of the identification of the weakest lines.

\subsection{The outburst}

During the outburst phase, the spectrum became a forest of strong emission lines (Kóspál et al. 2008) with large daily variations in the lines and continua. The lines correspond to the same species detected in quiescence, but the number of neutral and ionized permitted metallic lines increased from $\sim 150$ in quiescence, to several hundreds and probably over a thousand in outburst $^{4}$ with typical intensities that were 10-100 times higher. All the emission lines, except $\mathrm{H} \mathrm{I}$, contain a broad and a narrow component (BC and $\mathrm{NC}$ ). Composite $\mathrm{NC}+\mathrm{BC}$ line profiles have been reported in previous outbursts (Herbig 2007), as well as during the earliest phases of the 2008 outburst (Aspin et al. 2010), although most CTTS show either broad or narrow components (Hamann \& Persson 1992a). The lines reported by Herbig (2007) for the 1998 outburst had inverse P-Cygni profiles, which are typical of infall. The lines observed here show rapidly variable and complex signatures, both blueshifted and redshifted, as we later discuss. The BCs typically appear to be blended due to the number of broad lines. Therefore, the line identification during the outburst phase was always done based on the NCs, with widths $\sim 10-20 \mathrm{~km} \mathrm{~s}^{-1}$. The NCs always appear at rest velocity $\left(\sim 0 \pm 3 \mathrm{~km} \mathrm{~s}^{-1}\right)$, do not suffer strong variations in their profile (although they may vary in strength), and are thus similar, albeit stronger, to the lines observed in quiescence. Table 4 lists the line parameters for the handful of lines that do not suffer strong blending.

The very veiled Li I $6708 \AA$ line (Fig. 5) is the only one seen in absorption in the outburst spectra, although it also appears to contain some weak BC emission. All photospheric lines typical of low-mass stars (Fe I, Ca I, Mg I, K I) are seen in emission. In addition, we see the accretion-related lines typical of CTTS (H I Balmer and Paschen series, He I, Ca II IR triplet) together with ionized lines that are common in cases of strong winds and novae (Fe II, Cr II, Ti II; Mason et al. 2010) and C I lines (Hamann \& Persson 1992a,b).

\footnotetext{
4 We estimate that a similarly large number of lines to those indicated in Table 3 could not be identified owing to blends with nearby features.
} 


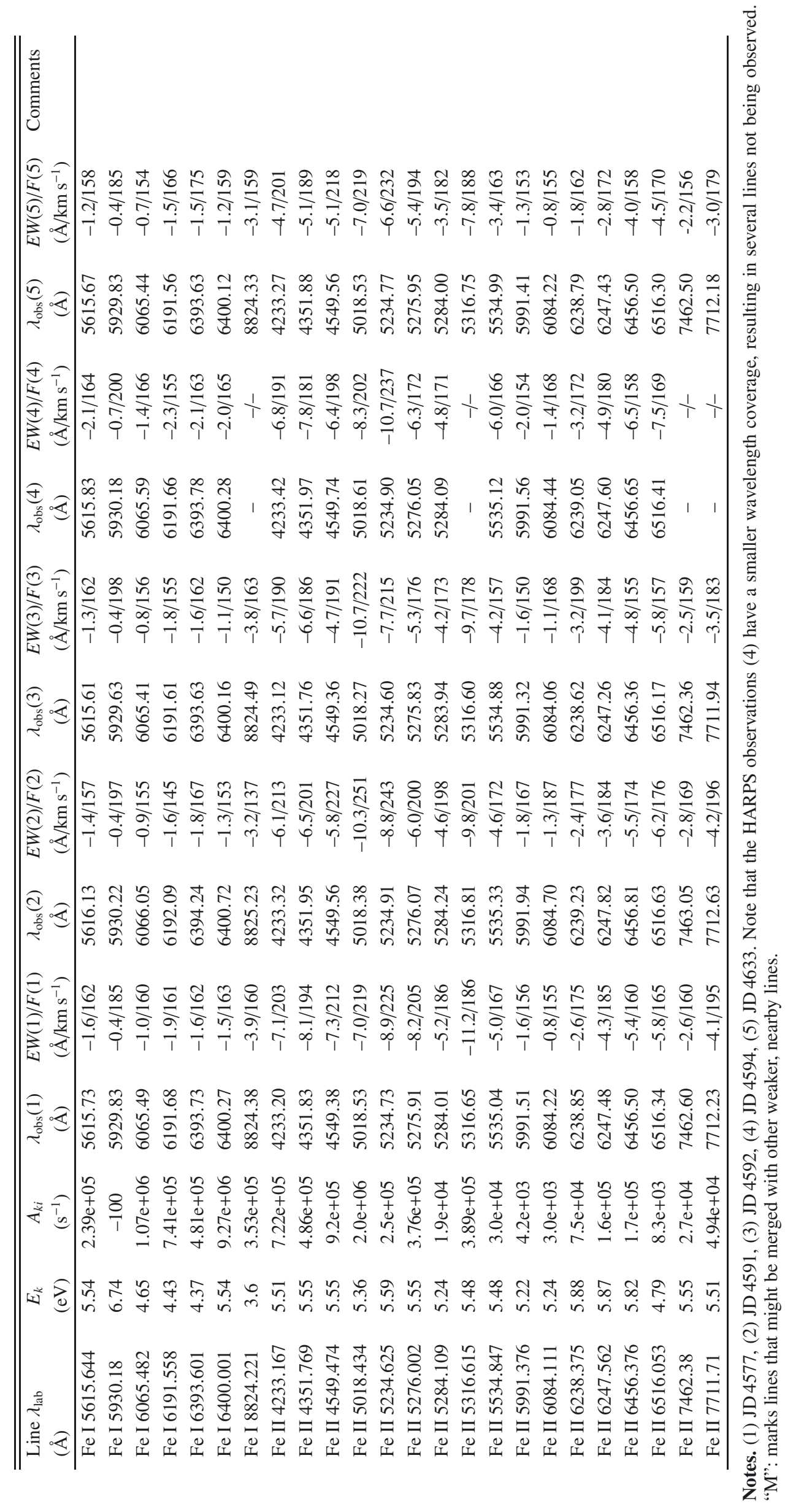




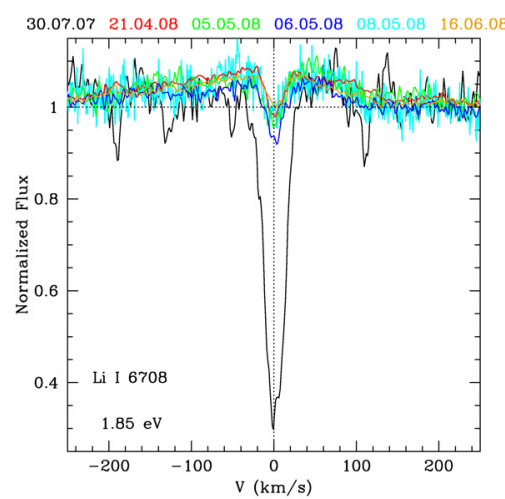

Fig. 5. Li I 6708 Å line, the only one that still appears in absorption during the outburst phase. Colors mark the observations at different dates (red for 2008 April 21, green for 2008 May 5, blue for 2008 May 6, cyan for 2008 May 8, orange for 2008 June 16, and black for the scaled spectrum in quiescence).
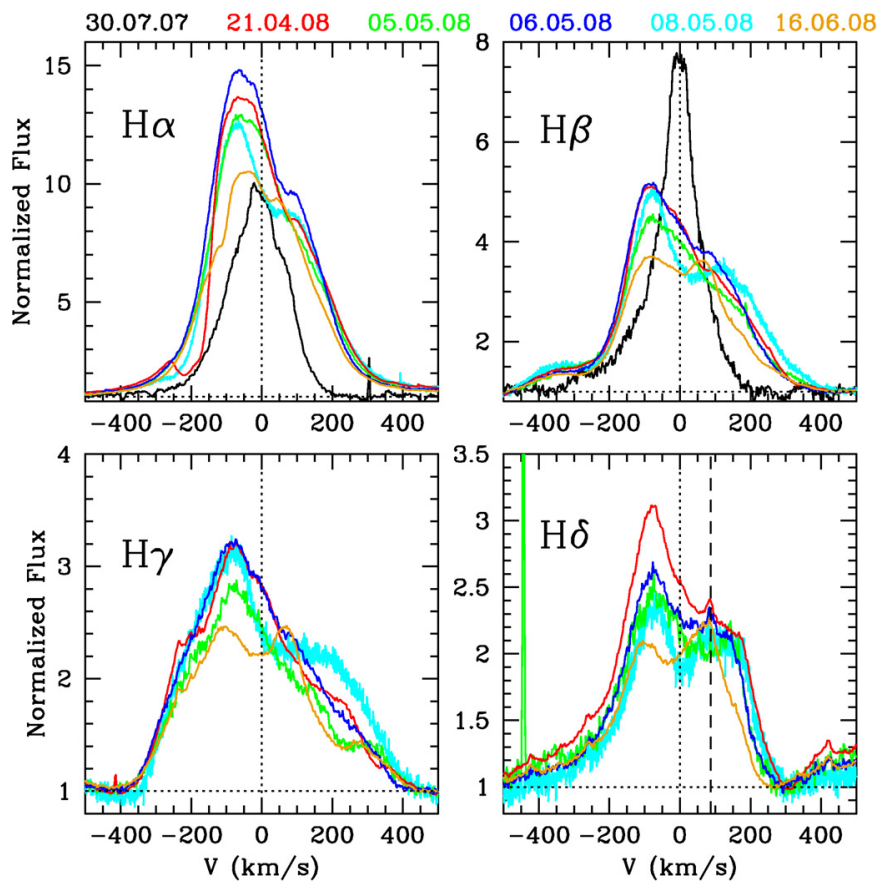

Fig. 6. Some $\mathrm{H}$ lines during the outburst phase. The zero velocity is marked by a dotted line. For comparison, the scaled quiescence spectrum of 2007 July 30 is displayed (except for $\mathrm{H} \gamma$ and $\mathrm{H} \delta$, owing to low $\mathrm{S} / \mathrm{N})$. The color code is as in Fig. 5.

The $\mathrm{H} \alpha$ emission line is the strongest and broadest one in the spectra, followed by the rest of the $\mathrm{H}$ I Balmer series (Fig. 6) and other typical accretion-related features ( $\mathrm{Ca}$ II $\mathrm{H}$ and $\mathrm{K}$ lines, Ca II IR triplet; see Fig. 7). The broad line wings extend beyond $\pm 400 \mathrm{~km} \mathrm{~s}^{-1}$ (for the $\mathrm{H} \mathrm{I}$ Balmer series) and $\pm 200 \mathrm{~km} \mathrm{~s}^{-1}$ (in the other accretion-related lines). No $\mathrm{NC}$ is visible in the $\mathrm{H}$ lines. $\mathrm{H} \alpha$ and $\mathrm{H} \beta$ display a redshifted absorption feature centered on $\sim+20-50 \mathrm{~km} \mathrm{~s}^{-1}$, while the structures of the $\mathrm{H} \gamma$ and $\mathrm{H} \delta$ lines are more complex, and vary from redshifted absorption in most observations, to nearly double-peaked lines in June. $\mathrm{H} \alpha$ and $\mathrm{H} \beta$ also display strong blueshifted absorption at $-200--250 \mathrm{~km} \mathrm{~s}^{-1}$ that can be identified with an accretionrelated wind. The blueshifted absorption varies with time, decreasing in parallel with the accretion rate from April to June, which is consistent with the general picture of mass loss related to the accretion processes in young stars (e.g. Shu et al. 1994; Calvet et al. 1997). The observations of Aspin et al. (2010)
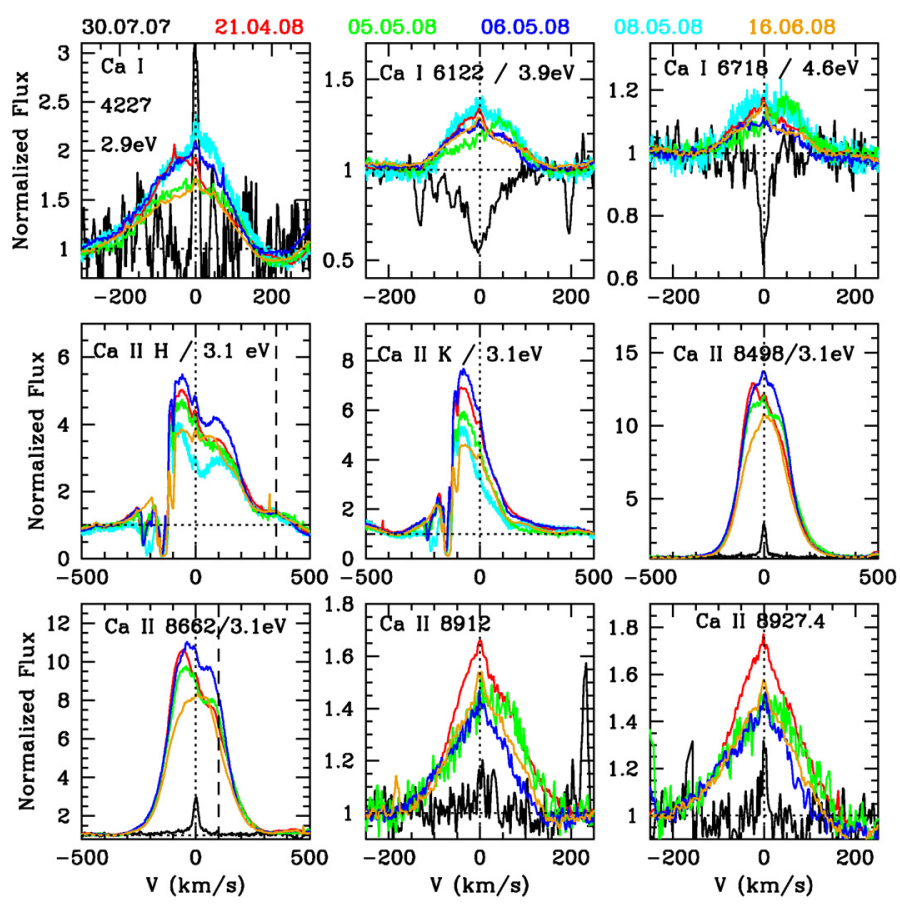

Fig. 7. Some of the Ca I and Ca II lines observed in emission during the outburst phase. The zero velocity is marked by a dotted line. In the Ca II H panel, we also indicate with a dashed line the Fe II $3938.3 \AA$ line $(4.81 \mathrm{eV})$, and note that the redshifted emission may be contaminated by more Fe I and Fe II lines in this wavelength range. For comparison, the quiescence spectrum of 2007 July 30 is displayed. The color code is as in Fig. 5.
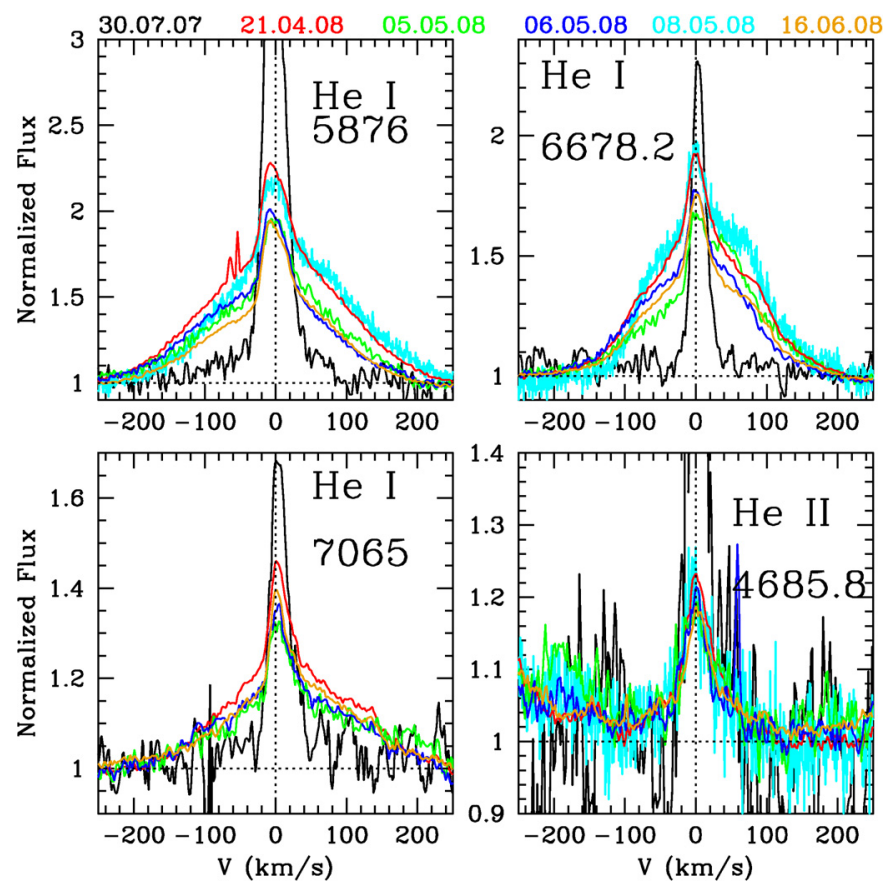

Fig. 8. Some of the He lines observed during the outburst phase. The zero velocity is marked by a dotted line. For comparison, the quiescence spectrum of 2007 July 30 is displayed. The color code is as in Fig. 5.

also confirm that there was a decrease in the strength of the blueshifted absorption as the outburst faded.

Several He I lines are also observed (Fig. 8). The high temperatures and densities required to excite the He I transitions suggest that they are produced at the bottom of the accretion column. Nevertheless, we observe a $\mathrm{BC}+\mathrm{NC}$ structure in the 

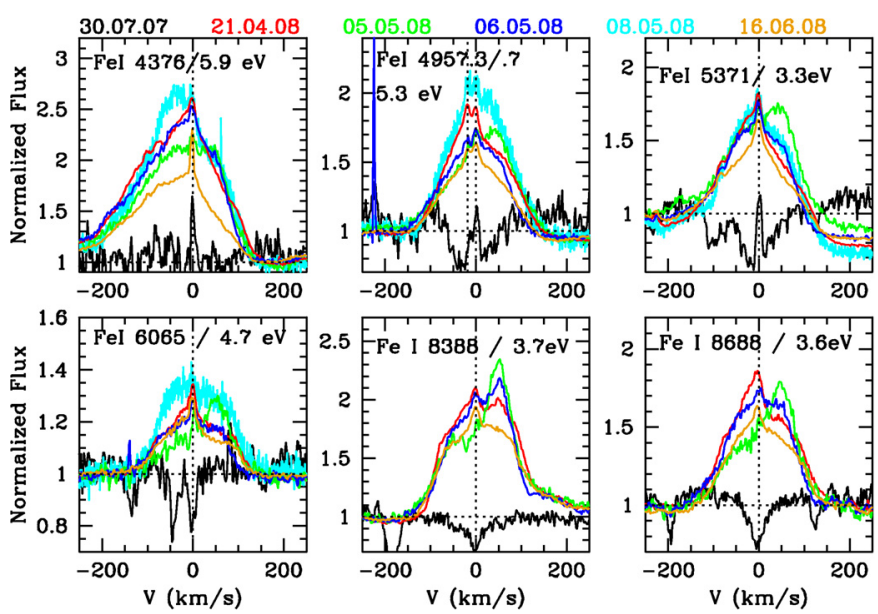

Fig. 9. Some of the Fe I lines observed in emission during the outburst phase. The zero velocity is marked by a dotted line. For comparison, the quiescence spectrum of 2007 July 30 is displayed. The color code is as in Fig. 5.

He I lines, which indicates that these lines may have two different origins (e.g. chromosphere and accretion column). The $\mathrm{BC}$ of the He I lines are asymmetric, with flattened wings that give them a triangular appearance, which differs from the profiles observed in $\mathrm{H} \mathrm{I}$ and in the metallic lines. The $\mathrm{NC}$ in the He I lines is broader $\left(\sim 40 \mathrm{~km} \mathrm{~s}^{-1}\right)$ than the typical NC observed in the metallic lines, which has been suggested as proof that the lines originate in a transition zone between the chromosphere and corona, rather than have a chromospheric origin (Hamann \& Persson 1992a). He II emission at $4686 \AA$ is also observed. The $\mathrm{He}$ II line is relatively narrow and similar to the He I NC, with which it probably shares a physical location.

The most abundant neutral metallic lines observed in emission in the outburst are, as in quiescence, those of Fe I (see Fig. 9). There are no strong Fe I lines with $E_{k}>7 \mathrm{eV}$, with most of the lines having $E_{k} \sim 3.5-6.8 \mathrm{eV}$, in agreement with the results of Kóspál et al. (2011) for the metallic lines in the near-IR spectrum. The BC of the Fe I lines are very asymmetric. Close to the outburst maximum in April, the lines tend to have two absorption components, one that is blueshifted $\left(\sim-50 \mathrm{~km} \mathrm{~s}^{-1}\right)$ and another that is redshifted (broader, resulting in the steep profile in the red part of the line), which could be the signature of wind and infall, respectively (see for instance Fe I 4376 in Fig. 9). The line profiles are similar, although have a stronger $\mathrm{NC}$, a stronger redshifted absorption, and weaker or absent blueshifted absorption, in June 16 (with profiles reminiscent of high-inclination magnetospheric infall; Hartmann et al. 1994). During the May 2008 observations, we observe strong dynamical changes in the Fe I line profiles that are not correlated with the accretion rate and have timescales of 1-2 days. The BCs appear strongly redshifted on 2008 May 5, and the redshifted emission subsequently decreases (2008 May 6) until both the redshifted and the blueshifted part of the line are roughly equal or even blue-dominated (by 2008 May 8). This is consistent with the double-peaked lines reported by Aspin et al. (2010), although our more complete coverage suggests that the double peaks are a dynamical effect rather than due to the particular geometry of a static structure. Remarkably, although there is some similarity to the Fe lines observed by Beristain et al. (1998) in DR Tau, which also display a $\mathrm{NC}+\mathrm{BC}$ structure, the dynamics observed here are in strong contrast to the stable velocities observed in DR Tau.

Other neutral lines observed include Ca I, Co I, Si I, Cr I, Ni I, Mg I, Ti I, and V I (see Figs. 7, 10, 11). The NC of the
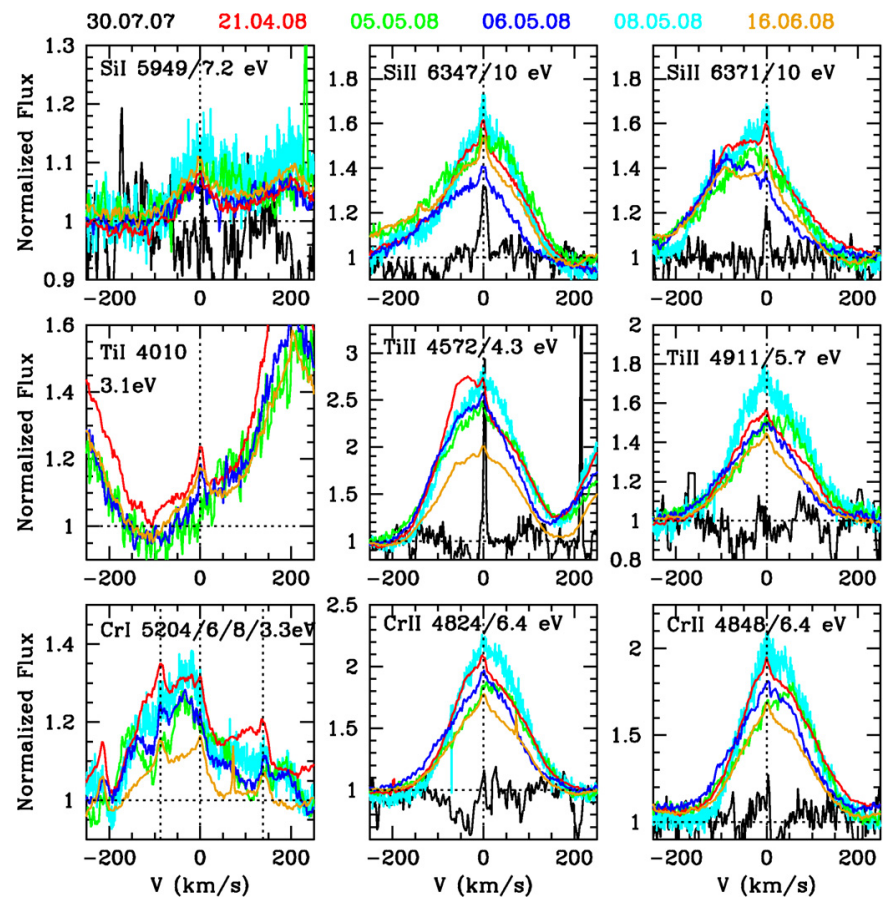

Fig. 10. Some of the Si I, Si II, Ti I, Ti II, Cr I, and Cr II lines observed in emission during the outburst phase. The zero velocity is marked by a dotted line. For comparison, the quiescence spectrum of 2007 July 30 is displayed at the wavelengths where there is enough $\mathrm{S} / \mathrm{N}$.

metallic lines strengthens in June as the accretion rate drops and the $\mathrm{BC}$ become weaker, which is probably a contrast effect. A Na I line at $7113 \AA\left(E_{k}=4.9 \mathrm{eV}\right)$ also seems to be present, in addition to the accretion-related $\mathrm{Na}$ I D. There is no clear detection of Mn I during the outburst phase, probably owing to blends with stronger lines. Towards the red part of the spectrum, some K I and C I lines are detected in emission, although they are strongly affected by telluric absorption (Fig. 11). All of these lines show the dynamical variations observed in the Fe I lines, with similar velocity shifts, except for the Ca I $4227 \AA$ A line, whose profile remains very stable. This line has a low $E_{k}(2.9 \mathrm{eV})$, which could suggest an origin in a distinct cooler region, but other lines with $E_{k} \sim 3-3.5 \mathrm{eV}$ display the typical dynamical pattern described above. The maximum observed $E_{k}$ varies for each species, being $E_{k} \leq 5 \mathrm{eV}$ for Ca I, $E_{k} \leq 3 \mathrm{eV}$ for Ti I, $E_{k} \leq 4.2 \mathrm{eV}$ for Co I, $E_{k} \leq 7.5 \mathrm{eV}$ for $\mathrm{Mg} \mathrm{I}$, $E_{k} \leq 4.2 \mathrm{eV}$ for K I, $E_{k} \leq 7.5 \mathrm{eV}$ for Si I, $E_{k} \leq 6 \mathrm{eV}$ for Ni I, $E_{k} \leq 3.7 \mathrm{eV}$ for Cr I, and $E_{k} \sim 8.9-9.1 \mathrm{eV}$ for the C I lines.

Fe II and Ti II are the most common ionized lines found in the spectra. They are usually very strong and follow the dynamical patterns observed in neutral lines, although their profiles are affected by stronger absorption features. The April 2008 observations, which are the closest to the outburst maximum, provide evidence of both blueshifted and redshifted absorption, which are signatures of wind and infall, respectively, while the data from June 16, 2008 is more consistent with infall alone (Figs. 12 and 10). This is indicative of a combined origin for the lines in the accretion column and the accretion-related wind. The $E_{k}$ of the ionized lines have typical energies of the upper level $\sim 5.5 \mathrm{eV}$ for Fe II and $\sim 4 \mathrm{eV}$ for Ti II. We also observe some $\mathrm{Si}$ II and $\mathrm{Cr}$ II lines (with $E_{k} \sim 10$ and $\sim 6.5 \mathrm{eV}$; Fig. 10). There is no evidence of any other ionized lines. The relatively higher excitation potentials suggest formation in a region with higher temperatures and/or lower electron densities than the places where the neutral lines arise, as we discuss later on. 

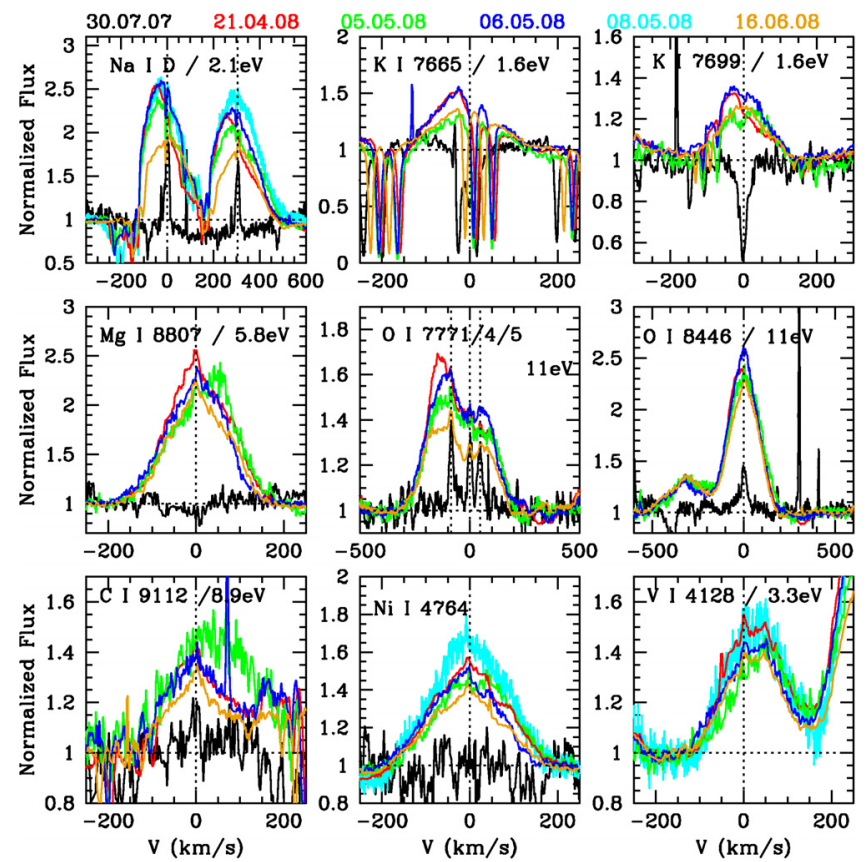

Fig. 11. Examples of other lines observed in emission during the outburst phase. The zero velocity is marked by a dotted line. For comparison, the quiescence spectrum of 2007 July 30 is displayed. The color code is as in Fig. 5.

Although the Ca II IR triplet and the Ca II $\mathrm{H}$ and $\mathrm{K}$ lines follow the accretion/wind pattern observed in the $\mathrm{H}$ I Balmer lines, they also contain a $\mathrm{NC}$ centered at zero velocity, as seen in the rest of the metallic lines (Fig. 7). In contrast, other Ca II lines (e.g. Ca II 8912 and $8927 \AA$ A) display the same dynamical imprint as the rest of metallic lines. Finally, we also observe some O I lines (7771/4/5 $\AA$ triplet and $8446 \AA$ line; Fig. 11). The O I triplet appears to be strongly blueshifted, as has been seen in stars with strong accretion and wind emission (Hamann \& Persson 1992b).

\section{Discussion}

\subsection{Accretion and winds}

The broad and asymmetric profiles of the emission lines observed in young CTTS (especially, those of the H I Balmer series) are usually interpreted in terms of magnetospheric accretion and winds (Königl 1991; Muzerolle et al. 1998, 2001; Appenzeller et al. 1986; Hamann \& Persson 1992b; Alencar \& Basri 2000). Other mechanisms capable of producing the observed features in CTTS, such as extended winds and jets alone and emission from low-temperature H I gas (Kurosawa et al. 2006; Whelan et al. 2004; Bary et al. 2008) can be ruled out in the case of EX Lupi by noting the redshifted absorption and the ratio of $\mathrm{Br} \gamma$ to $\mathrm{Pa} \beta$ observed in the infrared (IR), consistent with high temperature H I (Sipos et al. 2009; Kóspal et al. 2011). The accretion-related lines observed in the 2008 EX Lupi outburst have wings extending up to relatively large velocities $\left( \pm 400 \mathrm{~km} \mathrm{~s}^{-1}\right)$ compared to other CTTS and to simple magnetospheric accretion models. Higher temperatures in the accretion flow contribute to increasing the widths of $\mathrm{H} \alpha$, especially when combined with high accretion rates (Kurosawa et al. 2006). The inclusion of wind components combined with detailed infall models can produce profiles in good agreement with our observations (e.g. Lima et al. 2010). While $\mathrm{H} \alpha$ and $\mathrm{H} \beta$ can be interpreted in terms of accretion, wind, and infall, the profiles of the higher Balmer lines (which are more optically thin, and thus
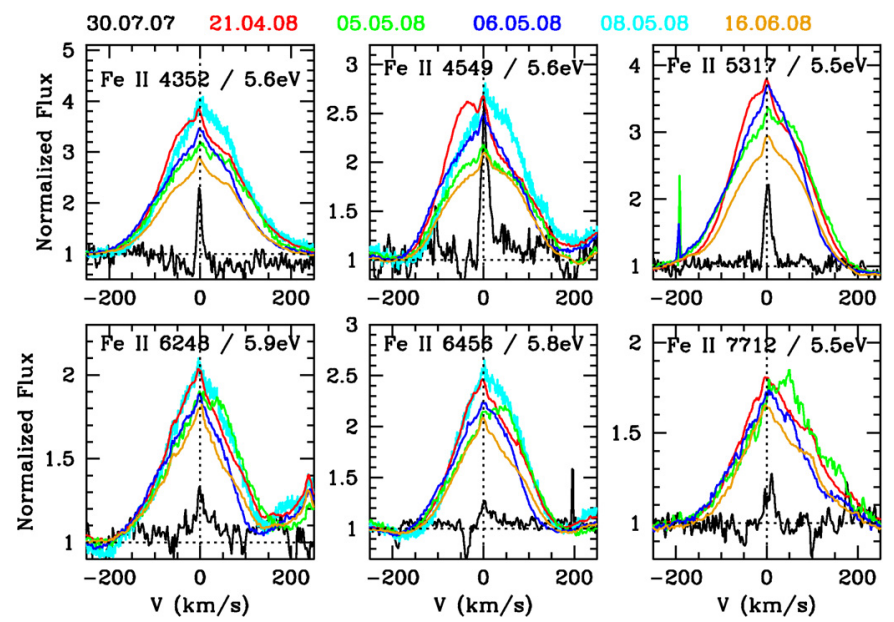

Fig. 12. Some of the Fe II lines seen in emission during the outburst phase. The zero velocity is marked by a dotted line. For comparison, the quiescence spectrum of 2007 July 30 is displayed. The color code is as in Fig. 5.

trace deeper/denser structures) reveal multi-component variable profiles. The stronger redshifted absorption present in the higher Balmer lines is indicative of an origin closer to the star. The weakening of the blueshifted absorption in the higher Balmer lines is expected if they originate in an optically thin wind (Hartmann et al. 1994).

It is remarkable that the velocities observed for the blueshifted and redshifted absorption components in the H I Balmer lines do not significantly differ in outburst and quiescence (although the blueshifted absorption component in quiescence is very weak). This suggests that the mechanisms and paths for both infall and outflow are not significantly different between outburst and quiescence, and only the amount of accreted and expelled matter varies. The post-outburst phase appears very similar to the pre-outburst, which implies that the system made a rapid recovery after the outburst, which is also consistent with the idea of stable accretion/outflow paths and mechanisms.

To make a simple estimate of the accretion rates during the pre-outburst, outburst, and post-outburst phases, we measured the $10 \%$ velocity wings of the $\mathrm{H} \alpha$ line $\left(\mathrm{H} \alpha_{10 \%}\right)$, which were on average 345,550 , and $300 \mathrm{~km} \mathrm{~s}^{-1}$, respectively. Following Natta et al. (2004), the $\mathrm{H} \alpha_{10 \%}$ correlates to the accretion rate (in $M_{\odot} / \mathrm{yr}$ ) according to

$\log (\mathrm{d} M / \mathrm{d} t)=-12.9( \pm 0.3)+9.7 \times 10^{-3}( \pm 0.7) \mathrm{H} \alpha_{10 \%}$.

We thus derive average accretion rates of $3 \times 10^{-10} M_{\odot} / \mathrm{yr}$, $3 \times 10^{-8} M_{\odot} / \mathrm{yr}$, and $1 \times 10^{-10} M_{\odot} / \mathrm{yr}$ during the pre-outburst, outburst, and post-outburst phase. The EW of the Li I $6708 \AA$ line, the only one clearly present in absorption during the outburst, agrees with these results, being $0.46,0.04$, and 0.6 during the pre-outburst, outburst, and post-outburst phases, respectively. These values and their variations are consistent with accretion-related veiling. During the outburst phase, the $\mathrm{H} \alpha_{10 \%}$ decreases from $>570 \mathrm{~km} \mathrm{~s}^{-1}$ to $\sim 540 \mathrm{~km} \mathrm{~s}^{-1}$ between April and June, which is consistent with a variation in the rate from $>4 \times 10^{-8} M_{\odot} /$ yr to $\sim 2 \times 10^{-8} M_{\odot} /$ yr. Although the spread in this relation is large, mostly due to line-of-sight effects, the observations suggest an increase in the accretion rate of about two orders of magnitude between quiescence and outburst, and a factor of a few higher accretion rate in the pre-outburst phase than in the post-outburst epoch. The accretion rates derived from Brackett $\gamma$ 
by Sipos et al. (2009) and Juhász et al. (2012) reveal an increase in $\dot{M}$ up to 3 orders of magnitude from quiescence to outburst (from $\sim 4 \times 10^{-10}$ to $2 \times 10^{-7} M_{\odot} / \mathrm{yr}$ ), which is consistent with our results, given that our $\mathrm{H} \alpha$ measurement in April is a lower limit $^{5}$. The line profile variations of $\mathrm{H} \alpha$ also agree with this picture (Fig. 6).

The presence of a wind appears in many TTS to be correlated to the accretion activity, with the mass loss due to wind being approximately $10 \%$ of the accretion rate (Calvet 1997). Although typical EXor outbursts are characterized by line profiles dominated by infall (inverse P Cygni profiles; Herbig 2007), the 2008 EX Lupi outburst presents strong evidence of a powerful wind, as noted by Aspin et al. (2010). The January spectra from Aspin et al. (2010) appear to detect a stronger wind absorption (below the continuum), which suggests that there was a higher accretion and outflow at earlier stages of the outburst, followed by a progressive decrease in the infall rate and wind absorption with time. The main evidence of a wind component comes from both the blueshifted absorption in $\mathrm{H} \alpha, \mathrm{H} \beta$, and the metallic lines, especially the ionized ones (Fe II, Ti II), observed in April 2008 and also reported in January 2008 by Aspin et al. (2010). The wind signatures are weaker in subsequently acquired spectra, being much weaker (or absent) in our May and June 2008 observations. The blueshifted absorptions in the ionized metallic lines correspond to smaller velocities than in the $\mathrm{H} I$ lines (up to $-100 \mathrm{~km} \mathrm{~s}^{-1}$ instead of $-200 \mathrm{~km} \mathrm{~s}^{-1}$ ), which may be a combined effect of optical thickness and element abundance in thewind.

The O I line at $8446 \AA$ is strong and relatively symmetric (Fig. 11). On the other hand, the O I 7771/4/5 $\AA$ triplet is consistent with the presence of a powerful wind and infall. In April 2008, the peak of the BC of the O I $7771 \AA$ line appeared both stronger than the $\mathrm{NC}$ and blueshifted by about $60 \mathrm{~km} \mathrm{~s}^{-1}$, and the line contained both blueshifted and redshifted absorption components. The blueshifted peak progressively transformed into a shoulder as the accretion rate decreased, probably owing to the wind becoming optically thinner. The infall-related redshifted absorption also decreased as the accretion rate becomes lower. Further evidence of blueshifted absorption is also found in the Na I D lines (Fig. 11).

This suggests that the 2008 outburst was extraordinary not only for EX Lupi, but also for the class of the EXors. The presence of a wind is a clear sign that the accretion rate exceeded the typical rates measured in EXor outbursts (Herbig et al. 2001), although the depth of the blueshifted absorption at its maximum is far from that typically seen in FUors (Hartmann \& Kenyon 1996). The presence of a powerful wind can also explain the transport of crystalline material to the outer disk, as suggested by Juhász et al. (2012) based on the variations in the mineralogy of the outer disk. In this case, the wind must extend beyond the relatively dense and warm regions observed by our optical spectra.

\subsection{Dynamics of the metallic line-emission region: stellar chromosphere and accretion column}

As we have mentioned before, in the quiescence phase, all the metallic lines are narrow $\left(F W H M \sim 10-20 \mathrm{~km} \mathrm{~s}^{-1}\right)$, and

\footnotetext{
5 Note that the accretion estimate in April is affected by the strong blueshifted absorption, so it can only be considered as a lower limit. To minimize the effect of the wind, if we assumed the line to be relatively symmetric, the resulting $\mathrm{H} \alpha_{10 \%}$ would be on the order of $630-650 \mathrm{~km} \mathrm{~s}^{-1}$, corresponding to an accretion rate of $2-3 \times$ $10^{-7} M_{\odot} / \mathrm{yr}$.
}

frequently appear superimposed on the corresponding (broader) photospheric absorption lines. The NCs of the metallic lines observed in the outburst phase strongly resemble the quiescence emission lines. In both cases, the lines appear centered on zero velocity and we can exclude velocity variations of over $3 \mathrm{~km} \mathrm{~s}^{-1}$ in individual lines, which is consistent with an origin very close to the stellar photosphere. The lines appear relatively symmetric and do not suffer significant profile variations, although their intensities change. The presence of weak metallic emission lines is relatively common among T Tauri stars (Hamann \& Persson 1992a), and they have also been observed in other EXors (Herbig 2008), although the case of EX Lupi in quiescence is remarkable because of the number and strength of the lines. The small velocities and the relatively low excitation potentials agree with the lines being produced in an active chromosphere (Hamann \& Persson 1992a). This picture is consistent with the available ultraviolet (UV) and X-ray observations (Grosso et al. 2010). The high X-ray absorption and UV variability, which do not correspond to the observed optical $A_{\mathrm{V}}$, suggest a large column density in thick, dense accretion flows, while hard-X ray variability reveals a very active chromosphere (Grosso et al. 2010).

The situation during the outburst phase is complex, given the strongly variable $\mathrm{BC}$ profiles in the metallic lines. Aspin et al. (2010) had already noticed variations in the line profile between their January and May 2008 observations. Our more-complete dataset reveals the interesting dynamics in the $\mathrm{BC}$ of the Fe I, Ca I, K I, Mg I, Ni I, and V I lines, which is also confirmed by the weaker Si I and Ti I lines present in the spectra. The BC of the neutral metallic lines suffers a day-to-day shift, with peak variations of up to $+50 \mathrm{~km} \mathrm{~s}^{-1}$. The shift is also seen in the ionized lines, although to a lesser extent, maybe due to the presence of stronger absorption features.

Rapid variations have been observed in accretion-related lines of very active TTS (e.g. RW Aur; Hartmann 1982; Alencar et al. 2005; DR Tau; Alencar et al. 2001), which are typically associated with non-steady accretion and outflow. More rarely, these variations are also found in the metallic lines of very active CTTS (e.g. SCrA; Appenzeller et al. 1986; Hamann \& Persson 1992a), although these variations tend to affect the intensity rather than the velocity components of the lines (e.g. Beristain et al. 1998). In Herbig Ae stars/UXors, velocity variations in the metallic absorption lines have been identified as infalling cold clumps of matter (Mora et al. 2002, 2004). In general, rapid variations in the emission lines are most accurately explained by global motions of large parcels of hot gas around the star. The dynamical variations in the metallic lines differ from the line profile variations owing to the variable accretion and wind observed in the H I Balmer series. This suggests that the H I lines and the metallic lines are produced (or dominated) by emission in physically distinct regions, although excitation conditions and optical thickness may also play a role. For instance, in regions with relatively low temperature $(<7000 \mathrm{~K})$, metallic lines are expected to dominate over $\mathrm{H}$ I emission, while the $\mathrm{H}$ I lines are produced in regions with higher temperatures (Beristain et al. 1998).

To determine the origin of the variability in the metallic lines, we analyzed in detail the magnitude of the shift for the lines that do not appear to be blended (Table 4). For each of these lines, we measured the central wavelength and FWHM using IRAF task splot by fitting a Gaussian profile to the lines. We find that all the lines vary consistently from day to day. We observe a clear variation of the central line velocity between approximately rest velocity and $+22 \mathrm{~km} \mathrm{~s}^{-1}$ (Table 5). The variations in the peak position of the $\mathrm{BC}$ are more extreme, and span up to $+50 \mathrm{~km} \mathrm{~s}^{-1}$. 
Table 5. Central line velocities.

\begin{tabular}{lccccc}
\hline \hline Elements & Apr. 21 & May 5 & May 6 & May 8 & June 16 \\
\hline Fe I, Ca I, Mg I & $1.7 \pm 2.0(20)$ & $22.2 \pm 2.3(20)$ & $-1.5 \pm 2.0(20)$ & $8.7 \pm 2.0(18)$ & $0.3 \pm 1.9(20)$ \\
Fe II, Si II, Ti II, Cr II & $5.0 \pm 2.3(29)$ & $16.4 \pm 3.2(29)$ & $-4.5 \pm 2.9(29)$ & $11.5 \pm 2.5(26)$ & $3.5 \pm 2.7(29)$ \\
All & $3.3 \pm 1.4(60)$ & $16.8 \pm 2.0(60)$ & $-3.5 \pm 1.6(60)$ & $10.2 \pm 1.6(48)$ & $2.7 \pm 1.5(60)$ \\
\hline
\end{tabular}

Notes. Velocities derived from the Gaussian fit of the lines in Table 4. For each date and type of lines, we give the average velocity with standard deviation and the number of points used in the calculation (in brackets). Only the lines that appear independent and are well-identified (listed in Table 4) have been taken into account in our calculations.

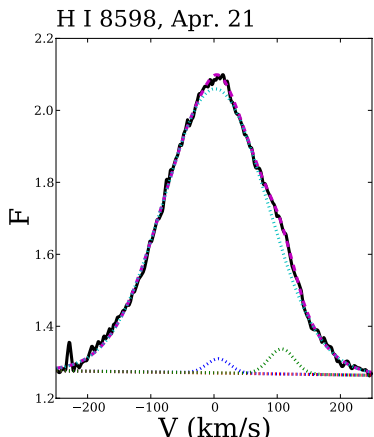

$\mathrm{V}(\mathrm{km} / \mathrm{s})$
H I 8598, May 05

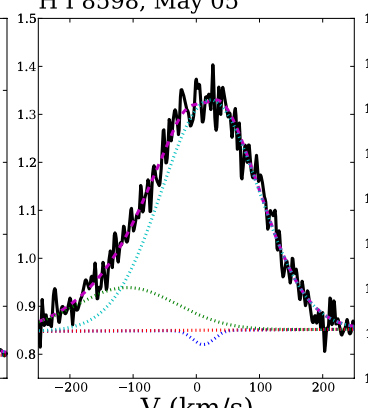

$\mathrm{V}(\mathrm{km} / \mathrm{s})$
H I 8598, May 06

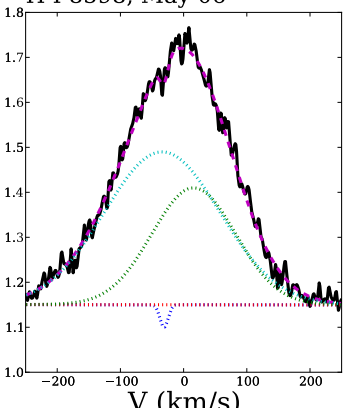

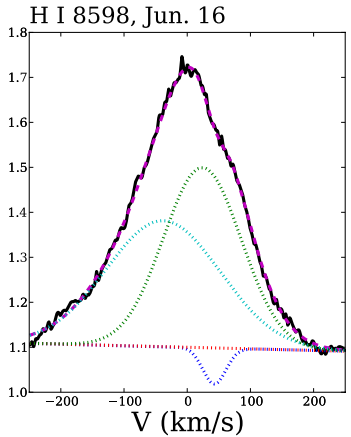

Fig. 13. H I $8598 \AA$ line: results of the fit with three Gaussian components for the different outburst epochs. The original data is shown as the bold line, individual Gaussians are marked by dotted lines, and the final fit is shown as a dashed line.
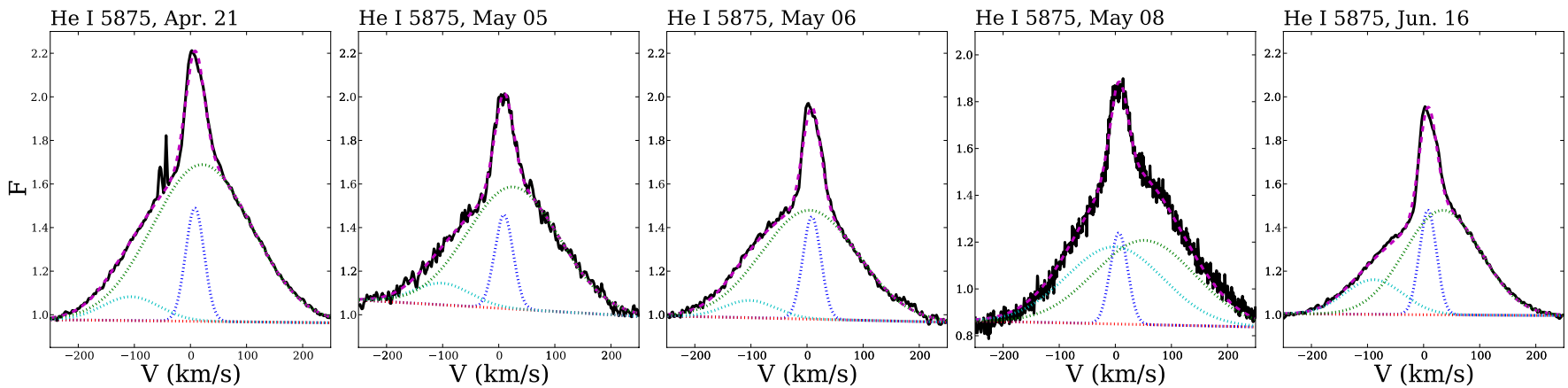

Fig. 14. He I $5875 \AA$ A line: results of the fit with three Gaussian components for the different outburst epochs. The original data is shown as the bold line, individual Gaussians are marked by dotted lines, and the final fit is shown as a dashed line.

To get a more detailed picture of the line structure, we performed a line fit with the IRAF task ngaussfit using three Gaussian components for a selected sample of lines thought to have different origins (see the Appendix for more details), including $\mathrm{H} \mathrm{I}$, He I, and a large number of non-blended metallic lines. We avoided the Balmer series since their profiles are highly complex and differ from all the other lines. Some of the best fits are displayed in Figs. 13 to 16, with more lines being discussed in the Appendix. This exercise reveals at once clear differences between $\mathrm{H} \mathrm{I}$, He I, and the metallic neutral/ionized lines. The most remarkable features are the lack of $\mathrm{NC}$ in the $\mathrm{H} \mathrm{I}$ lines, and the lack of dynamical signatures in both the H I and $\mathrm{He}$ I lines, which trace the hotter and denser parts of the flow/shock region. The profiles of H I and He I lines are very stable, compared to the metallic lines. The dynamical signatures observed in the neutral and ionized metallic lines are very similar and strongly correlated.

The three Gaussian fits confirm that the $\mathrm{NC}$ velocities are essentially constant in time (within our level of precision), with the He I NC being the broadest, followed by the NC of the ionized lines and finally, the NC of the neutral metallic lines. This is in good agreement with a relatively stable chromosphere where the lines with the higher excitation potentials are produced in hotter, more turbulent regions. Alternative explanations for an origin of narrow He I lines in the post-shock environment have also been proposed (Beristain et al. 2001), although we do not measure consistent redshifts in the He I lines. We also confirm that the $\mathrm{BC}$ of $\mathrm{H} \mathrm{I}$ and $\mathrm{He}$ I lines have very different profiles compared to the metallic lines, as expected if they have physically different origins within the accretion column. In general, the $\mathrm{H} \mathrm{I}$ and $\mathrm{He} \mathrm{I}$ lines are more symmetric and, although some redshifted and blueshifted components are observed, they never reach the velocity shifts observed in the metallic lines, suggesting that the dynamical changes observed in the latter occur in a cooler region.

The 2008 May 5, 6, and 8 observations of the neutral metallic lines can be very well-reproduced with a narrow (FWHM 10-20 ̊) Gaussian component for the NC, plus two broader Gaussians with variable center velocity and width. The strongly redshifted component in the 2008 May 5 observations of the neutral and ionized metallic lines becomes very evident with the three Gaussian models, and we confirm the velocity offset of 

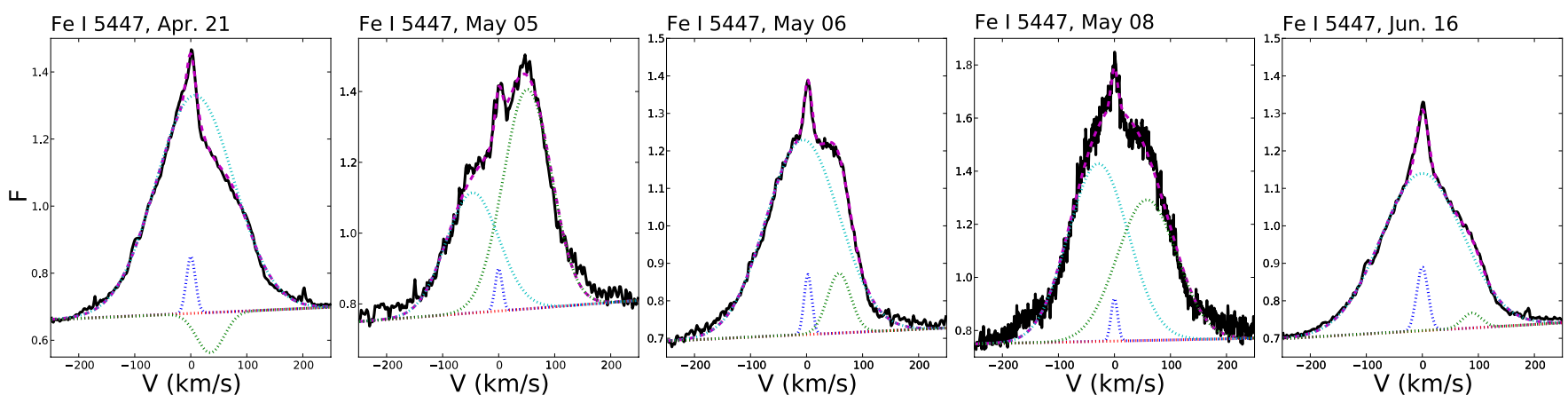

Fig. 15. Fe I $5447 \AA$ A line: results of the fit with three Gaussian components for the different outburst epochs. The original data is shown as the bold line, individual Gaussians are marked by dotted lines, and the final fit is shown as a dashed line.
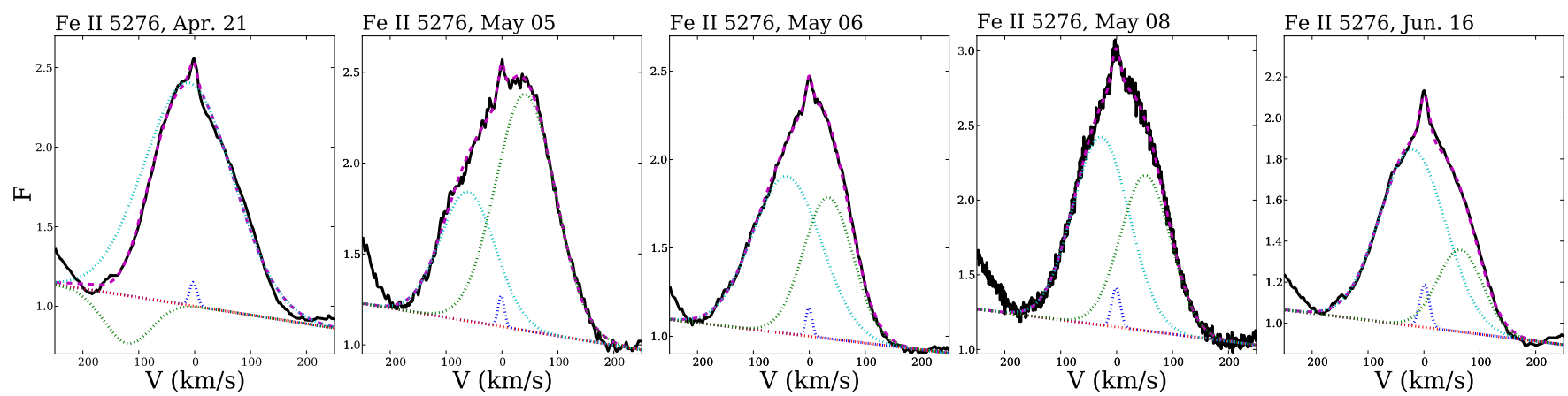

Fig. 16. Fe II $5276 \AA$ line: results of the fit with three Gaussian components for the different outburst epochs. The original data is shown as the bold line, individual Gaussians are marked by dotted lines, and the final fit is shown as a dashed line.

$\sim 50 \mathrm{~km} \mathrm{~s}^{-1}$. The velocity offset is maximal on 2008 May 5 , decreases by May 6 , and the line is clearly more symmetric by May 8. The corresponding observations of ionized metallic lines are also interpretable in the same terms, but the velocity offsets vary. In particular, although the maximum shift appears on May 5 for both neutral and metallic lines and has the same velocity in both groups, the ionized lines are much more symmetric on May 6 than the neutral lines, which is also indicative of a slightly different physical origin.

The observations of 2008 April 21 and June 16 are harder to interpret. While the $\mathrm{NC}$ remains stable, the $\mathrm{BC}$ profiles can be reproduced in several ways. The ionized lines can be well-described in terms of a broad emission component with blueshifted and/or redshifted absorption. There is a tendency for the April 21 data to be best fitted with a blueshifted absorption (indicative of wind), while a redshifted absorption most closely fits the June 16 data, although most spectra have signs of both components. The distinction is not so clear in the neutral metallic lines, which can be fitted with similar wind/infall components, but also with combinations of two Gaussian components in emission, although in these cases the shifts between the Gaussian components are less extreme than in May. Despite the more evident signatures of wind and infall in the ionized metallic lines, we are also unable to fully rule out the presence of two different Gaussian components in the line profiles of the ionized lines.

In summary, the rapid variations observed in May are indicative of a rapid dynamical change, probably caused by rotation plus infall of gas clumps, and the simple line-fitting confirms that the observed metallic lines are indeed consistent with a non-axisymmetric structure with several distinct components. The models of optical photometry and mid-IR interferometric observations during the outburst phase developed by
Juhász et al. (2012) proposed that a hot component dominated the spectral energy distribution (SED) at optical wavelengths, which could be assimilated to a large hot spot on the stellar surface, or a narrow ring of hot gas at the inner edge of the dusty disk. The net velocity shifts observed in the optical lines agree with the Keplerian velocity of material near the inner edge of the dusty disk (0.2-0.35 AU; Sipos et al. 2009), although the presence of redshifted absorption features is a clear sign of infall, so we have most likely a combination of rotation and infall. The rapid variations in the line profiles and velocities suggest that, rather than an axisymmetric hot, rotating disk (or ring), we observe a non-axisymmetric structure that spirals in onto the star from distances consistent with the inner disk rim. This agrees with the CO observations by Goto et al. (2011) and the near-IR data by Kóspal et al. (2011), which detected an asymmetric structure that could correspond to either an accretion column or matter flow spiraling in onto the star, although the $\mathrm{CO}$ emission is restricted to a cooler region than the source of the metallic lines. Rotation and infall of a non-axisymmetric, thick accretion flow would naturally shift the bulk velocity of the material as observed in the spectra. On the other hand, the fact that the broad component is always visible places limits on the possibility of occultation by the star, and suggests that either we view the system (or the accretion flow) from a high angle, or the structure, although not axisymmetric, is relatively extended in the azimuthal and/or vertical direction. This includes the possibility of having more than one accretion column, and is also consistent with the astrometric signal detected by Kóspal et al. (2011) for hydrogen emission. The parcels of gas traveling within the column would increase in temperature and velocity as they approached the star. A similar scenario, although with cold gas clumps instead of hot matter, can explain the transient absorption events seen in UXors 
and Herbig Ae stars (Mora et al. 2002, 2004), although our more scarce data does not allow us to define a rotation pattern.

\subsection{Physical conditions in the line-emitting region}

The relative intensities of the emission lines can be used to investigate the physical conditions (in particular of density and temperature) of the regions where the emission originates, and whether the emitting region can be considered to be in local thermodynamical equilibrium (LTE). The line ratios for many lines commonly seen in CTTS have been calibrated in terms of temperature and density, and can also provide information about saturation or collisional de-excitation. For other lines, the Saha equation can be applied to derive the physical properties of the emitting gas, assuming LTE. Here, we combine these methods to constrain the physical conditions in the line-emitting region.

The accretion-related emission lines in EX Lupi display typical CTTS behavior in both the quiescence and the outburst phases. In the $\mathrm{H}$ I Balmer series (especially, $\mathrm{H} \alpha$ and $\mathrm{H} \beta$ ), we observe the usual saturation pattern found in CTTS (e.g. Hamann \& Persson 1992a). The lower Balmer lines are easily saturated under the typical conditions in CTTS. The components of the Ca II IR triplet ${ }^{6}$ have relative intensities closer to unity than to the 1:9:5 relation expected from their transition coefficients, which is suggestive of formation in high density environments, as is also common in CTTS (Hamann \& Persson 1992a). This sets a lower limit to the electronic density of $10^{11} \mathrm{~cm}^{-3}$ for temperatures in the range 4000-10000 K (Hamann \& Persson 1992a). The Na I doublet usually appears to be close to saturation in CTTSs, and this is also consistent with what we observe in EX Lupi, although instead of the normal 2:1 ratio, we observe instead that the strength of the second component is about $\sim 80 \%$ of the first one. During the quiescence phase, the emission lines are rarely blended. The Mg I lines at $5167 \AA$, $5172 \AA$, and $5183 \AA$ show similar intensities in the pre- and post-outburst spectra, as expected from LTE. The O I triplet at 7771/4/5 $\AA$ also displays intensities consistent with the theoretical LTE values in quiescence. Similar estimates in outburst are highly uncertain owing to the uncertainty in the continuum levels and the presence of blends. We can also provide some initial constraints on the temperature by considering an upper limit of $10000 \mathrm{~K}$ (to ensure the presence of neutral hydrogen) and even $<7000 \mathrm{~K}$, applying the requirement that the metallic lines and the $\mathrm{H}$ I emission are produced in different places, as suggested by their different dynamics (Beristain et al. 1998). A lower limit to the temperature can be set as the temperature of an M0 star ( $3800 \mathrm{~K})$. Juhász et al. (2012) found that the optical SED in outburst is dominated by a continuum emission that can be fitted as black body emission of temperature $\sim 6500 \mathrm{~K}$, which is also a good indicator of the dominant temperatures in the line-emitting region during the outburst.

We can check both the temperature and density conditions of the emitting region by using the Saha equation (Mihalas 1978) for a two-component atom-ion pair. This provides only a limited approach: in the real case, we would have to include a large number of atoms and ions of different species and their levels, and consider that the LTE assumption may not be correct. Nevertheless, this simple exercise can provide some basic information on the order of magnitude of temperatures and densities required in the line formation region. The Saha equation

\footnotetext{
6 The $8542 \AA$ component is affected by the FEROS gap, but the intensity of the line during the outburst can be estimated from the observed peak, even if the line in full is not visible.
}

gives the relation between two successive states of ionization of a given species in LTE

$\frac{N_{j+1} n_{\mathrm{e}}}{N_{j}}=\left(\frac{2 \pi m k T}{h^{2}}\right)^{3 / 2} \frac{2 U_{j+1}(T)}{U_{j}(T)} \mathrm{e}^{-\chi_{I} / k T}$,

where, $N_{j+1}, N_{j}$, and $n_{\mathrm{e}}$ represent the number of atoms in the $j+1$ and $j$ ionization states and the electron number density, $T$ is the temperature, $m$ is the electron mass, $\chi_{I}$ is the ionization potential, and $U_{j+1}$ and $U_{j}$ are the partition functions for the $j+$ 1 and $j$ states. The level populations are calculated according to the Boltzmann distribution, and can thus be transformed into expected line intensity ratios to compare with the observed data. For our estimates, we used the data and Saha equation calculator provided by the NIST database, varying the electron density and temperature within reasonable limits and checking a single pair atom/ion at a time.

For the outburst, we first concentrated on the very abundant $\mathrm{Fe}$ I and Fe II lines, estimating the approximate ratios from line peaks. This is only valid if the line profiles are similar and we can assume that both Fe I and Fe II originate in the same region (we discuss the origin perhaps not being exactly the same later on). In general, for a given wavelength, Fe II lines are twice as strong as Fe I lines (Figs. 9 and 12). To obtain a typical 1:2 line ratio for Fe I:Fe II for a temperature of $6500 \mathrm{~K}$, we need an electronic density of around $n_{\mathrm{e}} \sim 5 \times 10^{12} \mathrm{~cm}^{-3}$. This value is consistent with the requirements for the saturation of the Ca II IR triplet. The line ratio is very sensitive to the electron density, so changes by a factor of few would result in temperatures from $5800 \mathrm{~K}$ (for $n_{\mathrm{e}} \sim 1 \times 10^{12} \mathrm{~cm}^{-3}$ ) up to $7000 \mathrm{~K}$ (for $n_{\mathrm{e}} \sim 1 \times 10^{13} \mathrm{~cm}^{-3}$ ). If we now checked the relative intensities of other neutral/ion pairs for the same density and temperature ranges, we would expect intensity ratios of $1-2$ orders of magnitude for Ti II relative to Ti I lines, of about a factor of five for Si II relative to Si I, and a factor of about two for $\mathrm{Cr}$ II relative to $\mathrm{Cr}$ I. This is roughly consistent with the observations (Fig. 10). In quiescence, this exercise becomes harder owing to the lower $\mathrm{S} / \mathrm{N}$ and the smaller number of observed lines. When we concentrate again on the Fe I/II lines, we observe similar or slightly larger Fe II:Fe I ratios (2-3). Since the stronger lines are those with lower excitation potentials, the temperature is expected to be lower than in the outburst phase, so the differences in the line ratios are probably caused by a lower electron density.

For the He I/He II lines, if we assumed the minimum electronic density $n_{\mathrm{e}} \sim 10^{11} \mathrm{~cm}^{-3}$ derived from the Ca II IR triplet, we would need temperatures in the $15000 \mathrm{~K}$ range to obtain a detectable emission of He II at $4686 \AA$. Since He II is observed in both outburst and quiescence, this is valid for all epochs. Higher densities would require even hotter temperatures, but too high electron densities would produce He I emission lines at longer wavelengths (which are not detected) comparable or stronger than the detected He II line. We would not expect any detectable He emission assuming the temperature ranges required for the metallic lines, but the line profiles clearly have different origins. The He I NC and the He II line are most likely produced in the transition zone between the chromosphere and the stellar corona, at high densities and temperatures. The He I BCs, on the other hand, probably originate in the hottest parts of the accretion flow. To produce the H I Balmer series in emission we typically need lower temperatures than for the He lines, and the differences in the profiles of $\mathrm{H}$ I and He I lines imply that they are formed in different places. We can explain why the Balmer lines do not show any evidence of the dynamical effects seen in the metal lines considering that the $\mathrm{H} \mathrm{I}$ emission of the metallic-emitting 
region is overwhelmed by the $\mathrm{H} \mathrm{I}$ emission from hotter environments closer to the star.

The NC and BC of the metallic lines in the outburst phase are produced in different environments, since the superposition of the physical locations of the $\mathrm{NC}$ and $\mathrm{BC}$ areas would result in the $\mathrm{BC}$ being the only visible one. Estimating the line ratios for the $\mathrm{NC}$ would require more accurate line models, given the difficulties in extracting the line from a profile-variable $\mathrm{BC}$ and an unknown continuum level. In principle, since all metallic lines have a $\mathrm{NC}+\mathrm{BC}$ structure, and since we do not see any $\mathrm{NC}$ standing alone (although in some very faint $\mathrm{Ti}$ I lines, the $\mathrm{BC}$ is undetected among the strong nearby lines and continuum), we would expect similar temperatures in the region responsible for the NC. A temperature of $\sim 6500 \mathrm{~K}$ would be consistent with a chromospheric origin. The enhanced Ti I NC emission could be reproduced assuming either slightly lower temperatures or higher electron densities. The NC was stronger in June, which is probably due to the decrease in the continuum and $\mathrm{BC}$ emission related to the lower accretion rates.

An independent density analysis can be derived from the accretion rate. If we assume that the line-emitting region we see in the outburst corresponds to the accretion flow, the measure of the accretion rates and line velocities can be used to derive a mass density in the proximities of the star that can be compared with the densities derived by other means. We consider an accretion rate of $2 \times 10^{-7} M_{\odot} / \mathrm{yr}$ and the velocities observed in the emitting lines, which have widths of $F W H M \sim 200-300 \mathrm{~km} \mathrm{~s}^{-1}$, depending on the line, $>300 \mathrm{~km} \mathrm{~s}^{-1}$ for H I Balmer lines, $\sim 230 \mathrm{~km} \mathrm{~s}^{-1}$ for Ca II lines, $\sim 180 \mathrm{~km} \mathrm{~s}^{-1}$ for typical metallic lines. We note that the line velocities are very similar to the free-fall velocities expected for magnetospheric infall from a $5 R_{*}$ distance (Gullbring et al. 1998), if we assume the stellar radius to be $R_{*}=1.6 R_{\odot}$ and a stellar mass of $0.6 M_{\odot}$ (Sipos et al. 2009). Assuming that the full accretion column lands onto the star, its cross-section can be considered as a fraction of the stellar surface, $f$, with $f<1$. The gas density in the accretion column can be estimated from the accretion rate, considering the velocity and the cross-section of the flow. Assuming the mean particle mass of interstellar matter, $\mu=1.36 \times 10^{-24} \mathrm{~g}$, we arrive at a number density of $\sim 1.3 / f \times 10^{12} \mathrm{~cm}^{-3}$. If the coverage fraction were on the order of $10 \%$ of the stellar surface, the density would thus be $\sim 10^{13} \mathrm{~cm}^{-3}$. For the relevant temperature ranges, we can assume that all metals are essentially ionized but that hydrogen and helium remain neutral, so that the electron density is very similar to the number density of metals, or about $0.1 \%$ of the total particle density, assuming a solar composition for the accretion flow. Nevertheless, this would result in an electron density two to three orders of magnitude below the estimate for a temperature $\sim 6500 \mathrm{~K}$, and one to two orders of magnitude below the requirement for saturation in the Ca II IR triplet. Therefore, the most plausible solution is that we have strong extra ionization, probably due to energetic radiation from the star, which ionizes up to a few percent of the $\mathrm{H}$ (see for instance Kwan \& Fischer 2011). Another solution would be to assume that the emitting region is enhanced in metals, but this would be hard to achieve if we identify the origin of the metallic emission with an accretion column, considering that the increased accretion event observed in 2008 resulted in a total mass accretion of about 50-70 Earth masses ${ }^{7}$.

\footnotetext{
7 Note that some degree of metal enrichment or depletion, for instance, caused by deviations in the standard dust-to-gas ratio, is possible due to grain growth, photoevaporation, and the potential formation of
}

To summarize, assuming reasonable densities $\left(n_{\mathrm{e}} \sim\right.$ $\left.10^{11}-10^{16} \mathrm{~cm}^{-3}\right)$ and temperatures $(T \sim 4000-10000 \mathrm{~K})$ for an accretion flow environment, we obtain a minimum temperature of $4500 \mathrm{~K}$ to attain the observed Fe II:Fe I line intensity ratio of $\sim 2: 1$ with the minimum density required to obtain similar fluxes in the Ca II IR triplet during the outburst phase. Choosing the value of $\sim 10000 \mathrm{~K}$, the typical temperature in the accretion shock of T Tauri stars (Hartigan et al. 1990; Gullbring et al. 1998), results in densities as high as $10^{16} \mathrm{~cm}^{-3}$ to ensure that there is a large enough Fe I fraction, but this temperature seems excessive for Ti I emission, unless we consider much higher densities, which are hard to attain with the measured accretion rates (at least, over large regions with a large velocity spread). A high temperature $(>7000 \mathrm{~K})$ is also inconsistent with the lack of variable dynamical signatures in the $\mathrm{H}$ I lines, relative to the metallic lines. The lower $E_{k}$ observed in the quiescence phase point to lower temperatures, so that the larger Fe II: Fe I ratio observed in quiescence would require lower electron densities.

As a last remark, we need to consider that the emitting region has most likely a non-uniform distribution of temperatures and densities. That the line profiles of neutral atoms and ions during the outburst phase are different, with the neutral atoms being more sensitive to the velocity variations in the accretion column and the ions displaying stronger signatures of wind and infall, suggests that they have slightly different origins, or that the predominant emission region is not the same in both cases. Therefore, the BC of the Fe II and other ionized lines are probably produced in a less dense region (for instance, the outer parts of the accretion column), while the bulk of the neutral emission arises from a denser region in the hot, non-axisymmetric accretion flow. Evaporation in the outer layers of the accretion flow could also explain why the wind-related blueshifted signatures are more pronounced in the ionized lines. A more detailed calculation of temperature and density would require complex models including the accreting star, its active chromosphere and hot spots, the non-axisymmetric accretion flow, and a disk wind component.

\subsection{Dynamics and physical conditions in the outburst phase}

The excitation potentials $\left(E_{k}\right)$ and transition probabilities (or Einstein coefficients, $A_{k i}$ ) of the lines can be roughly translated into temperatures and densities. Transitions with higher $E_{k}$ typically require a higher temperature, and those with lower Einstein coefficients (longer transition times) tend to originate in regions of lower density. As we have mentioned before, in quiescence the lines with lower excitation potentials $\left(E_{k} \sim 2-4 \mathrm{eV}\right)$ tend to be stronger than those with higher excitation potentials $\left(E_{k} \sim\right.$ 4-6 eV), but the situation is the opposite during the outburst phase: lines with lower excitation potentials tend to be weaker than those with higher excitation potentials. Lehmann et al. (1995) reported the inverse effect during the 1993-4 EX Lupi outburst, with the low excitation potential lines being stronger at outburst, which could be interpreted as cooling of the region where the lines are produced. In contrast, our observations suggests that the line-emitting zone was hotter and more extended in outburst than in quiescence, which could be explained by the increase in heating caused by the enhanced accretion.

We have thus explored the potential correlation of $E_{k}$ and $A_{k i}$ with the line properties, given by their central line velocity and FWHM, of neutral and ionized metallic lines observed during

protoplanets, which are all processes expected to occur to some degree in typical circumstellar disks. 
Table 6. Significance of the potential correlations between line dynamics, $E_{k}$, and $A_{k i}$

\begin{tabular}{|c|c|c|c|c|c|c|}
\hline Quantities & $R / p(1)$ & $R / p(2)$ & $R / p(3)$ & $R / p(4)$ & $R / p(5)$ & Comments \\
\hline Neutrals: $V$ vs. $E_{k}$ & $0.14 / 0.49$ & $-0.41 / 0.03$ & $-0.26 / 0.19$ & $0.07 / 0.76$ & $0.25 / 0.21$ & No correlation \\
\hline Ions: $V$ vs. $E_{k}$ & $-0.13 / 0.47$ & $-0.10 / 0.58$ & $-0.37 / 0.04$ & $-0.32 / 0.11$ & $-0.26 / 0.15$ & No correlation \\
\hline Neutrals: $F W H M$ vs. $E_{k}$ & $0.47 / 0.01$ & $0.53 / 0.004$ & $0.49 / 0.01$ & $0.48 / 0.02$ & $0.25 / 0.21$ & Correlation (except on 5) \\
\hline Ions: $F W H M$ vs. $E_{k}$ & $0.07 / 0.73$ & $0.06 / 0.74$ & $0.11 / 0.56$ & $0.25 / 0.22$ & $-0.06 / 0.75$ & No correlation \\
\hline Neutrals: $V$ vs. $A_{k i}$ & $-0.19 / 0.34$ & $-0.20 / 0.32$ & $-0.21 / 0.30$ & $0.09 / 0.70$ & $0.04 / 0.84$ & No correlation \\
\hline Ions: $V$ vs. $A_{k i}$ & $-0.39 / 0.03$ & $-0.55 / 0.001$ & $-0.36 / 0.05$ & $-0.21 / 0.30$ & $-0.30 / 0.11$ & Mild anticorrelation \\
\hline Neutrals: $F W H M$ vs. $A_{k i}$ & $0.07 / 0.74$ & $0.17 / 0.40$ & $0.06 / 0.78$ & $0.51 / 0.02$ & $-0.08 / 0.78$ & No correlation \\
\hline Ions: $F W H M$ vs. $A_{k i}$ & $0.56 / 0.001$ & $0.57 / 9 \mathrm{E}-4$ & $0.44 / 0.01$ & $0.43 / 0.03$ & $0.61 / 3 \mathrm{E}-4$ & Correlation \\
\hline
\end{tabular}

Notes. Potential correlations between the line dynamics (represented as center velocity $[V]$ and $F W H M$ of the BC of the metallic neutral and ionized lines) and physical conditions (represented by $E_{k}$ and $A_{k i}$ ) during the outburst phase. The results of a Spearman rank test are tabulated for each line type (ions/neutrals) and date, with $R$ being the correlation coefficient, and $p$ the false alarm probability. (1) 2008 April 21 ; (2) 2008 May 05; (3) 2008 May 06; (4) 2008 May 08; (5) 2008 June 16.

the outburst (Table 4). There is no evident correlation of $E_{k}$ with the central line velocity of the neutral and ion lines (Table 6). A Spearman test gives probabilities that the two quantities are uncorrelated of $4-60 \%$ for ions, and $3-75 \%$ for neutrals. It would seem that the correlation is sometimes significant, but that the correlation coefficients change sign over the different days indicates that both quantities are uncorrelated. On the other hand, there is a mild correlation of the FWHM of the neutral atoms with $E_{k}$ in all but the last observation, with positive correlation coefficients of around 0.5 , and probabilities of $0.4-2 \%$ that the two quantities are uncorrelated. This would be consistent with a larger FWHM being related to higher turbulence, and thus higher temperature regions, where the lines with higher $E_{k}$ can be excited. There is no evident correlation between FWHM and $E_{k}$ for ions. Regarding $A_{k i}$, there is a weak anticorrelation between the central line velocity of the ionized lines and $A_{k i}$, which would be consistent with the higher velocity components coming from the less dense regions, as expected in a wind scenario. The Spearman test indicates that the probability that these two quantities are uncorrelated are 3\%, 0.1\%, and 5\% for the first three observations, but the data are uncorrelated in the last two cases. At the same time, there is a clear correlation between the FWHM of ions and $A_{k i}$ (with Spearman probabilities in the range $0.03-1 \%$ that the two quantities are uncorrelated), which can be understood as a correlation between the higher temperatures (larger FWHM) and higher densities (large $A_{k i}$ ). No such correlations are observed for the neutral lines.

In summary, we find evidence that the lines with larger FWHM originate in hotter, denser environments, and that the ionized lines are dominated by less dense regions. This is consistent with formation in an infalling accretion column plus wind, but the general lack of strong trends points to a complex line-forming structure.

\section{Conclusions}

We have presented a collection of optical spectra of EX Lupi taken before, during, and after the 2008 outburst. The spectra contain a large number of emission lines. There is strong emission from the typical, accretion-related lines commonly observed in CTTS (H I Balmer and Paschen series, Ca II, He I). In addition, the spectra are rich in neutral metallic emission lines (Fe I, Ca I, Cr I, Ni I, Co I, Mg I, K I, C I), which appear superimposed on the photospheric absorption lines in the quiescence spectra, and an abundant range of metallic ionized lines (Fe II, Si II, Ti II, Cr II). The emission lines overwhelm any absorption feature during the outburst phase. We analyze these emission lines in order to determine the physical regions, conditions, and dynamics involved in the accretion processes, and how they changed during the phases of increased accretion in 2008.

1. The data are consistent with variable accretion being responsible for the observed outburst, with an increase of up to 2-3 orders of magnitude between quiescence and outburst. A wind (most likely an accretion-related inner disk wind) also developed in outburst, with an intensity that was correlated with the accretion rate that decreased as the outburst faded. The wind covers a large range of velocities, from -50 to $-200 \mathrm{~km} \mathrm{~s}^{-1}$. The presence of this wind agrees with the independent results of Goto et al. (2011) and Kóspál et al. (2011). During the quiescence phases, wind signatures were either weak or absent, and the main feature in the $\mathrm{H} \mathrm{I}$ Balmer lines was a redshifted absorption consistent with infall. The development of a strong wind in the outburst maximum is exceptional in the observed EX Lupi outbursts, and a sign of an accretion rate higher than in typical EXor outbursts, although still well below the values seen in FUors. Despite the strong wind, there is no evidence of any forbidden lines in emission related to shocks, so the presence of remnant envelopes or nearby molecular cloud material can be excluded.

2. The similarity between the pre- and post-outburst spectra is indicative of the rapid recovery of the system, suggesting that there was no dramatic change in the structure of the inner gaseous disk and accretion columns after the episode of increased accretion. The outburst is thus consistent with a strong variation in the accretion rate through remarkably stable accretion channels. The outburst represents thus an ideal environment to explore the way accretion proceeds in this object, and maybe also in similar EXors and even typical CTTS.

3. The observed metallic emission lines are narrow in quiescence and have a NC and a BC during the outburst. Their excitation potentials are consistent with the temperatures expected in the stellar chromosphere, hot spots, accretion columns, and shock region. Both the NC and the narrow emission lines observed in quiescence are consistent with an origin in an active chromosphere. The velocities observed in the $\mathrm{BC}$ during the outburst are too large for an origin near the stellar surface, and are thus most likely produced in an accretion column or a similarly extended and non-axisymmetric structure that heated up during the increased accretion episode. 


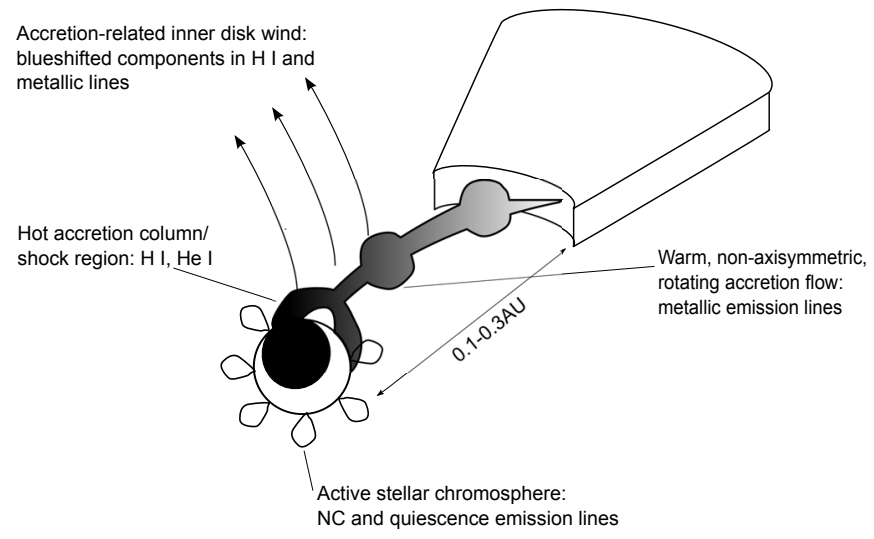

Fig. 17. A sketch of the proposed morphology of the accretion flow in EX Lupi to explain the optical spectroscopy data (not to scale). One or more clumpy accreting structures could be present in the system, and the geometry of the inner disk, accretion column(s), and accretionrelated wind is unconstrained.

4. We have observed dynamical changes in the BC of the metallic lines, that have timescales of days and are consistent with movement of large parcels of gas. The velocity variations were maximal on 2008 May 5, when the BC developed a secondary redshifted peak at $+50 \mathrm{~km} \mathrm{~s}^{-1}$, which became much fainter $24 \mathrm{~h}$ later, and absent after $72 \mathrm{~h}$. This is consistent with the dynamics of a hot and non-axisymmetric accretion flow or column(s), where independent parcels of accreting gas spiral-in in a clumpy accretion scenario (see a sketch of the system in Fig. 17). The picture of a rotating/infalling accretion flow is consistent with the observations of Aspin et al. (2010), the CO models of Goto et al. (2011; although note that $\mathrm{CO}$ traces a cooler region within the accretion flow/inner disk), and the interferometric observations of Juhász et al. (2012), which detected non-axisymmetric gaseous structures within the innermost disk. In addition, examples of the clumpy infall of gas parcels have been observed in UXor and Herbig Ae stars (Mora et al. 2002, 2004), although in these cases the infalling matter is colder and produces absorption features. The lack of dynamical signatures in the $\mathrm{H}$ I lines, compared to the metallic lines, can be explained if the $\mathrm{H}$ I emission is dominated by a distinct, hotter environment, compared to the metallic emission.

5. Juhász et al. (2012) found the optical component of the SED in outburst to be dominated by emission from a region with temperature $\sim 6500 \mathrm{~K}$. For such a temperature, if we assumed LTE, we would need a density of a few times $10^{12} \mathrm{~cm}^{-3}$ in the line emitting region in order to reproduce the observed line ratios for neutral/ion pairs (Fe I/Fe II, Si I/Si II, Ti I/Ti II, and $\mathrm{Cr}$ I/Cr II), which would also ensure the saturation of the Ca II IR triplet. Nevertheless, the line profiles suggest that the ionized lines may originate in a slightly different environment from the neutral lines. The stronger signatures of winds observed for ionized lines point to an origin in less dense areas dominated by the accretion-powered wind and/or by evaporation in the surface of the accretion column. In this case, the neutral region of the accretion flow could have a lower temperature and/or higher density. Extra sources of ionization (probably, the energetic radiation of the star) are required to achieve the desired electronic densities in the accretion column.
Acknowledgements. We thank the referee for a report that contributed to clarify this paper. A.S.-A. acknowledges support by the Deutsche Forschungsgemeinschaft, grant number SI 1486/1-1, and the Spanish "Ramón y Cajal" program, funded by the MICINN. We acknowledge the use of EX Lupi observations from the AAVSO International Database contributed by observers worldwide. We also thank N. Grosso for comments in the proof stage

\section{References}

Ábrahám, P., Juhász, A., Dullemond, C. P., et al. 2009, Nature, 459, 224 Alencar, S., \& Basri, G. 2000, AJ, 119, 1881

Alencar, S., Johns-Krull, C., \& Basri, G. 2001, AJ, 122, 3335

Alencar, S., Basri, G., Hartmann, L., \& Calvet, N. 2005, A\&A, 440, 595

Appenzeller, I., Jankovics, I., \& Jetter, R. 1986, A\&ASS, 64, 65

Aspin, C., Reipurth, B., Herczeg, G. J., \& Capak, P. 2010, ApJ, 719, L50

Bary, J. S., Matt, S. P., Skrutskie, M. F., et al. 2008, ApJ, 687, 376

Beristain, G., Edwards, S., \& Kwan, J. 1998, ApJ, 828, 852

Beristain, G., Edwards, S., \& Kwan, J. 2001, ApJ, 551, 1037

Calvet, N. 1997, Herbig-Haro Flows and the Birth of Stars, 182, 417

Edwards, S., Hartigan, P., Gandour, L., \& Andrulis, C. 1994, AJ, 108, 1056

Ferland, G. J., \& Persson, S. E. 1989, ApJ, 347, 656

Goto, M., Regály, Z., Dullemond, C. P., et al. 2011, ApJ, 728, 5

Gras-Velázquez, À., \& Ray, T. P. 2005, A\&A, 443, 541

Grosso, N., Hamaguchi, K., Kastner, J. H., Richmond, M. W., \& Weintraub, D. A. 2010, A\&A, 522, A56

Gullbring, E., Hartmann, L., Briceño, C., \& Calvet, N. 1998, ApJ, 492, 323

Hamann, F., \& Persson, S. E. 1992a, ApJS, 82, 247

Hamann, F., \& Persson, S. E. 1992b, ApJS, 82, 285

Hartigan, P., Hartmann, L., Kenyon, S., Strom, S., \& Skrutskie, M. 1990, ApJ, 354,25

Hartmann, L. 1982, ApJS, 48, 109

Hartmann, L., \& Kenyon, S., 1996, ARA\&A, 34, 207

Hartmann, L., Hewett, R., \& Calvet, N. 1994, ApJ, 426, 669

Herbig, G. H. 1950, PASP, 62, 211

Herbig, G. H. 1977, ApJ, 214, 747

Herbig, G. H. 1989, European Southern Observatory Conference and Workshop Proc., 33, 233

Herbig, G. H. 2007, AJ, 133, 2679

Herbig, G. H. 2008, AJ, 135, 637

Herbig, G. H., Aspin, C., Gilmore, A., Imhoff, C., \& Jones, A. 2001, PASP, 113, 1547

Herbig, G. H., Petrov, P., \& Duemmler, R. 2003, ApJ, 595, 384

Jones, A. 2008, Central Bureau Electronic Telegrams, 1217, 1

Juhász, A., Dullemond, C. P., van Boekel, R., et al. 2012, ApJ, 744, 118

Kaufer, A., Stahl, O., Tubbesing, S., et al. 2000, Proc. SPIE, 4008, 459

Königl, A. 1991, ApJ, 370, L39

Kóspál, A., Nemeth, P., Ábrahám, P., et al. 2008, Information Bulletin on Variable Stars, 5819, 1

Kóspál, Á., Ábrahám, P., Goto, M., et al. 2011, ApJ, 736, 72

Kurosawa, R., Harries, T. J., \& Symington, N. H. 2006, MNRAS, 370, 580

Kwan, J., \& Fischer, W. 2011, MNRAS, 411, 2383

Lehmann, T., Reipurth, B., \& Brandner, W. 1995, A\&A, 300, L9

Lima, G. H. R. A., Alencar, S. H. P., Calvet, N., Hartmann, L., \& Muzerolle, J. 2010, A\&A, 522, A104

Mason, E., Diaz, M., Williams, R. E., Preston, G., \& Bensby, T. 2010, A\&A, 516, A108

McLaughlin, D. B. 1946, AJ, 52, 109

Mora, A., Natta, A., Eiroa, C., et al. 2002, A\&A, 393, 259

Mora, A., Eiroa, C., Natta, A., et al. 2004, A\&A, 419, 225

Mihalas, D. 1978, Stellar Atmospheres (San Francisco: W.H. Freeman and Company)

Muzerolle, J., Hartmann, L., \& Calvet, N. 1998, AJ, 116, 455

Muzerolle, J., Calvet, N., \& Hartmann, L. 2001, ApJ, 550, 944

Nisini, B., Antoniucci, S., Giannini, T., \& Lorenzetti, D. 2005, A\&A, 429, 543

Parsamian, E. S., \& Mujica, R. 2004, Astrophysics, 47, 433; Translated from Astrofizika, 2004, 47, 507

Ralchenko, Yu., Kramida, A. E., Reader, J., \& NIST ASD Team 2010, NIST Atomic Spectra Database (ver. 4.0.1), 2011, National Institute of Standards and Technology, Gaithersburg, MD, http://physics.nist.gov/asd

Shu, F., Najita, J., Ostriker, E., et al. 1994, ApJ, 429, 781

Sipos, N., Ábrahám, P., Acosta-Pulido, J., et al. 2009, A\&A, 507, 881

Torres, A. V., \& Massey, P. 1987, ApJS, 65, 459

Whelan, E. T., Ray, T. P., \& Davis, C. J. 2004, A\&A, 417, 247

Pages 17 to 48 are available in the electronic edition of the journal at http: //www . aanda. org 


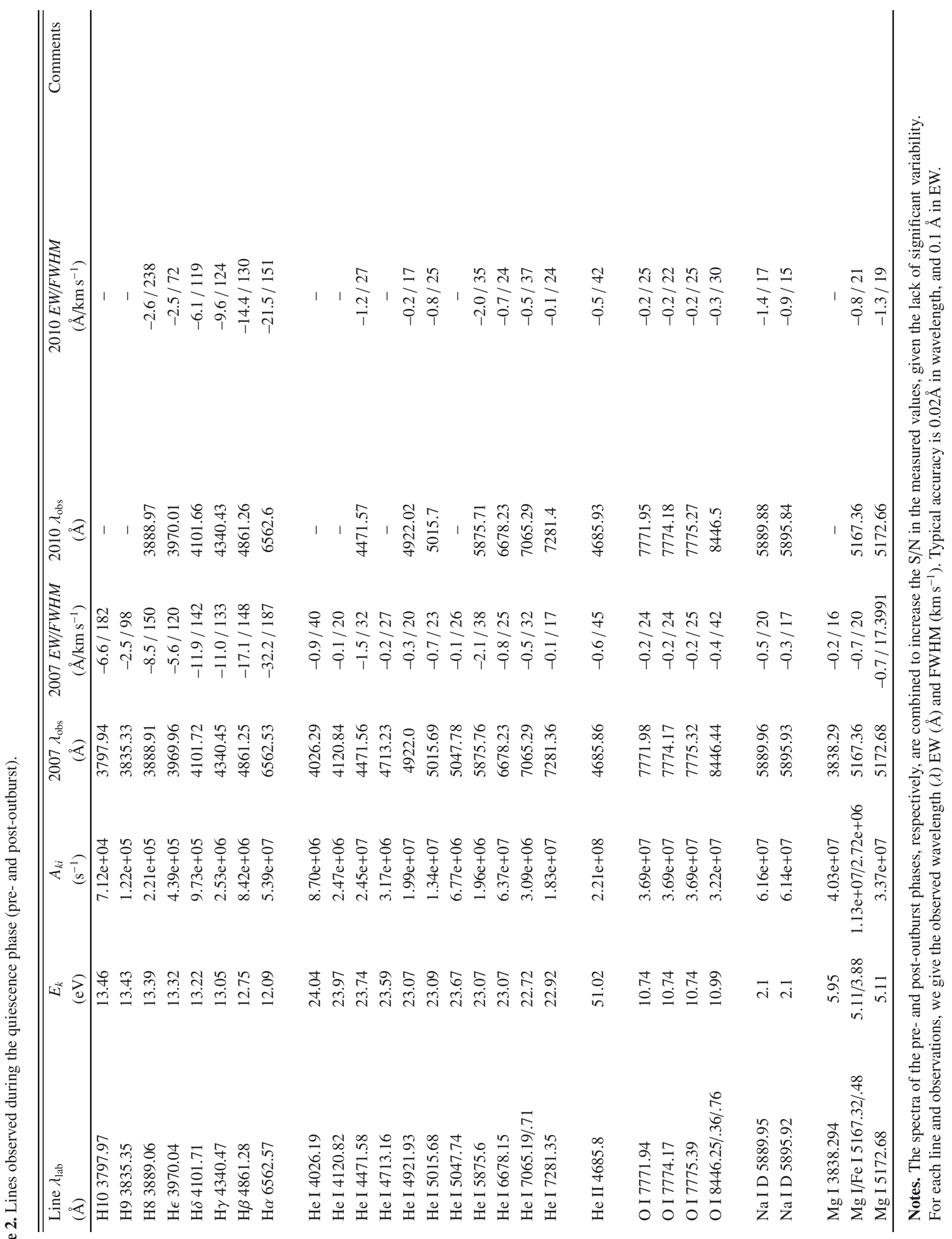




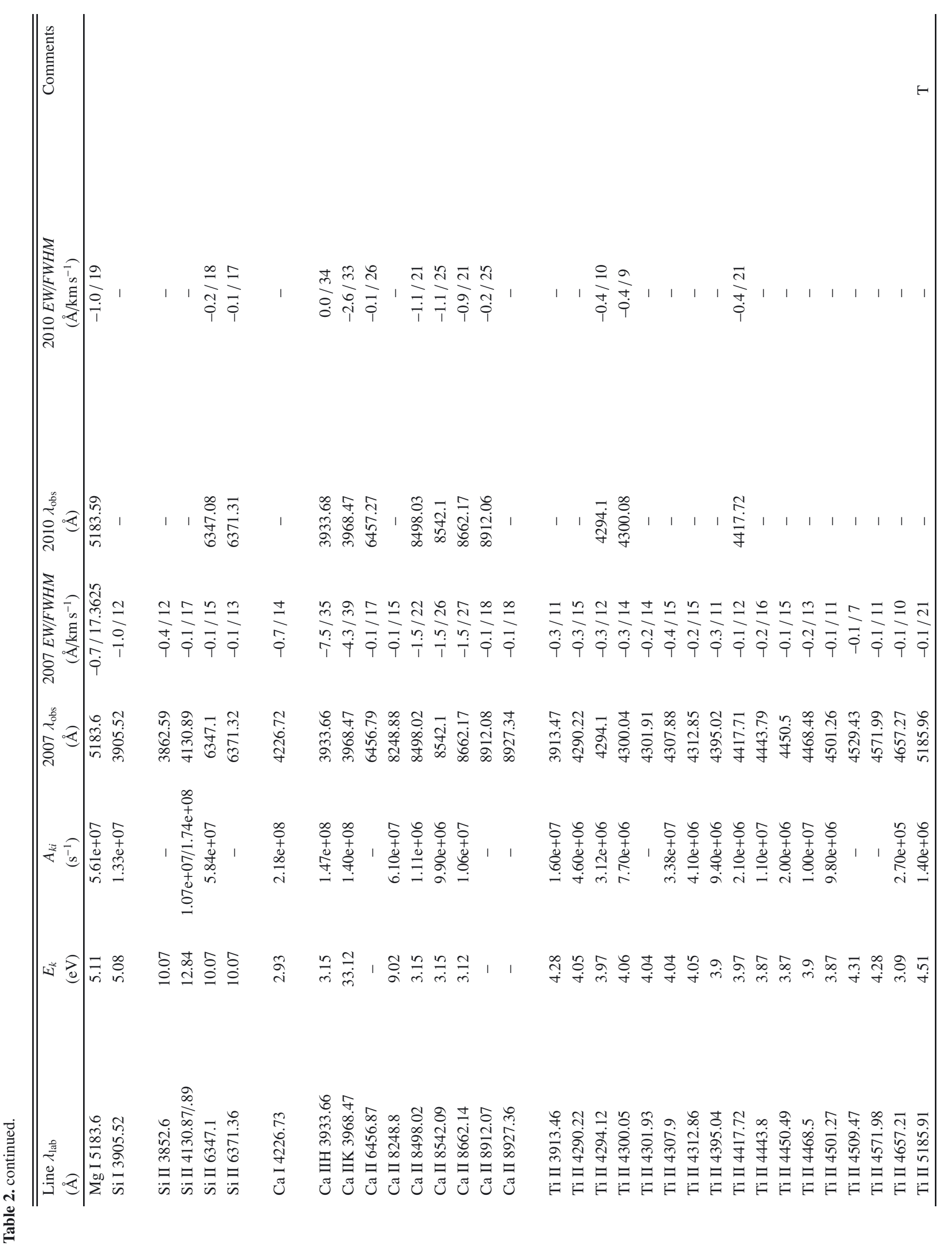




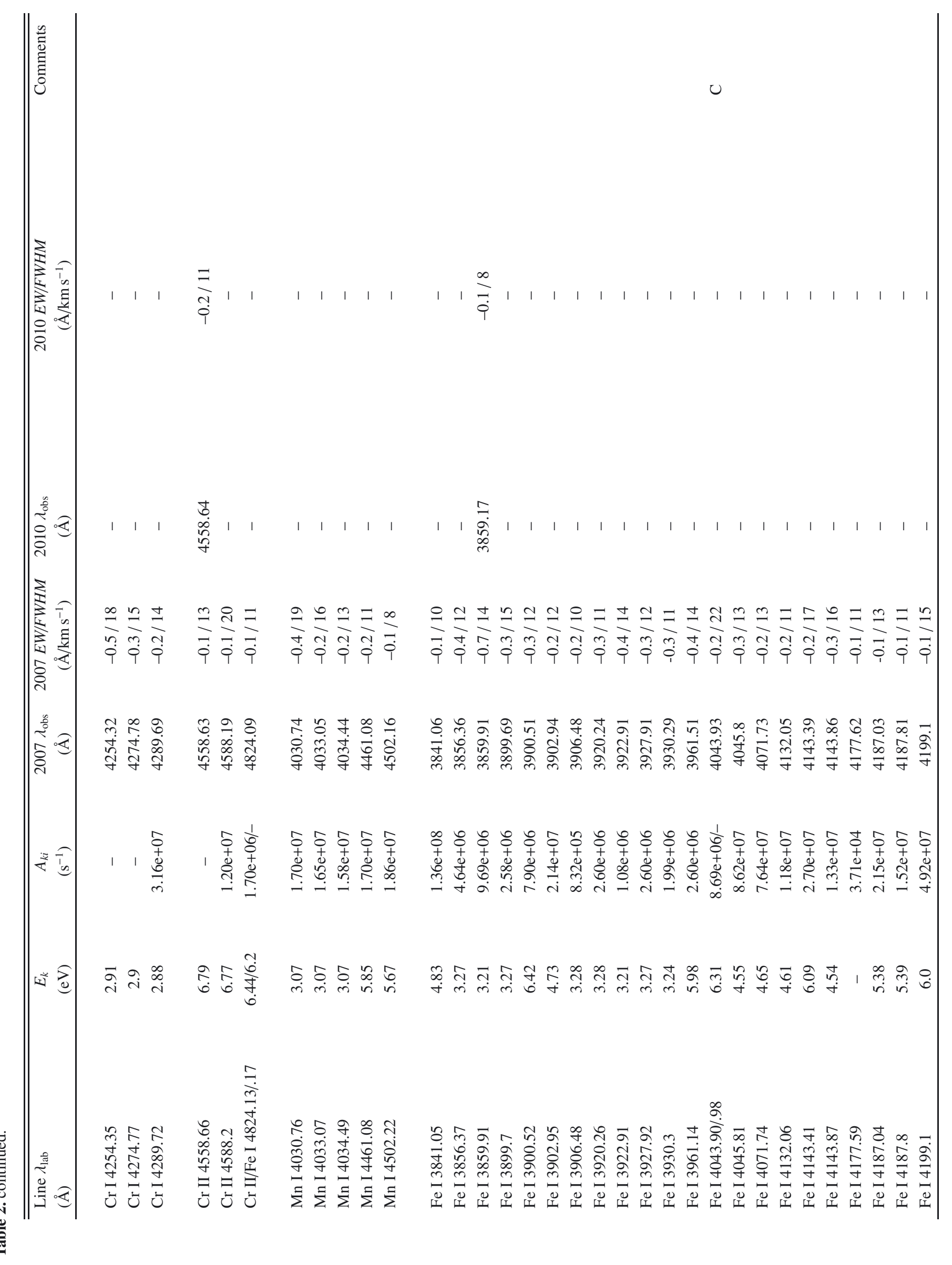




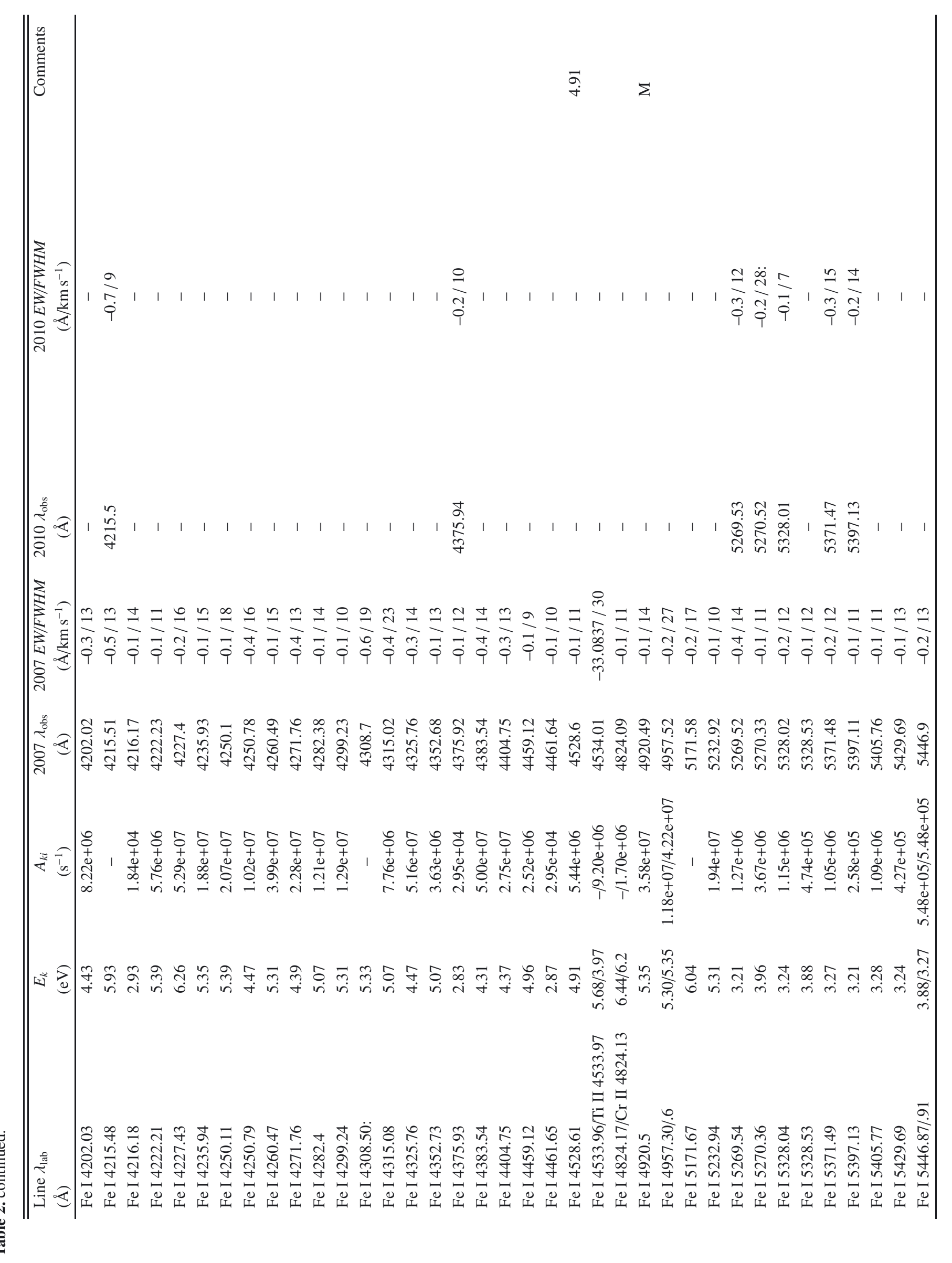




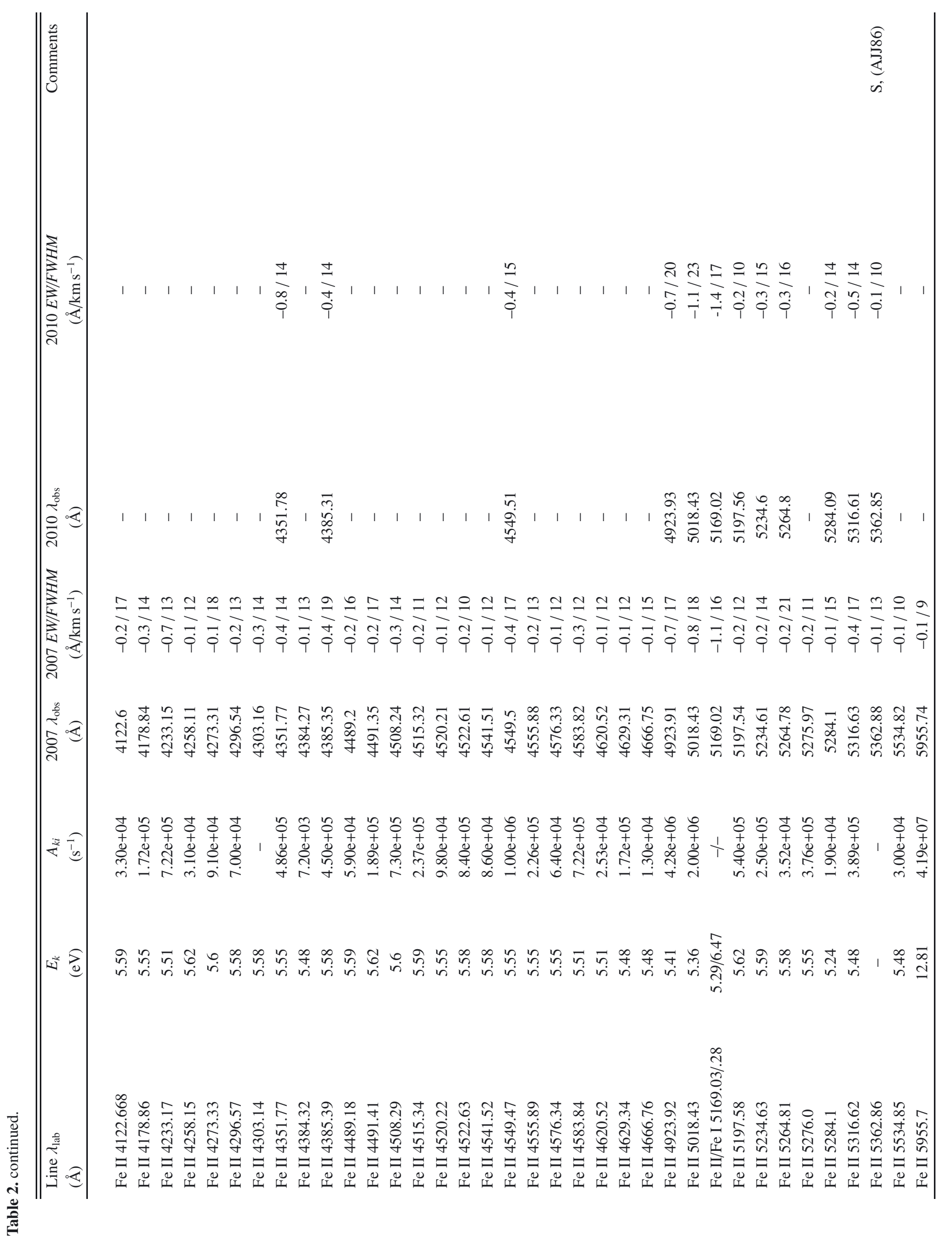


A\&A 544, A93 (2012)

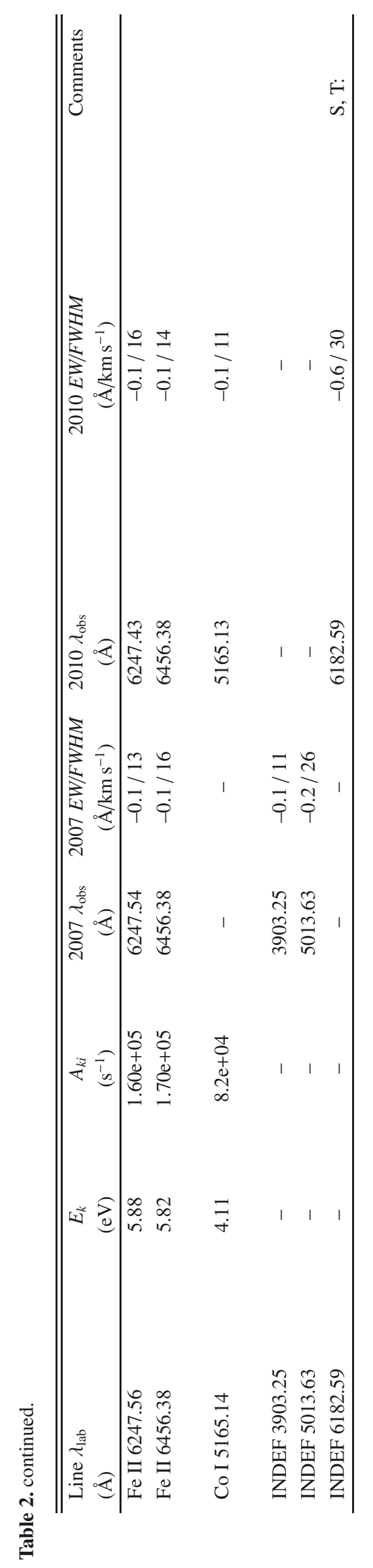

A93, page 22 of 48 
Table 3. Lines observed during the outburst phase, grouped according to element.

\begin{tabular}{|c|c|c|c|c|}
\hline $\begin{array}{l}\text { Line } \lambda_{\text {lab }} \\
(\AA)\end{array}$ & $\begin{array}{c}E_{k} \\
(\mathrm{eV})\end{array}$ & $\begin{array}{c}A_{k i} \\
\left(\mathrm{~s}^{-1}\right)\end{array}$ & $\begin{array}{l}\lambda_{\mathrm{obs}} \\
(\AA)\end{array}$ & Comments \\
\hline H I 3659.41 & 13.59 & $1.77 \mathrm{e}+02$ & 3659.41 & $\mathrm{BC}$ \\
\hline H I 3679.37 & 13.57 & $1.70 \mathrm{e}+03$ & 3679.38 & $\mathrm{BC}$ \\
\hline H I 3691.551 & 13.56 & $3.69 \mathrm{e}+03$ & 3691.53 & $\mathrm{BC}$ \\
\hline H I 3697.157 & 13.55 & $4.91 \mathrm{e}+03$ & 3697.1 & $\mathrm{BC}$ \\
\hline H I 3711.98 & 13.54 & $9.2 \mathrm{e}+03$ & 3712.21 & $\mathrm{BC}$ \\
\hline H I 3750.151 & 13.5 & $2.83 \mathrm{e}+04$ & 3750.24 & $\mathrm{BC}$ \\
\hline $\mathrm{H} \delta 4101.71$ & 13.22 & $9.73 e+05$ & 4101.0 & $\mathrm{BC}$ \\
\hline $\mathrm{H} \gamma 4340.47$ & 13.05 & $2.53 \mathrm{e}+06$ & 4339.8 & $\mathrm{BC}$ \\
\hline $\mathrm{H} \beta 4861.28$ & 12.75 & $1.72 \mathrm{e}+07$ & 4861.02 & $\mathrm{BC}$ \\
\hline Н $\alpha 6562.57$ & 12.09 & $5.39 \mathrm{e}+07$ & 6562.59 & $\mathrm{P}, \mathrm{BC}$ \\
\hline H I 8345.54 & - & - & 8345.59 & $\mathrm{BC}$ \\
\hline H I 8359.00 & - & - & 8359.20 & $\mathrm{BC}$ \\
\hline H I 8374.48 & 13.57 & - & 8374.75 & $\mathrm{BC}$ \\
\hline H I 8413.32 & 13.56 & $1.96 \mathrm{e}+03$ & 8413.31 & $\mathrm{BC}$ \\
\hline H I 8437.95 & 13.56 & $2.58 \mathrm{e}+03$ & 8437.76 & $\mathrm{BC}$ \\
\hline H I 8467.26 & 13.55 & $3.44 \mathrm{e}+03$ & 8468.18 & $\mathrm{BC}$ \\
\hline H I 8598.39 & 13.53 & $9.21 \mathrm{e}+03$ & 8598.51 & $\mathrm{P}, \mathrm{BC}$ \\
\hline H I 8750.46 & 13.54 & $2.02 \mathrm{e}+04$ & 8750.85 & $\mathrm{P}, \mathrm{BC}$ \\
\hline H I 9014.909 & 13.46 & $5.16 \mathrm{e}+04$ & 9014. & $\mathrm{~T}$ \\
\hline He I 3705.141 & 24.31 & $2.20 \mathrm{e}+06$ & 3705.22 & $\mathrm{~S} / \mathrm{N}, \mathrm{OA}$ \\
\hline He I $4143.761 / \mathrm{Fe}$ I 4143.868 & $24.21 / 4.55$ & $-/ 1.33 \mathrm{e}+07$ & 4143.83 & $\mathrm{pFe} I$ \\
\hline He I 4471.683 & $23.74 / 2.88$ & $1.37 \mathrm{e}+07$ & 4471.62 & B \\
\hline He I 4713.146 & 23.59 & - & 4713.23 & $\mathrm{~W}$ \\
\hline He I 5875.60 & 23.07 & $1.96 \mathrm{e}+06$ & 5875.77 & $\mathrm{P}$ \\
\hline He I 6678.151 & 23.07 & $6.37 \mathrm{e}+07$ & 6678.18 & $\mathrm{P}$ \\
\hline He I 7065.19 & 22.72 & - & 7065.29 & $\mathrm{P}$ \\
\hline He II 4685.80 & 51.02 & $2.21 \mathrm{e}+08$ & 4685.83 & $\mathrm{P}, \mathrm{NC}$ \\
\hline C I 8335.15 & 9.17 & $3.51 \mathrm{e}+07$ & 8335.09 & $\mathrm{~T}$ \\
\hline C I 9062.49 & 8.85 & $9.48 \mathrm{e}+06$ & 9061.79 & $\mathrm{~T}$ \\
\hline C I 9078.28 & 8.85 & $7.07 \mathrm{e}+06$ & 9078.21 & $\mathrm{~T}$ \\
\hline C I 9088.51 & 8.85 & $3.0 \mathrm{e}+07$ & 9088.77 & $\mathrm{~T}$ \\
\hline C I 9094.83 & 8.85 & $2.28 \mathrm{e}+07$ & 9094.9 & $\mathrm{~T}$ \\
\hline C I 9111.8 & 8.85 & $1.35 \mathrm{e}+07$ & 9111.48 & Wi \\
\hline O I 7771.94 & 10.74 & $3.69 \mathrm{e}+07$ & 7771.95 & \\
\hline O I 7774.17 & 10.74 & $3.69 \mathrm{e}+07$ & 7774.19 & \\
\hline O I 7775.39 & 10.74 & $3.69 \mathrm{e}+07$ & 7775.43 & \\
\hline O I 8446.25 & 10.99 & $3.22 \mathrm{e}+07$ & 8446.17 & \\
\hline
\end{tabular}

Notes. Lines classified as "INDEF" could not be found within the available line databases. The comments include "W" (weak line), "S" (strong line), "Wer" (line gets weaker with time), "Ser" (line gets stronger with time), "NC" (only the NC is clearly detected), "BC" (only the BC is detected), "M" (the line appears as a shoulder, merged with another stronger line), "B" (the line is a blend of several lines that cannot be disentangled), "S/N" (low S/N), "P" (the line has a clean profile, not being surrounded by other stronger lines), "Wi" (the profile shows blueshifted absorption consistent with an origin in a wind-like component), "Wi-I" (the line profile displays evidence of wind that turned into infall at later stages of the outburst), "Br" (remarkably broad line), "T" (line is contaminated by telluric absorption), "OA" (only observed in April), "WA" (weak in April), "SA" (strong in April), "WJ" (became weak in June), "SJ" (became stronger in June), "NJ" (not observed in June), "MJ" (line merged to a stronger one in June), "OJ" (only observed in June), "OQ" (only observed in quiescence), "p" (most probable classification, in the case of lines with two potential identifications, e.g. pFeI means that the line is probably the Fe I one, from the two listed), lines marked with: have an uncertain classification. Lines named INDEF could not be identified within the NIST database. In some cases, there is a nearby line (listed in the comments), but the offset in wavelength is much larger than typically observed. Excitation potentials and transition probabilities not listed in the NIST database are left blank. Some lines not listed in the NIST database but classified by Appenzeller et al. (1986) are identified with the comment AJJ86. 
Table 3. continued.

\begin{tabular}{|c|c|c|c|c|}
\hline $\begin{array}{l}\text { Line } \lambda_{\text {lab }} \\
(\AA)\end{array}$ & $\begin{array}{c}E_{k} \\
(\mathrm{eV})\end{array}$ & $\begin{array}{c}A_{k i} \\
\left(\mathrm{~s}^{-1}\right)\end{array}$ & $\begin{array}{l}\lambda_{\text {obs }} \\
(\AA) \\
\end{array}$ & Comments \\
\hline $\mathrm{Na}$ I 5889.95 & 2.1 & $6.16 \mathrm{e}+07$ & 5889.27 & \\
\hline Na I 5895.92 & 2.1 & $6.16 \mathrm{e}+07$ & 5895.41 & \\
\hline $\mathrm{Na}$ I 7113.04 & 4.93 & $4.43 e+04$ & 7113.02 & $\mathrm{~T}$ \\
\hline Na I 8194.79/K I 8191.24 & $3.62 / 4.18$ & $8.57 \mathrm{e}+06 /-$ & 0.0 & $\mathrm{~T}$ \\
\hline Na I 8183.256 & 3.62 & $4.29 \mathrm{e}+07$ & 0.0 & $\mathrm{~T}$ \\
\hline Al I 3961.52 & 3.14 & $9.8 \mathrm{e}+07$ & 3961.51 & $\mathrm{P}$ \\
\hline Al I 3944.006 & 3.14 & $4.9 \mathrm{e}+07$ & 3943.98 & \\
\hline Mg I 3807.41 & 7.6 & - & 3707.49 & \\
\hline Mg I 3831.680/Ni I 3831.69 & $7.58 / 3.66$ & $-/ 1.50 \mathrm{e}+06$ & 3831.73 & \\
\hline Mg I 3832.30 & 5.95 & $6.74 \mathrm{e}+07$ & 3832.23 & \\
\hline Mg I 4057.505 & 7.4 & $1.02 \mathrm{e}+07$ & 4057.47 & $\mathrm{~B}$ \\
\hline Mg I 4167.271/Mn I 4167.218 & $7.32 / 7.65$ & $1.38 \mathrm{e}+07 / 1.9 \mathrm{e}+06$ & 4167.26 & \\
\hline Mg I 5167.322 & 5.11 & $1.13 \mathrm{e}+07$ & 5167.35 & Ser \\
\hline Mg I 5172.684 & 5.11 & $3.37 \mathrm{e}+07$ & 5172.63 & \\
\hline Mg I 5183.604 & 5.11 & $5.61 \mathrm{e}+07$ & 5183.58 & \\
\hline Mg I 5528.405 & 6.59 & $1.39 \mathrm{e}+07$ & 5528.37 & $\mathrm{P}$ \\
\hline Mg I 5711.088 & 6.52 & $3.86 \mathrm{e}+06$ & 5711.07 & \\
\hline Mg I 7387.685 & 7.43 & $1.74 \mathrm{e}+06$ & 7387.56 & $\mathrm{~B}$ \\
\hline Mg I 7810.413 & 5.93 & - & 7810.36 & $\mathrm{~B}, \mathrm{~T}$ \\
\hline Mg I 8213.034 & 7.26 & $4.38 \mathrm{e}+06$ & 8213.68 & $\mathrm{~T}$ \\
\hline Mg I 8346.106/.119/.12 & 7.43 & $1.79 \mathrm{e}+05 /-$ & 8346.2 & $\mathrm{~B}$ \\
\hline Mg I 8736.006/.020/.021 & 7.36 & $2.86 \mathrm{e}+05 /-$ & 8735.94 & B: \\
\hline Mg I 8806.756 & 5.75 & $1.27 \mathrm{e}+07$ & 8806.72 & $\mathrm{P}$ \\
\hline Si I 4755.276 & 7.53 & $7.50 \mathrm{e}+05$ & 4755.3 & $\mathrm{~B}, \mathrm{~W}$ \\
\hline Si I 4792.324 & 7.54 & $1.70 \mathrm{e}+06$ & 4792.32 & $\mathrm{~B}, \mathrm{~W}$ \\
\hline Si I 5006.061/Fe I .128 & $7.56 / 5.31$ & $-/-$ & 5006.1 & B \\
\hline Si I 5772.145 & 7.23 & $3.56 \mathrm{e}+06$ & 5772.08 & W \\
\hline Si I 5797.859 & 7.09 & $2.53 \mathrm{e}+05$ & 5797.75 & W \\
\hline Si I 5948.545 & 7.17 & $2.2 \mathrm{e}+06$ & 5948.48 & \\
\hline Si I 6155.134 & - & - & 6155.15 & W \\
\hline Si I 7405.774 & - & - & 7405.73 & \\
\hline Si I 7409.082/Ni I 7409.35 & $-1-$ & $-1-$ & 7409.25 & \\
\hline Si I 7742.71/Fe I 7742.704 & $5.34 \mathrm{e}+06 / 6.59$ & $-/-$ & 7742.67 & \\
\hline Si I 7932.349 & 7.53 & $5.13 \mathrm{e}+06$ & 7932.4 & \\
\hline Si I 7944.001 & 7.54 & $5.75 e+06$ & 7944.02 & \\
\hline Si I 8073.03 & 7.81 & $1.80 \mathrm{e}+06$ & 8073.02 & \\
\hline Si I 8556.78 & - & - & 8556.64 & near Ca II \\
\hline Si I 8728.011 & - & - & 8727.94 & $\mathrm{~T}$ \\
\hline Si II 3862.60 & 10.07 & $3.91 \mathrm{e}+07$ & 3862.56 & WJ \\
\hline Si II 4130.87 & 12.84 & $1.07 \mathrm{e}+07$ & 0.0 & OQ \\
\hline Si II 5477.702 & 14.79 & $4.30 \mathrm{e}+05$ & 5477.78 & \\
\hline Si II 5957.56 & 12.15 & $5.60 \mathrm{e}+07$ & 5957.57 & WJ, B \\
\hline Si II 6347.10 & 10.07 & $5.84 \mathrm{e}+07$ & 6347.08 & Wi-I, P \\
\hline Si II 6371.36 & 10.07 & $6.80 \mathrm{e}+07$ & 6371.34 & Wi-I, P \\
\hline K I 4744.345 & 4.22 & $9.8 \mathrm{e}+04$ & 4744.38 & W \\
\hline K I 7664.90 & 1.62 & $3.80 \mathrm{e}+07$ & 7663.86 & $\mathrm{~T}$ \\
\hline K I 7698.96 & 1.61 & $3.75 \mathrm{e}+07$ & 7698.5 & $\mathrm{~T}$ \\
\hline
\end{tabular}


Table 3. continued.

\begin{tabular}{|c|c|c|c|c|}
\hline $\begin{array}{l}\text { Line } \lambda_{\text {lab }} \\
(\AA)\end{array}$ & $\begin{array}{c}E_{k} \\
(\mathrm{eV})\end{array}$ & $\begin{array}{c}A_{k i} \\
\left(\mathrm{~s}^{-1}\right)\end{array}$ & $\begin{array}{l}\lambda_{\mathrm{obs}} \\
(\AA)\end{array}$ & Comments \\
\hline K I 8194.24 & 4.18 & $3.10 \mathrm{e}+03$ & 8194.29 & $\mathrm{~T}$ \\
\hline Ca I 4108.526 & 5.73 & $9.00 \mathrm{e}+07$ & 4108.65 & M, SA \\
\hline Ca I 4226.73 & 2.93 & $2.18 \mathrm{e}+08$ & 4226.72 & \\
\hline Ca I 4302.53 & 4.78 & $1.36 \mathrm{e}+08$ & 4302.62 & $\mathrm{~B}$ \\
\hline Ca I 4434.96 & 4.68 & $6.70 \mathrm{e}+07$ & 4435.04 & \\
\hline Ca I 4454.779 & 4.68 & $8.70 \mathrm{e}+07$ & 4454.73 & \\
\hline Ca I 5041.620/Fe I 5041.756 & $5.17 / 3.94$ & $-/ 2.35 \mathrm{e}+05$ & 5041.69 & $\mathrm{pFe} I$ \\
\hline Ca I 5349.47 & - & - & 5349.47 & $\mathrm{C}$ \\
\hline Ca I 5512.98 & 5.18 & $1.10 \mathrm{e}+08$ & 5512.99 & $\mathrm{~B}$ \\
\hline Ca I 5581.97 & 4.74 & $6.0 \mathrm{e}+06$ & 5581.87 & \\
\hline Ca I 5594.47 & 4.74 & $3.8 \mathrm{e}+07$ & 5594.45 & \\
\hline Ca I 5598.48 & 4.74 & $4.30 \mathrm{e}+07$ & 5598.34 & \\
\hline Ca I 5601.29 & 4.74 & $8.6 e+06$ & 5601.28 & \\
\hline Ca I 5602.85 & 4.74 & $1.4 \mathrm{e}+07$ & 5602.86 & \\
\hline Ca I 5857.45 & 5.05 & $6.60 \mathrm{e}+07$ & 5857.44 & \\
\hline Ca I 6102.72 & 3.91 & $9.6 e+06$ & 6102.69 & \\
\hline Ca I 6122.22 & 3.91 & $2.87 \mathrm{e}+07$ & 6122.18 & $\mathrm{P}$ \\
\hline Ca I 6161.29 & 4.53 & $3.3 e+06$ & 6161.31 & \\
\hline Ca I 6162.17 & 3.91 & $4.77 \mathrm{e}+07$ & 6162.14 & \\
\hline Ca I 6166.44 & 4.53 & $2.20 \mathrm{e}+07$ & 6166.42 & $\mathrm{~W}$ \\
\hline Ca I 6169.06 & 4.53 & $1.7 \mathrm{e}+07$ & 6169.05 & \\
\hline Ca I 6169.560/Fe I 6169.597 & $4.53 / 6.74$ & $-/-$ & 6169.53 & $\mathrm{pCa} I$ \\
\hline Ca I 6439.07 & 4.45 & $5.3 e+07$ & 6439.04 & \\
\hline Ca I 6449.81 & 4.44 & $9.0 \mathrm{e}+06$ & 6449.79 & \\
\hline Ca I 6462.57 & 4.44 & $4.7 \mathrm{e}+07$ & 6462.62 & \\
\hline Ca I 6471.66 & 4.44 & $5.9 e+06$ & 6471.69 & \\
\hline Ca I 6493.78 & 4.43 & $4.40 \mathrm{e}+07$ & 6493.64 & \\
\hline Ca I 6717.69 & 4.55 & $1.2 \mathrm{e}+07$ & 6717.66 & $\mathrm{P}$ \\
\hline Ca IIH 3933.66 & 3.15 & $1.47 \mathrm{e}+08$ & 3934.01 & \\
\hline Ca IIK 3968.469 & 3.12 & $1.40 \mathrm{e}+08$ & 3967.92 & \\
\hline Ca II 5001.48 & 9.98 & $2.00 \mathrm{e}+07$ & 5001.45 & \\
\hline Ca II 8201.72 & 9.01 & $5.10 \mathrm{e}+07$ & 8201.69 & \\
\hline Ca II 8248.80 & 9.02 & $6.10 \mathrm{e}+07$ & 8248.84 & \\
\hline Ca II 8254.73 & 9.02 & $1.0 \mathrm{e}+07$ & 8254.69 & \\
\hline Ca II 8498.02 & 3.15 & $1.11 \mathrm{e}+06$ & 8497.99 & $\mathrm{P}$ \\
\hline Ca II 8542.09 & 3.13 & $9.90 \mathrm{e}+06$ & 8542.87 & \\
\hline Ca II 8662.14 & 3.12 & $1.06 \mathrm{e}+07$ & 8661.73 & $\mathrm{P}$ \\
\hline Ca II 8912.07 & - & - & 8912.04 & \\
\hline Ca II 8927.36 & - & - & 8927.33 & \\
\hline Sc II 4314.08 & 3.49 & $4.1 \mathrm{e}+08$ & 4314.06 & \\
\hline Ti I 4009.66/Fe I 4009.54 & $3.11 / 6.30$ & $1.21 \mathrm{e}+06 /-$ & 4009.65 & $\mathrm{~S}, \mathrm{NC}$ \\
\hline Ti I 4681.92 & 2.7 & $2.35 \mathrm{e}+06$ & 4681.9 & $\mathrm{~T}$ \\
\hline Ti I 4783.30/Mn I 4783.42 & $3.41 / 4.89$ & $6.20 \mathrm{e}+04 / 4.0 \mathrm{e}+07$ & 4783.34 & \\
\hline Ti I 5038.399 & 3.89 & $3.87 \mathrm{e}+07$ & 5038.43 & $\mathrm{OA}$ \\
\hline Ti I 5210.39 & 2.43 & $3.57 \mathrm{e}+06$ & 5210.21 & $\mathrm{~S} / \mathrm{N}$ \\
\hline Ti I 6258.10: & 3.42 & $8.36 \mathrm{e}+06$ & 6258.14 & no Ti I $6258.71 ?$ \\
\hline
\end{tabular}


Table 3. continued.

\begin{tabular}{|c|c|c|c|c|}
\hline $\begin{array}{l}\text { Line } \lambda_{\text {lab }} \\
(\AA)\end{array}$ & $\begin{array}{c}E_{k} \\
(\mathrm{eV})\end{array}$ & $\begin{array}{c}A_{k i} \\
\left(\mathrm{~s}^{-1}\right)\end{array}$ & $\begin{array}{l}\lambda_{\mathrm{obs}} \\
(\AA) \\
\end{array}$ & Comments \\
\hline Ti II 3913.46 & 4.28 & $1.6 \mathrm{e}+07$ & 3913.44 & \\
\hline Ti II 4028.34 & 4.97 & $5.1 \mathrm{e}+06$ & 4028.31 & \\
\hline Ti II 4053.834 & 4.95 & $4.20 \mathrm{e}+06$ & 4053.8 & \\
\hline Ti II 4161.535 & 4.06 & $2.10 \mathrm{e}+05$ & 4161.51 & Wi \\
\hline Ti II 4287.872 & 3.97 & $5.80 \mathrm{e}+05$ & 4287.9 & \\
\hline Ti II 4290.23 & 4.05 & $4.5 \mathrm{e}+07$ & 4290.2 & \\
\hline Ti II 4294.12 & 3.97 & $4.7 e+06$ & 4294.08 & $\mathrm{~S}, \mathrm{Wi}$ \\
\hline Ti II 4300.05 & 4.06 & $7.70 \mathrm{e}+06$ & 4300.02 & \\
\hline Ti II 4301.93 & 4.04 & $6.2 \mathrm{e}+06$ & 4301.92 & \\
\hline Ti II 4307.90 & 4.04 & $4.6 e+06$ & 4307.85 & P, Wi-I \\
\hline Ti II 4312.87 & 4.05 & $4.1 \mathrm{e}+06$ & 4312.86 & \\
\hline Ti II 4314.975 & 4.03 & $1.30 \mathrm{e}+07$ & 4314.94 & \\
\hline Ti II 4320.96 & 4.03 & $2.40 \mathrm{e}+06$ & 4320.72 & $\mathrm{~S}, \mathrm{P}, \mathrm{Wi}$ \\
\hline Ti II 4330.695 & 4.04 & $8.10 \mathrm{e}+05$ & 4330.72 & $\mathrm{P}$ \\
\hline Ti II 4367.659 & 5.43 & $1.90 \mathrm{e}+06$ & 4367.62 & B \\
\hline Ti II 4394.051 & 4.04 & $2.20 \mathrm{e}+06$ & 4394.19 & B \\
\hline Ti II 4395.04 & 3.9 & $2.9 \mathrm{e}+05$ & 4395.0 & \\
\hline Ti II 4399.772 & 4.05 & $3.10 \mathrm{e}+06$ & 4399.76 & \\
\hline Ti II 4407.678 & 4.03 & $5.80 \mathrm{e}+05$ & 4407.7 & \\
\hline Ti II 4417.72 & 3.97 & $2.10 \mathrm{e}+06$ & 4417.68 & M \\
\hline Ti II 4450.49 & 3.87 & $2.0 \mathrm{e}+06$ & 4450.44 & \\
\hline Ti II 4464.45 & 3.94 & $7.00 \mathrm{e}+05$ & 4464.43 & \\
\hline Ti II 4468.50 & 3.9 & $1.0 \mathrm{e}+07$ & 4468.47 & Wi-I \\
\hline Ti II 4501.27 & 3.86 & $9.8 \mathrm{e}+06$ & 4501.26 & Wi-I, S \\
\hline Ti II 4529.474 & 4.31 & $3.00 \mathrm{e}+05$ & 4529.45 & \\
\hline Ti II 4544.028 & 3.97 & $2.10 \mathrm{e}+05$ & 4543.98 & \\
\hline Ti II 4563.77 & 3.94 & $2.1 \mathrm{e}+07$ & 4563.74 & $\mathrm{P}$ \\
\hline Ti II 4571.98 & 4.28 & $1.2 \mathrm{e}+07$ & 4571.94 & P, Wi-I \\
\hline Ti II 4583.409 & 3.87 & $1.00 \mathrm{e}+05$ & 4583.35 & \\
\hline Ti II 4629.279 & 3.86 & $2.20 \mathrm{e}+05$ & 4629.31 & $\mathrm{P}$ \\
\hline Ti II 4708.665 & 3.87 & $3.10 \mathrm{e}+05$ & 4708.68 & \\
\hline Ti II 4779.985 & 4.64 & $6.20 \mathrm{e}+06$ & 4779.91 & \\
\hline Ti II 4798.521 & 3.66 & $1.80 \mathrm{e}+05$ & 4798.46 & $\mathrm{P}$ \\
\hline Ti II 4805.10 & 4.64 & $1.1 \mathrm{e}+07$ & 4805.03 & $\mathrm{P}$ \\
\hline Ti II 4911.193 & 5.65 & $3.2 \mathrm{e}+07$ & 4911.16 & $\mathrm{P}$ \\
\hline Ti II 5072.281 & 5.57 & $7.7 \mathrm{e}+06$ & 5072.36 & $\mathrm{~B}$ \\
\hline Ti II 5129.15 & 4.31 & $1.0 \mathrm{e}+06$ & 5129.02 & $\mathrm{~S}, \mathrm{P}$ \\
\hline Ti II 5188.70 & 3.97 & $2.5 \mathrm{e}+06$ & 5188.67 & \\
\hline Ti II 5226.56 & 3.94 & $3.1 \mathrm{e}+06$ & 5226.53 & $\mathrm{P}$ \\
\hline Ti II 5336.81 & 3.9 & $5.8 \mathrm{e}+05$ & 5336.76 & \\
\hline Ti II 5381.015 & 3.87 & $3.20 \mathrm{e}+05$ & 5380.98 & \\
\hline Ti II 6606.95 & 3.94 & $6.20 \mathrm{e}+04$ & 6606.94 & $\mathrm{~B}$ \\
\hline Ti II 7214.72 & 4.31 & $2.30 \mathrm{e}+05$ & 7214.73 & \\
\hline V I 4032.846 & 3.07 & $2.9 \mathrm{e}+05$ & 4032.86 & \\
\hline V I 4128.07 & 3.28 & $7.70 \mathrm{e}+07$ & 4128.11 & \\
\hline V I 5192.97/Ti I 5192.98 & $4.7 / 2.41$ & $4.0 \mathrm{e}+07 / 3.49 \mathrm{e}+06$ & 5192.93 & $\mathrm{~B}$ \\
\hline V I 6324.66 & - & - & 6324.51 & $\mathrm{NJ}$ \\
\hline Cr I 3850.04 & - & - & 3850.08 & $\mathrm{~B}$ \\
\hline Cr I 3902.920/Fe I 3902.946 & $4.16 / 4.73$ & $3.5 e+06 / 2.14 \mathrm{e}+07$ & 3902.94 & $\mathrm{~W}$ \\
\hline
\end{tabular}


Table 3. continued.

\begin{tabular}{|c|c|c|c|c|}
\hline $\begin{array}{l}\text { Line } \lambda_{\text {lab }} \\
(\AA)\end{array}$ & $\begin{array}{c}E_{k} \\
(\mathrm{eV})\end{array}$ & $\begin{array}{c}A_{k i} \\
\left(\mathrm{~s}^{-1}\right)\end{array}$ & $\begin{array}{l}\lambda_{\mathrm{obs}} \\
(\AA)\end{array}$ & Comments \\
\hline Cr I 4025.01 & - & - & 4024.91 & B \\
\hline Cr I 4077.09 & - & - & 4077.16 & \\
\hline Cr I 4077.68 & - & - & 4077.7 & \\
\hline Cr I 4127.30 & - & - & 4127.3 & \\
\hline Cr I 4254.35 & 2.91 & $3.15 \mathrm{e}+07$ & 4254.29 & B \\
\hline Cr I 4289.72 & 2.89 & $3.16 \mathrm{e}+07$ & 4289.72 & \\
\hline Cr I 4359.630/.617 & 3.83 & $5.4 \mathrm{e}+06 / 5.40 \mathrm{e}+06$ & 4359.64 & \\
\hline Cr I 4384.98 & 3.86 & $2.7 \mathrm{e}+06$ & 4384.98 & \\
\hline Cr I 4399.812 & 5.83 & $9.80 \mathrm{e}+06$ & 4399.99 & B \\
\hline Cr I 4496.86 & 3.7 & $3.3 e+06$ & 4496.85 & \\
\hline Cr I 4580.06 & 3.65 & $2.4 \mathrm{e}+06$ & 4580.03 & \\
\hline Cr I 4600.75 & 3.7 & $2.5 \mathrm{e}+06$ & 4600.74 & \\
\hline Cr I 4626.19 & 3.65 & $5.0 \mathrm{e}+06$ & 4626.21 & \\
\hline Cr I 4646.17 & 3.7 & $8.7 e+06$ & 4646.16 & \\
\hline Cr I 4651.28 & 3.65 & $3.6 e+06$ & 4651.27 & \\
\hline Cr I 4652.16 & 3.67 & $5.8 \mathrm{e}+06$ & 4652.12 & \\
\hline Cr I 4698.62 & - & - & 4698.65 & \\
\hline Cr I 4829.38 & - & - & 4829.38 & \\
\hline Cr I 4903.24 & 5.07 & $7.4 \mathrm{e}+06$ & 4903.24 & Wi \\
\hline Cr I $4942.500 / \mathrm{Fe}$ I 4942.459 & $3.45 / 6.73$ & $-/ 9.67 e+05$ & 4942.47 & $\mathrm{~W}$ \\
\hline Cr I 5204.52 & 3.32 & $5.09 \mathrm{e}+07$ & 5204.53 & \\
\hline Cr I 5206.04 & 3.32 & $5.14 \mathrm{e}+07$ & 5206.03 & \\
\hline Cr I 5208.44 & 3.32 & $5.06 \mathrm{e}+07$ & 5208.43 & \\
\hline Cr I 5247.56 & 3.32 & $1.9 \mathrm{e}+06$ & 5247.53 & \\
\hline Cr I 5254.92 & - & - & 5254.9 & \\
\hline Cr I 5296.69 & 3.32 & $3.1 \mathrm{e}+06$ & 5296.72 & B \\
\hline Cr I 5298.27 & 3.32 & $3.3 e+06$ & 5298.25 & \\
\hline Cr I 5345.81 & 3.32 & $4.9 \mathrm{e}+06$ & 5345.78 & \\
\hline Cr I 5348.32 & 3.32 & $1.7 \mathrm{e}+06$ & 5348.31 & \\
\hline Cr II 4012.47 & - & - & 4012.53 & \\
\hline Cr II 4242.45 & - & - & 4242.33 & $\mathrm{~B}, \mathrm{P},(\mathrm{AJJ} 86)$ \\
\hline Cr II 4558.66 & 6.79 & $8.8 \mathrm{e}+06$ & 4558.59 & \\
\hline Cr II 4588.199 & 6.77 & $1.20 \mathrm{e}+07$ & 4588.19 & B \\
\hline Cr II 4616.629 & 6.76 & $4.00 \mathrm{e}+06$ & 4616.64 & \\
\hline Cr II 4634.07 & 6.75 & $8.90 \mathrm{e}+06$ & 4634.08 & \\
\hline Cr II 4812.337 & 6.44 & $4.60 \mathrm{e}+05$ & 4812.32 & \\
\hline Cr II 4824.127 & 6.44 & $1.70 \mathrm{e}+06$ & 4824.1 & P, Wi-I \\
\hline Cr II 4848.235 & 6.42 & $2.60 \mathrm{e}+06$ & 4848.23 & P, Wi-I \\
\hline Cr II 4876.473 & 6.41 & $1.60 \mathrm{e}+06$ & 4876.49 & \\
\hline Cr II 5249.437 & 6.12 & $9.70 \mathrm{e}+04$ & 5249.33 & B \\
\hline Cr II 6053.47 & 6.79 & $1.60 \mathrm{e}+05$ & 6053.41 & B \\
\hline Mn I 3982.157 & 7.38 & $3.5 \mathrm{e}+07$ & 3982.11 & $\mathrm{~B}, \mathrm{~S} / \mathrm{N}$ \\
\hline Fe I 3589.452: & 6.18 & $1.05 \mathrm{e}+07$ & 3589.44 & $\mathrm{~S} / \mathrm{N}$ \\
\hline Fe I 3592.892: & 5.65 & $2.2 \mathrm{e}+05$ & 3592.81 & $\mathrm{~S} / \mathrm{N}$ \\
\hline Fe I 3595.864: & 5.9 & $4.14 \mathrm{e}+05$ & 3595.77 & $\mathrm{~S} / \mathrm{N}$ \\
\hline Fe I 3608.141/.174: & $6.29 / 6.43$ & $6.22 \mathrm{e}+06 /-$ & 3608.2 & $\mathrm{~S} / \mathrm{N}$ \\
\hline Fe I 3612.936: & 5.61 & $1.11 \mathrm{e}+07$ & 3612.99 & $\mathrm{~S} / \mathrm{N}$ \\
\hline Fe I 3616.32 & 5.85 & $6.86 e+05$ & 3616.43 & $\mathrm{~S} / \mathrm{N}$ \\
\hline
\end{tabular}


Table 3. continued.

\begin{tabular}{|c|c|c|c|c|}
\hline $\begin{array}{l}\text { Line } \lambda_{\text {lab }} \\
(\AA)\end{array}$ & $\begin{array}{c}E_{k} \\
(\mathrm{eV})\end{array}$ & $\begin{array}{c}A_{k i} \\
\left(\mathrm{~s}^{-1}\right) \\
\end{array}$ & $\begin{array}{l}\lambda_{\text {obs }} \\
(\AA) \\
\end{array}$ & Comments \\
\hline Fe I 3617.962 & 5.88 & - & 3618.04 & $\mathrm{~S} / \mathrm{N}$ \\
\hline Fe I 3647.408/.424 & $4.96 / 6.47$ & $-/ 3.38 \mathrm{e}+07$ & 3647.44 & $\mathrm{~B}, \mathrm{~S} / \mathrm{N}$ \\
\hline Fe I 3651.097 & 6.25 & $7.72 \mathrm{e}+05$ & 3651.06 & $\mathrm{~B}, \mathrm{~S} / \mathrm{N}$ \\
\hline Fe I 3674.406 & 6.61 & - & 3674.41 & $\mathrm{~S} / \mathrm{N}$ \\
\hline Fe I 3677.454/.503 & $7.25 / 6.67$ & $-1-$ & 3677.47 & $\mathrm{~S} / \mathrm{N}$ \\
\hline Fe I 3684.107/.137 & $6.09 / 6.67$ & $2.97 \mathrm{e}+07 / 9.29 \mathrm{e}+06$ & 3684.14 & $\mathrm{~S} / \mathrm{N}$ \\
\hline Fe I 3687.658 & 6.09 & $7.38 \mathrm{e}+06$ & 3687.66 & $\mathrm{~S} / \mathrm{N}$ \\
\hline Fe I 3727.527 & 6.74 & - & 3727.44 & $\mathrm{~S} / \mathrm{N}$ \\
\hline Fe I 3741.475 & 6.74 & - & 3741.48 & $\mathrm{~S} / \mathrm{N}$ \\
\hline Fe I 3742.944 & 6.74 & - & 3742.92 & $\mathrm{~S} / \mathrm{N}$ \\
\hline Fe I 3743.362 & 4.30 & $2.60 \mathrm{e}+07$ & 3743.37 & $\mathrm{~B}$ \\
\hline Fe I 3745.561/.471 Co I 3745.50 & $1.15 \mathrm{e}+07 /-$ & 3.4 & 3.209 & B \\
\hline Fe I 3748.209/.262 Ca I 3748.35 & $-/ 9.15 e+06$ & 6.7 & 3.167 & $\mathrm{~B}$ \\
\hline Fe I 3757.454 & 6.6 & $8.26 \mathrm{e}+06$ & 3757.54 & $\mathrm{~B}$ \\
\hline Fe I 3760.531 & 5.52 & $5.50 \mathrm{e}+06$ & 3760.51 & $\mathrm{~S} / \mathrm{N}$ \\
\hline Fe I 3764.208 & 5.49 & - & 3764.19 & $\mathrm{~S} / \mathrm{N}$ \\
\hline Fe I 3778.697 & 5.48 & $9.10 \mathrm{e}+05$ & 3778.69 & $\mathrm{~S} / \mathrm{N}$ \\
\hline Fe I 3779.202 & 6.04 & - & 3779.3 & $\mathrm{~S} / \mathrm{N}$ \\
\hline Fe I 3779.453 & 6.55 & $1.05 \mathrm{e}+07$ & 3779.5 & $\mathrm{~S} / \mathrm{N}$ \\
\hline Fe I 3787.88 & 4.28 & $1.29 \mathrm{e}+07$ & 3787.89 & $\mathrm{~S} / \mathrm{N}$ \\
\hline Fe I 3795.002 & 4.26 & $1.15 \mathrm{e}+07$ & 3795.04 & $\mathrm{~S} / \mathrm{N}$ \\
\hline Fe I 3797.515 & 6.5 & $4.57 \mathrm{e}+07$ & 3797.56 & $\mathrm{~B}, \mathrm{~S} / \mathrm{N}$ \\
\hline Fe I 3798.511 & 4.18 & $3.23 \mathrm{e}+06$ & 3798.51 & B \\
\hline Fe I 3799.547 & 4.22 & $7.31 \mathrm{e}+06$ & 3799.53 & $\mathrm{~B}$ \\
\hline Fe I 3813.933 & 5.68 & $2.91 \mathrm{e}+05$ & 3813.97 & $\mathrm{~S} / \mathrm{N}$ \\
\hline Fe I 3814.523 & 4.26 & $6.24 \mathrm{e}+05$ & 3814.48 & $\mathrm{~S} / \mathrm{N}$ \\
\hline Fe I 3815.84 & 4.73 & $1.12 \mathrm{e}+08$ & 3815.82 & $\mathrm{~S} / \mathrm{N}$ \\
\hline Fe I 3820.425 & 4.1 & $6.67 \mathrm{e}+07$ & 3820.44 & $\mathrm{~S} / \mathrm{N}$ \\
\hline Fe I 3824.556 & 5.93 & - & 3894.6 & $\mathrm{~S} / \mathrm{N}$ \\
\hline Fe I 3825.881 & 4.15 & $5.97 \mathrm{e}+07$ & 3825.9 & $\mathrm{~S} / \mathrm{N}$ \\
\hline Fe I 3827.571 & 5.93 & $1.56 \mathrm{e}+06$ & 3827.48 & $\mathrm{~S} / \mathrm{N}$ \\
\hline Fe I 3827.822 & 4.8 & $1.05 \mathrm{e}+08$ & 3827.81 & $\mathrm{~S} / \mathrm{N}$ \\
\hline Fe I 3834.222 & 4.19 & $4.52 \mathrm{e}+07$ & 3834.26 & $\mathrm{~B}, \mathrm{~S} / \mathrm{N}$ \\
\hline Fe I 3840.437 & 4.22 & $4.70 \mathrm{e}+07$ & 3840.45 & $\mathrm{~S} / \mathrm{N}$ \\
\hline Fe I 3842.896 & 5.81 & $4.87 \mathrm{e}+05$ & 3842.89 & SJ \\
\hline Fe I 3845.985 & 6.59 & $4.50 \mathrm{e}+06$ & 3846.08 & $\mathrm{~B}$ \\
\hline Fe I 3852.573 & 5.39 & $3.26 \mathrm{e}+06$ & 3852.58 & \\
\hline Fe I 3856.371 & 3.27 & $4.64 \mathrm{e}+06$ & 3856.29 & \\
\hline Fe I 3859.212 & 5.61 & $7.25 \mathrm{e}+06$ & 3859.18 & \\
\hline Fe I 3859.911 & 3.21 & $9.69 e+06$ & 3859.92 & \\
\hline Fe I 3865.523 & 4.22 & $1.55 \mathrm{e}+07$ & 3865.5 & \\
\hline Fe I 3872.501 & 4.19 & $1.05 \mathrm{e}+07$ & 3872.49 & \\
\hline Fe I 3878.018 & 4.15 & $7.72 \mathrm{e}+06$ & 3878.02 & \\
\hline Fe I 3878.573 & 3.28 & $6.17 \mathrm{e}+06$ & 3878.59 & \\
\hline Fe I 3895.656 & 3.29 & $9.39 \mathrm{e}+06$ & 3895.66 & \\
\hline Fe I 3897.890/8.009 & $5.87 / 4.19$ & $6.20 \mathrm{e}+06 / 8.00 \mathrm{e}+05$ & 3897.97 & \\
\hline Fe I 3920.257 & 3.28 & $2.60 \mathrm{e}+06$ & 3920.24 & \\
\hline Fe I 3925.644/.941 & $5.99 / 6.01$ & $8.04 \mathrm{e}+06 / 1.67 \mathrm{e}+07$ & 3925.79 & \\
\hline Fe I 3927.92 & 3.27 & $2.60 \mathrm{e}+06$ & 3927.91 & \\
\hline Fe I 3931.117 & 6.42 & $4.80 \mathrm{e}+06$ & 3931.2 & \\
\hline Fe I 3944.739 & 5.99 & $1.3 e+06$ & 3944.73 & \\
\hline
\end{tabular}


Table 3. continued.

\begin{tabular}{|c|c|c|c|c|}
\hline $\begin{array}{l}\text { Line } \lambda_{\text {lab }} \\
(\AA)\end{array}$ & $\begin{array}{c}E_{k} \\
(\mathrm{eV})\end{array}$ & $\begin{array}{c}A_{k i} \\
\left(\mathrm{~s}^{-1}\right)\end{array}$ & $\begin{array}{l}\lambda_{\mathrm{obs}} \\
(\AA)\end{array}$ & Comments \\
\hline Fe I 3949.953 & 5.31 & $4.79 \mathrm{e}+06$ & 3949.9 & $\mathrm{~S} / \mathrm{N}$ \\
\hline Fe I 3966.061 & 4.73 & $1.32 \mathrm{e}+06$ & 3966.0 & \\
\hline Fe I 3977.741 & 5.31 & $6.41 \mathrm{e}+06$ & 3977.71 & \\
\hline Fe I 3981.771 & 5.84 & $3.57 \mathrm{e}+06$ & 3981.75 & Ser \\
\hline Fe I 3997.392/.483/8.052 & $5.83 / 6.34 / 5.79$ & $1.26 \mathrm{e}+07 /-$ & 3997.49 & $\mathrm{Fe} \mathrm{I}, \mathrm{B}$ \\
\hline Fe I 4005.242 & 4.65 & $2.04 \mathrm{e}+07$ & 4005.22 & \\
\hline Fe I 4017.148/.083 & $6.13 / 5.84$ & $3.25 \mathrm{e}+06 / 8.42 \mathrm{e}+05$ & 4017.12 & $\mathrm{~B}$ \\
\hline Fe I 4018.267 & 6.35 & $3.44 \mathrm{e}+06$ & 4018.23 & $\mathrm{~B}$ \\
\hline $\mathrm{Fe}$ I 4021.866 & 5.84 & $8.55 e+06$ & 4021.86 & \\
\hline Fe I 4029.629 & 6.34 & - & 4029.58 & \\
\hline Fe I 4030.185 & 5.27 & $2.85 \mathrm{e}+05$ & 4030.07 & $\mathrm{~B}$ \\
\hline Fe I 4035.594 & 7.29 & - & 4035.54 & \\
\hline $\mathrm{Fe}$ I 4041.271 & 6.37 & - & 4041.09 & $\mathrm{~B}, \mathrm{~W}$ \\
\hline $\mathrm{Fe}$ I 4045.594 & 6.27 & $7.39 \mathrm{e}+06$ & 4045.55 & $\mathrm{P}$ \\
\hline $\mathrm{Fe}$ I 4051.905 & 6.46 & $3.30 \mathrm{e}+06$ & 4051.92 & W \\
\hline Fe I 4056.344 & 6.7 & - & 4056.25 & W \\
\hline $\mathrm{Fe}$ I 4063.594 & 4.61 & $6.65 e+07$ & 4063.57 & $\mathrm{~S}, \mathrm{P}, \mathrm{Wi}$ \\
\hline $\mathrm{Fe}$ I 4071.737 & 4.65 & $7.64 \mathrm{e}+07$ & 4071.77 & \\
\hline Fe I 4073.762 & 6.31 & $1.68 \mathrm{e}+07$ & 4073.74 & M \\
\hline $\mathrm{Fe}$ I 4074.786 & 6.09 & $3.43 e+06$ & 4074.82 & M \\
\hline $\mathrm{Fe}$ I 4084.491 & 6.37 & $8.66 e+06$ & 4084.49 & \\
\hline Fe I 4085.004 & 5.88 & $4.70 \mathrm{e}+06$ & 4085.01 & \\
\hline $\mathrm{Fe}$ I 4085.248 & 5.79 & - & 4085.25 & \\
\hline Fe I 4087.094 & 6.36 & $1.90 \mathrm{e}+06$ & 4087.05 & \\
\hline Fe I 4095.97 & 5.61 & $2.65 \mathrm{e}+06$ & 4095.98 & W \\
\hline $\mathrm{Fe}$ I 4107.488 & 5.85 & $1.74 \mathrm{e}+07$ & 4107.47 & \\
\hline Fe I 4109.056 & 6.31 & $3.62 \mathrm{e}+06$ & 4108.99 & \\
\hline $\mathrm{Fe}$ I 4109.801 & 5.86 & $1.51 \mathrm{e}+07$ & 4109.79 & \\
\hline $\mathrm{Fe}$ I 4112.958 & 7.19 & $1.10 \mathrm{e}+07$ & 4112.94 & W \\
\hline $\mathrm{Fe}$ I 4118.545 & 6.58 & $4.96 \mathrm{e}+07$ & 4118.57 & \\
\hline Fe I 4123.728/.766 & $6.00 / 5.61$ & $6.04 \mathrm{e}+05 /-$ & 4123.68 & M \\
\hline $\mathrm{Fe}$ I 4125.617 & 7.22 & $9.9 e+06$ & 4125.64 & \\
\hline Fe I 4126.183 & 6.34 & $4.20 \mathrm{e}+06$ & 4126.17 & \\
\hline Fe I 4132.057 & 4.61 & $1.18 \mathrm{e}+07$ & 4132.097 & $\mathrm{~S}, \mathrm{P}, \mathrm{Wi}$ \\
\hline Fe I 4136.998: & 6.41 & $2.75 \mathrm{e}+07$ & 4136.97 & \\
\hline Fe I 4139.927: & 3.98 & $1.83 \mathrm{e}+04$ & 4139.88 & \\
\hline Fe I 4143.414 & 6.04 & $2.70 \mathrm{e}+07$ & 4143.43 & S \\
\hline $\mathrm{Fe}$ I 4143.868 & 4.55 & $1.33 \mathrm{e}+07$ & 4143.84 & S \\
\hline Fe I 4147.668 & 4.47 & $4.36 \mathrm{e}+05$ & 4147.67 & \\
\hline Fe I 4152.169 & 3.94 & $3.24 \mathrm{e}+04$ & 4152.15 & $\mathrm{~S}$ \\
\hline Fe I 4153.899 & 6.38 & $2.05 \mathrm{e}+07$ & 4153.89 & MJ \\
\hline $\mathrm{Fe}$ I 4154.499 & 5.82 & $2.64 \mathrm{e}+07$ & 4154.49 & \\
\hline Fe I 4154.805 & 6.35 & $1.40 \mathrm{e}+07$ & 4154.8 & \\
\hline Fe I 4156.671/.768 & $5.93 / 5.81$ & $5.27 \mathrm{e}+05 /-$ & 4156.77 & \\
\hline Fe I 4158.792 & 6.41 & $1.60 \mathrm{e}+07$ & 4158.8 & \\
\hline $\mathrm{Fe}$ I 4163.676 & 5.67 & - & 4163.6 & \\
\hline Fe I 4168.614 & 6.34 & $6.80 \mathrm{e}+05$ & 4168.61 & \\
\hline Fe I 4173.315/.181 & $5.82 / 6.37$ & $2.63 \mathrm{e}+06 /-$ & 4173.26 & \\
\hline Fe I 4181.754 & 5.8 & $2.32 \mathrm{e}+07$ & 4181.74 & \\
\hline Fe I 4182.382 & 5.98 & $5.04 \mathrm{e}+06$ & 4182.38 & \\
\hline Fe I 4184.892 & 5.79 & $1.03 \mathrm{e}+07$ & 4184.88 & \\
\hline
\end{tabular}


Table 3. continued.

\begin{tabular}{|c|c|c|c|c|}
\hline $\begin{array}{l}\text { Line } \lambda_{\text {lab }} \\
(\AA)\end{array}$ & $\begin{array}{c}E_{k} \\
(\mathrm{eV})\end{array}$ & $\begin{array}{c}A_{k i} \\
\left(\mathrm{~s}^{-1}\right)\end{array}$ & $\begin{array}{l}\lambda_{\text {obs }} \\
(\AA) \\
\end{array}$ & Comments \\
\hline Fe I 4187.039 & 5.41 & $2.15 \mathrm{e}+07$ & 4187.04 & \\
\hline Fe I 4187.587 & 6.39 & $4.48 \mathrm{e}+06$ & 4187.41 & B \\
\hline Fe I 4187.795 & 5.39 & $1.52 \mathrm{e}+07$ & 4187.72 & \\
\hline Fe I 4191.43 & 5.43 & $2.73 e+07$ & 4191.4 & $\mathrm{P}$ \\
\hline Fe I 4195.329 & 6.29 & $1.11 \mathrm{e}+07$ & 4195.33 & B \\
\hline Fe I 4195.618 & 5.97 & $1.21 \mathrm{e}+06$ & 4195.58 & B \\
\hline Fe I 4196.208 & 6.35 & $1.09 \mathrm{e}+07$ & 4196.21 & $\mathrm{~B}$ \\
\hline Fe I 4196.671 & 5.39 & - & 4196.69 & B \\
\hline Fe I 4198.247/.304 & $6.32 / 5.35$ & $1.47 \mathrm{e}+07 / 8.03 \mathrm{e}+06$ & 4198.27 & $\mathrm{~B}$ \\
\hline Fe I 4198.634 & 6.37 & $1.25 \mathrm{e}+07$ & 4198.66 & $\mathrm{~B}$ \\
\hline Fe I 4199.095 & 6.0 & $4.92 \mathrm{e}+07$ & 4199.07 & $\mathrm{~B}$ \\
\hline Fe I 4199.983 & 3.04 & - & 4199.9 & WA, SJ \\
\hline Fe I 4202.029 & 4.43 & $8.22 \mathrm{e}+06$ & 4202.02 & $\mathrm{P}, \mathrm{Wi}$ \\
\hline Fe I 4203.938 & 6.58 & $2.97 \mathrm{e}+06$ & 4203.95 & $\mathrm{M}$ \\
\hline Fe I 4205.538 & 6.36 & $2.77 \mathrm{e}+06$ & 4205.46 & \\
\hline Fe I 4206.696 & 3.0 & $5.90 \mathrm{e}+03$ & 4206.6 & \\
\hline Fe I 4207.127 & 5.78 & $4.80 \mathrm{e}+06$ & 4207.11 & \\
\hline Fe I 4208.604 & 6.34 & - & 4208.6 & W \\
\hline Fe I 4210.343 & 5.43 & $1.48 \mathrm{e}+07$ & 4210.32 & \\
\hline $\mathrm{Fe}$ I $4215.423 / .477$ & $5.93 / 5.93$ & $5.98 \mathrm{e}+05 /-$ & 4215.51 & $\mathrm{P}$ \\
\hline Fe I 4216.184 & 2.94 & $1.84 \mathrm{e}+04$ & 4216.16 & \\
\hline Fe I 4219.36 & 6.51 & $2.88 \mathrm{e}+07$ & 4219.37 & B \\
\hline Fe I 4219.414 & 5.93 & $5.29 \mathrm{e}+05$ & 4219.41 & $\mathrm{~B}$ \\
\hline Fe I 4220.341 & 6.01 & $1.83 \mathrm{e}+07$ & 4220.36 & B \\
\hline $\mathrm{Fe} \mathrm{I} 4222.213$ & 5.39 & $5.76 \mathrm{e}+06$ & 4222.2 & \\
\hline Fe I 4224.171 & 6.3 & $1.06 \mathrm{e}+07$ & 4224.19 & M \\
\hline Fe I 4226.343 & 5.39 & - & 4226.29 & \\
\hline Fe I 4227.426 & 6.26 & $5.29 \mathrm{e}+07$ & 4227.36 & M \\
\hline Fe I 4235.937 & 5.35 & $1.88 \mathrm{e}+07$ & 4235.91 & M \\
\hline Fe I 4238.81 & 6.32 & $2.41 \mathrm{e}+07$ & 4238.78 & \\
\hline Fe I 4247.306/.425 & $5.37 / 6.29$ & $1.02 \mathrm{e}+05 / 1.94 \mathrm{e}+07$ & 4247.39 & $\mathrm{M}$ \\
\hline Fe I 4250.119 & 5.39 & $2.07 \mathrm{e}+07$ & 4250.1 & Wer \\
\hline Fe I 4250.787 & 4.47 & $1.02 \mathrm{e}+07$ & 4250.77 & \\
\hline Fe I 4253.93 & 6.55 & - & 4253.93 & $\mathrm{~W}, \mathrm{NJ}$ \\
\hline $\mathrm{Fe} \mathrm{I} 4258.611$ & 5.74 & $8.0 \mathrm{e}+05$ & 4258.64 & B \\
\hline Fe I 4259.999 & 6.24 & $1.57 \mathrm{e}+06$ & 4259.98 & $\mathrm{~B}$ \\
\hline Fe I 4260.08 & 6.31 & - & 4260.06 & WJ \\
\hline Fe I 4260.474 & 5.31 & $3.99 \mathrm{e}+07$ & 4260.45 & \\
\hline Fe I 4266.964 & 5.63 & $6.27 \mathrm{e}+05$ & 4266.97 & \\
\hline Fe I 4267.826 & 6.02 & $8.17 \mathrm{e}+06$ & 4267.81 & \\
\hline Fe I 4271.153 & 5.35 & $1.82 \mathrm{e}+07$ & 4271.17 & MJ \\
\hline Fe I 4271.76 & 4.39 & $2.28 \mathrm{e}+07$ & 4271.73 & \\
\hline Fe I 4278.231 & 6.27 & $1.00 \mathrm{e}+06$ & 4278.09 & \\
\hline Fe I 4282.403 & 5.07 & $1.21 \mathrm{e}+07$ & 4282.41 & \\
\hline Fe I 4285.442 & 6.13 & $1.78 \mathrm{e}+06$ & 4285.44 & \\
\hline Fe I 4289.914 & 6.29 & - & 4289.85 & B \\
\hline Fe I 4290.864 & 5.72 & $3.83 \mathrm{e}+05$ & 4290.8 & M \\
\hline Fe I 4293.798 & 5.32 & - & 4293.82 & M \\
\hline Fe I 4324.948/Cr I 4325.08 & $5.06 / 5.83$ & $-1-$ & 4325.0 & \\
\hline Fe I 4325.739/.762 & $2.87 / 4.47$ & $-/ 5.16 e+07$ & 4325.74 & \\
\hline Fe I 4326.753 & 5.81 & $4.63 \mathrm{e}+05$ & 4326.6 & M \\
\hline
\end{tabular}


Table 3. continued.

\begin{tabular}{|c|c|c|c|c|}
\hline $\begin{array}{l}\text { Line } \lambda_{\text {lab }} \\
(\AA)\end{array}$ & $\begin{array}{c}E_{k} \\
(\mathrm{eV})\end{array}$ & $\begin{array}{c}A_{k i} \\
\left(\mathrm{~s}^{-1}\right)\end{array}$ & $\begin{array}{l}\lambda_{\mathrm{obs}} \\
(\AA)\end{array}$ & Comments \\
\hline Fe I 4358.499 & 5.79 & $8.09 \mathrm{e}+05$ & 4358.5 & \\
\hline Fe I 4367.903 & 4.45 & $9.09 \mathrm{e}+04$ & 4367.9 & \\
\hline Fe I 4369.771 & 5.88 & $6.09 \mathrm{e}+06$ & 4369.77 & $\mathrm{M}$ \\
\hline Fe I 4375.930/.986 & $2.83 / 5.88$ & $2.95 \mathrm{e}+04 /-$ & 4375.9 & $\mathrm{P}$ \\
\hline Fe I 4383.545 & 4.31 & $5.00 \mathrm{e}+07$ & 4383.53 & \\
\hline Fe I 4390.95 & 5.84 & $1.16 \mathrm{e}+06$ & 4390.95 & \\
\hline Fe I 4404.75 & 4.37 & $2.75 \mathrm{e}+07$ & 4404.72 & \\
\hline Fe I 4405.019 & 2.87 & $2.95 \mathrm{e}+02$ & 4405.02 & \\
\hline Fe I 4408.413 & 5.01 & $1.92 \mathrm{e}+06$ & 4408.37 & \\
\hline Fe I 4409.119 & 6.11 & $7.40 \mathrm{e}+05$ & 4409.09 & \\
\hline Fe I 4415.122 & 4.42 & $1.19 \mathrm{e}+07$ & 4415.12 & SA, M \\
\hline Fe I 4422.568 & 5.65 & $8.72 \mathrm{e}+06$ & 4422.56 & \\
\hline Fe I 4427.298/.31 & $6.45 / 2.45$ & $-/ 3.41 \mathrm{e}+04$ & 4427.28 & \\
\hline Fe I 4430.189 & 5.82 & $2.00 \mathrm{e}+06$ & 4430.22 & \\
\hline Fe I 4430.614 & 5.02 & $7.45 e+06$ & 4430.61 & \\
\hline Fe I 4435.148 & 2.88 & $4.72 \mathrm{e}+03$ & 4435.16 & B \\
\hline Fe I 4442.339 & 4.99 & $3.76 \mathrm{e}+06$ & 4442.35 & B \\
\hline Fe I 4443.194 & 5.86 & $1.02 \mathrm{e}+07$ & 4443.11 & B \\
\hline Fe I 4447.717 & 5.01 & $5.11 \mathrm{e}+06$ & 4447.69 & \\
\hline Fe I 4454.381 & 5.61 & $3.38 \mathrm{e}+06$ & 4454.41 & \\
\hline Fe I 4459.117 & 4.96 & $2.52 \mathrm{e}+06$ & 4459.1 & \\
\hline Fe I 4461.652 & 2.87 & $2.95 \mathrm{e}+04$ & 4461.6 & $S$ \\
\hline Fe I 4466.551 & 5.61 & $1.20 \mathrm{e}+07$ & 4466.54 & \\
\hline Fe I 4476.018 & 5.61 & $1.01 \mathrm{e}+07$ & 4476.03 & \\
\hline Fe I 4482.169 & 2.88 & $2.09 \mathrm{e}+04$ & 4482.18 & \\
\hline Fe I 4489.739 & 2.88 & $1.19 \mathrm{e}+04$ & 4489.72 & \\
\hline Fe I 4494.464 & 5.71 & - & 4494.48 & \\
\hline Fe I 4528.614 & 4.91 & $5.44 \mathrm{e}+06$ & 4528.62 & \\
\hline Fe I 4533.956/Ti II 4533.97 & $5.68 / 3.97$ & $-/ 9.20 \mathrm{e}+06$ & 4533.96 & \\
\hline Fe I 4583.721 & 5.82 & - & 4583.66 & P, Wi-I \\
\hline Fe I 4592.651 & 4.26 & $1.61 \mathrm{e}+05$ & 4592.61 & \\
\hline Fe I 4602.00 & 4.3 & $7.36 \mathrm{e}+04$ & 4602.0 & \\
\hline Fe I 4602.941 & 4.18 & $1.72 \mathrm{e}+05$ & 4602.92 & \\
\hline Fe I 4607.647 & 5.96 & - & 4607.65 & $\mathrm{~W}$ \\
\hline Fe I 4611.185 & 5.54 & $6.64 \mathrm{e}+04$ & 4611.21 & \\
\hline Fe I $4618.757 / \mathrm{Cr}$ II 4618.803 & $5.63 / 6.76$ & $1.36 e+05 / 6.1 e+06$ & 4618.78 & \\
\hline Fe I 4619.288 & 6.29 & $5.20 \mathrm{e}+06$ & 4619.28 & \\
\hline Fe I 4625.045 & 5.92 & - & 4625.04 & \\
\hline Fe I 4638.01 & 6.27 & $3.37 \mathrm{e}+06$ & 4637.99 & \\
\hline Fe I 4643.463 & 6.32 & $4.41 \mathrm{e}+06$ & 4643.43 & \\
\hline Fe I 4647.434 & 5.62 & $1.25 \mathrm{e}+06$ & 4647.43 & \\
\hline Fe I 4654.605/.628 & $6.26 / 5.87$ & $-1-$ & 4654.54 & SA, M \\
\hline Fe I 4667.453 & 6.25 & $6.03 e+06$ & 4667.62 & M \\
\hline Fe I 4673.163 & 6.31 & $3.81 \mathrm{e}+06$ & 4673.16 & W \\
\hline Fe I 4678.846 & 6.25 & $4.97 \mathrm{e}+06$ & 4678.85 & \\
\hline Fe I 4680.294 & 4.26 & $7.32 \mathrm{e}+03$ & 4680.28 & \\
\hline Fe I 4683.56 & 5.48 & $2.92 \mathrm{e}+05$ & 4683.55 & \\
\hline Fe I 4688.176 & 7.25 & - & 4688.22 & $\mathrm{~S} / \mathrm{N}, \mathrm{W}$ \\
\hline Fe I 4690.138 & 6.33 & $2.29 \mathrm{e}+06$ & 4690.22 & \\
\hline $\mathrm{Fe}$ I 4691.412 & 5.63 & $1.01 \mathrm{e}+06$ & 4691.33 & \\
\hline Fe I 4702.913 & 5.87 & - & 4702.98 & $\mathrm{P}$ \\
\hline
\end{tabular}


Table 3. continued.

\begin{tabular}{|c|c|c|c|c|}
\hline $\begin{array}{l}\text { Line } \lambda_{\text {lab }} \\
(\AA)\end{array}$ & $\begin{array}{c}E_{k} \\
(\mathrm{eV})\end{array}$ & $\begin{array}{c}A_{k i} \\
\left(\mathrm{~s}^{-1}\right) \\
\end{array}$ & $\begin{array}{l}\lambda_{\text {obs }} \\
(\AA) \\
\end{array}$ & Comments \\
\hline Fe I 4707.274 & 5.87 & - & 4707.31 & $\mathrm{M}$ \\
\hline Fe I 4708.968 & 6.27 & $3.98 \mathrm{e}+05$ & 4708.93 & M \\
\hline Fe I 4710.283 & 5.65 & $1.05 \mathrm{e}+06$ & 4710.24 & M \\
\hline Fe I 4714.359 & 7.24 & - & 4714.37 & \\
\hline Fe I 4727.395 & 6.31 & $6.85 \mathrm{e}+06$ & 4727.41 & M \\
\hline Fe I 4728.545 & 6.27 & $2.87 \mathrm{e}+06$ & 4728.53 & M \\
\hline Fe I 4736.773 & 5.83 & $4.78 \mathrm{e}+06$ & 4736.8 & \\
\hline Fe I 4741.529 & 5.45 & $7.29 \mathrm{e}+05$ & 4741.53 & \\
\hline Fe I 4745.80 & 6.27 & $2.27 \mathrm{e}+06$ & 4745.78 & \\
\hline Fe I 4757.578 & 5.88 & $5.10 \mathrm{e}+06$ & 4757.54 & \\
\hline Fe I 4768.32 & 6.29 & $1.13 \mathrm{e}+05$ & 4768.34 & \\
\hline Fe I 4771.696 & 4.8 & $3.42 \mathrm{e}+04$ & 4771.59 & $\mathrm{~B}$ \\
\hline Fe I $4772.803 / .83$ & $4.15 / 5.61$ & $5.30 \mathrm{e}+04 /-$ & 4772.8 & \\
\hline Fe I 4786.807 & 5.61 & $1.03 \mathrm{e}+06$ & 4786.76 & \\
\hline Fe I $4843.144 / \mathrm{Ni}$ I 4843.158 & $5.96 / 4.23$ & $9.20 \mathrm{e}+05 / 2.90 \mathrm{e}+04$ & 4843.15 & $\mathrm{pFe} I$ \\
\hline Fe I 4844.014 & 6.11 & $3.62 \mathrm{e}+05$ & 4844.01 & \\
\hline Fe I 4871.318 & 5.41 & $2.44 \mathrm{e}+07$ & 4871.31 & \\
\hline Fe I 4872.138 & 5.43 & $2.54 \mathrm{e}+07$ & 4872.1 & \\
\hline Fe I 4890.755 & 5.41 & $2.25 \mathrm{e}+07$ & 4890.75 & \\
\hline Fe I 4891.492 & 5.39 & $3.08 \mathrm{e}+07$ & 4891.47 & \\
\hline Fe I 4918.994 & 5.39 & $1.79 \mathrm{e}+07$ & 4919.01 & \\
\hline Fe I 4920.503 & 5.35 & $3.58 \mathrm{e}+07$ & 4920.5 & \\
\hline Fe I 4938.813 & 5.39 & $3.27 \mathrm{e}+06$ & 4938.81 & \\
\hline Fe I 4939.239 & 6.66 & - & 4939.25 & B \\
\hline Fe I 4939.687 & 3.37 & $1.39 \mathrm{e}+04$ & 4939.65 & \\
\hline Fe I 4946.388 & 5.87 & - & 4946.39 & W \\
\hline Fe I 4950.105 & 5.92 & - & 4950.1 & W \\
\hline Fe I 4957.298 & 5.35 & $1.18 \mathrm{e}+07$ & 4957.29 & $\mathrm{P}$ \\
\hline Fe I 4957.597 & 5.31 & $4.22 \mathrm{e}+07$ & 4957.59 & $\mathrm{P}$ \\
\hline Fe I 4962.571 & 6.68 & $1.37 \mathrm{e}+06$ & 4962.62 & \\
\hline Fe I 4966.088 & 5.83 & $3.31 \mathrm{e}+06$ & 4966.08 & \\
\hline Fe I 4982.50 & 6.59 & - & 4982.5 & \\
\hline Fe I 4983.25 & 6.64 & - & 4983.26 & \\
\hline Fe I 4983.853 & 6.59 & - & 4983.88 & \\
\hline Fe I 4985.252 & 6.41 & $1.48 \mathrm{e}+07$ & 4985.2 & \\
\hline Fe I 4985.547 & 5.35 & $1.39 \mathrm{e}+06$ & 4985.5 & \\
\hline Fe I 4988.95 & 6.64 & $5.20 \mathrm{e}+06$ & 4988.94 & W \\
\hline Fe I 4994.129 & 3.4 & $3.18 \mathrm{e}+04$ & 4994.08 & \\
\hline Fe I 5006.119 & 5.31 & $5.87 \mathrm{e}+06$ & 5006.12 & $\mathrm{~B}$ \\
\hline Fe I 5012.068 & 3.33 & $5.50 \mathrm{e}+04$ & 5012.07 & \\
\hline Fe I 5027.123 & 6.62 & - & 5027.1 & \\
\hline Fe I 5030.778 & 5.7 & $2.60 \mathrm{e}+04$ & 5030.75 & \\
\hline Fe I 5041.071 & 3.42 & $4.29 \mathrm{e}+04$ & 5041.07 & \\
\hline Fe I 5041.756 & 3.94 & $2.35 \mathrm{e}+05$ & 5041.74 & \\
\hline Fe I 5049.819 & 4.73 & $1.65 \mathrm{e}+06$ & 5049.82 & \\
\hline Fe I 5051.634 & 3.37 & $4.65 \mathrm{e}+04$ & 5051.52 & \\
\hline Fe I 5055.991 & 6.76 & $3.0 \mathrm{e}+05$ & 5055.99 & \\
\hline Fe I 5060.034 & 6.75 & - & 5060.03 & \\
\hline Fe I 5064.58/Ti I 5064.66 & $6.52 / 2.50$ & $-/ 3.79 e+06$ & 5064.66 & $\mathrm{~B}$ \\
\hline Fe I 5064.953/.018 & $6.70 / 6.7$ & $-/-$ & 5065.04 & \\
\hline Fe I 5068.766 & 5.39 & $3.37 \mathrm{e}+06$ & 5068.73 & $\mathrm{~B}$ \\
\hline
\end{tabular}


Table 3. continued.

\begin{tabular}{|c|c|c|c|c|}
\hline $\begin{array}{l}\text { Line } \lambda_{\text {lab }} \\
(\AA)\end{array}$ & $\begin{array}{c}E_{k} \\
(\mathrm{eV})\end{array}$ & $\begin{array}{c}A_{k i} \\
\left(\mathrm{~s}^{-1}\right)\end{array}$ & $\begin{array}{l}\lambda_{\mathrm{obs}} \\
(\AA)\end{array}$ & Comments \\
\hline Fe I 5072.078 & 6.73 & - & 5072.15 & \\
\hline Fe I 5074.748 & 6.66 & $1.40 \mathrm{e}+07$ & 5074.71 & \\
\hline Fe I 5076.264 & 6.74 & - & 5076.31 & \\
\hline Fe I 5079.223 & 4.64 & $7.38 \mathrm{e}+05$ & 5079.21 & \\
\hline Fe I 5079.74 & 3.43 & $5.19 \mathrm{e}+04$ & 5079.75 & \\
\hline Fe I 5083.338 & 3.40 & $4.06 \mathrm{e}+04$ & 5083.32 & \\
\hline Fe I 5090.773 & 6.69 & $1.90 \mathrm{e}+07$ & 5090.76 & \\
\hline Fe I 5098.572:/.698 & $6.36 / 4.61$ & $-/ 4.83 e+05$ & 5098.64 & \\
\hline Fe I 5104.191 & 6.61 & $2.7 \mathrm{e}+05$ & 5104.19 & B \\
\hline Fe I 5107.641 & 3.98 & $1.95 \mathrm{e}+05$ & 5107.51 & \\
\hline Fe I 5109.652 & 6.73 & - & 6509.66 & \\
\hline Fe I 5110.358 & 6.0 & $9.99 \mathrm{e}+05$ & 5110.34 & \\
\hline Fe I 5123.72 & 3.43 & $7.24 \mathrm{e}+04$ & 5123.66 & \\
\hline Fe I 5124.62 & 5.72 & - & 5124.66 & \\
\hline Fe I 5126.193 & 6.67 & $3.20 \mathrm{e}+06$ & 5126.18 & W \\
\hline Fe I 5139.251 & 5.41 & $9.16 \mathrm{e}+06$ & 5139.31 & \\
\hline Fe I 5141.739 & 4.83 & $4.86 \mathrm{e}+05$ & 5141.74 & \\
\hline Fe I 5150.839 & 3.40 & $3.30 \mathrm{e}+04$ & 5150.82 & \\
\hline Fe I 5151.911 & 3.42 & $2.39 \mathrm{e}+04$ & 5151.91 & \\
\hline Fe I 5162.272 & 6.58 & - & 5162.24 & \\
\hline Fe I 5167.488 & 3.88 & $2.72 \mathrm{e}+06$ & 5167.55 & \\
\hline Fe I 5191.455 & 5.43 & $2.32 \mathrm{e}+07$ & 5191.47 & \\
\hline Fe I 5192.344 & 5.39 & $1.34 \mathrm{e}+07$ & 5192.34 & \\
\hline Fe I 5194.941 & 3.94 & $2.87 \mathrm{e}+05$ & 5194.92 & M \\
\hline Fe I 5202.256 & 6.64 & - & 5202.24 & \\
\hline Fe I 5216.274 & 3.98 & $3.47 e+05$ & 5216.26 & \\
\hline Fe I 5227.150/.189 & $4.80 / 3.93$ & $-/ 2.89 \mathrm{e}+06$ & 5227.16 & \\
\hline Fe I 5242.491 & 6.0 & $2.38 \mathrm{e}+06$ & 5242.38 & W \\
\hline Fe I 5247.05 & 2.45 & $3.92 \mathrm{e}+02$ & 5247.03 & \\
\hline Fe I 5250.646 & 4.56 & $2.28 \mathrm{e}+05$ & 5250.54 & B \\
\hline Fe I 5251.965 & 5.93 & - & 5251.95 & \\
\hline Fe I 5254.955 & 2.47 & $8.31 \mathrm{e}+02$ & 5254.9 & \\
\hline Fe I 5263.306 & 5.62 & $6.36 \mathrm{e}+06$ & 5263.32 & \\
\hline Fe I 5266.555 & 5.35 & $1.10 \mathrm{e}+07$ & 5266.54 & \\
\hline Fe I 5269.537 & 3.21 & $1.27 \mathrm{e}+06$ & 5269.52 & \\
\hline Fe I 5270.356 & 3.96 & $3.67 \mathrm{e}+06$ & 5270.31 & \\
\hline Fe I 5302.299 & 5.62 & $9.04 \mathrm{e}+06$ & 5302.27 & \\
\hline Fe I 5307.361 & 3.94 & $3.48 \mathrm{e}+04$ & 5307.34 & \\
\hline Fe I 5324.179 & 5.54 & $2.06 \mathrm{e}+07$ & 5324.16 & \\
\hline Fe I 5328.038 & 3.24 & $1.15 \mathrm{e}+06$ & 5328.05 & \\
\hline Fe I 5328.531 & 3.88 & $4.74 \mathrm{e}+05$ & 5328.5 & \\
\hline Fe I 5332.899 & 3.88 & $4.36 \mathrm{e}+04$ & 5332.85 & \\
\hline Fe I 5339.929 & 5.59 & $6.36 \mathrm{e}+06$ & 5339.91 & \\
\hline Fe I 5341.024 & 3.93 & $5.21 \mathrm{e}+05$ & 5341.01 & \\
\hline Fe I 5353.373/Ni I 5353.391 & $6.42 / 4.27$ & $-/ 1.20 \mathrm{e}+05$ & 5353.37 & \\
\hline Fe I 5371.489 & 3.27 & $1.05 \mathrm{e}+06$ & 5371.45 & $\mathrm{P}$ \\
\hline Fe I 5383.369 & 6.61 & $7.81 \mathrm{e}+07$ & 5383.35 & \\
\hline Fe I 5389.479 & 6.72 & - & 5389.47 & \\
\hline Fe I 5393.167 & 5.54 & $4.91 \mathrm{e}+06$ & 5393.19 & \\
\hline Fe I 5397.127 & 3.21 & $2.58 \mathrm{e}+05$ & 5397.11 & \\
\hline Fe I 5446.874 & 3.88 & $2.50 \mathrm{e}+04$ & 5446.0 & $\mathrm{P}$ \\
\hline
\end{tabular}


Table 3. continued.

\begin{tabular}{|c|c|c|c|c|}
\hline $\begin{array}{l}\text { Line } \lambda_{\text {lab }} \\
(\AA)\end{array}$ & $\begin{array}{c}E_{k} \\
(\mathrm{eV})\end{array}$ & $\begin{array}{c}A_{k i} \\
\left(\mathrm{~s}^{-1}\right)\end{array}$ & $\begin{array}{l}\lambda_{\mathrm{obs}} \\
(\AA)\end{array}$ & Comments \\
\hline Fe I 5455.609 & 3.28 & $6.05 \mathrm{e}+05$ & 5455.56 & $\mathrm{P}$ \\
\hline Fe I 5487.738/.746 & $4.43 / 6.58$ & $2.49 \mathrm{e}+05 /-$ & 5487.74 & \\
\hline Fe I 5497.516 & 3.27 & $6.25 \mathrm{e}+04$ & 5497.47 & \\
\hline Fe I 5501.465 & 3.21 & $2.20 \mathrm{e}+04$ & 5501.45 & \\
\hline Fe I 5506.779 & 3.24 & $5.01 \mathrm{e}+04$ & 5506.76 & \\
\hline Fe I 5508.408 & 7.21 & - & 5508.49 & \\
\hline Fe I 5565.704/.685 & $6.83 / 2.28$ & $-1-$ & 5565.66 & $\mathrm{~B}$ \\
\hline Fe I 5567.278/.391 & $6.64 / 4.83$ & $-/ 1.53 \mathrm{e}+05$ & 5567.32 & \\
\hline Fe I 5569.618 & 5.64 & $2.34 \mathrm{e}+07$ & 5569.6 & \\
\hline Fe I 5572.842 & 5.62 & $2.28 \mathrm{e}+07$ & 5572.83 & SA \\
\hline Fe I 5576.088 & 5.65 & $2.50 \mathrm{e}+07$ & 5576.08 & \\
\hline Fe I 5586.755 & 5.59 & $2.19 \mathrm{e}+07$ & 5586.75 & \\
\hline Fe I 5592.234 & 5.85 & - & 5592.24 & \\
\hline Fe I 5594.655 & 6.76 & $5.20 \mathrm{e}+06$ & 5594.71 & \\
\hline Fe I 5615.644 & 5.54 & $2.64 \mathrm{e}+07$ & 5615.59 & $\mathrm{P}$ \\
\hline Fe I 5624.542 & 5.62 & $7.41 \mathrm{e}+06$ & 5624.52 & \\
\hline Fe I 5633.946 & 7.19 & $7.70 \mathrm{e}+06$ & 5633.94 & \\
\hline Fe I 5638.262 & 6.42 & $4.40 \mathrm{e}+06$ & 5638.24 & \\
\hline Fe I 5640.876 & 6.41 & - & 5640.94 & \\
\hline Fe I 5658.816 & 5.59 & $4.34 \mathrm{e}+06$ & 5658.78 & \\
\hline Fe I 5662.516 & 6.37 & $6.18 \mathrm{e}+06$ & 5662.5 & $\mathrm{~B}$ \\
\hline Fe I 5667.518/.460/.666 & $6.36 / 7.25 / 4.8$ & $6.12 \mathrm{e}+05 /-$ & 5667.49 & $\mathrm{~B}$ \\
\hline Fe I 5679.022 & 6.83 & $3.7 \mathrm{e}+06$ & 5678.98 & W \\
\hline Fe I 5684.484/Si I 5684.484 & $7.25 / 7.13$ & $2.60 \mathrm{e}+06 / 2.60 \mathrm{e}+06$ & 5684.62 & $\mathrm{~S} / \mathrm{N}, \mathrm{T}$ \\
\hline Fe I 5693.318 & 7.17 & - & 5693.33 & \\
\hline Fe I 5701.544 & 4.73 & $1.78 \mathrm{e}+05$ & 5701.53 & \\
\hline Fe I 5705.992 & 6.78 & $6.10 \mathrm{e}+06$ & 5705.99 & \\
\hline Fe I 5709.378 & 5.54 & $2.13 \mathrm{e}+06$ & 5709.44 & \\
\hline Fe I 5717.833 & 6.45 & $5.40 \mathrm{e}+06$ & 5717.86 & W \\
\hline Fe I 5731.762 & 6.42 & $1.60 \mathrm{e}+06$ & 5731.81 & W \\
\hline Fe I 5732.295 & 7.15 & - & 5732.31 & \\
\hline Fe I 5753.122 & 6.41 & $8.26 \mathrm{e}+06$ & 5753.12 & \\
\hline Fe I 5753.693 & 6.46 & - & 5753.65 & \\
\hline Fe I 5755.348 & 5.79 & - & 5755.36 & \\
\hline Fe I 5775.080/.054 & $6.37 / 2.2$ & $1.12 \mathrm{e}+06 /-$ & 5775.04 & \\
\hline Fe I 5780.60 & 5.39 & $7.60 \mathrm{e}+04$ & 5780.62 & \\
\hline Fe I 5782.109 & 7.21 & - & 5782.09 & \\
\hline Fe I 5783.895 & 5.43 & - & 5783.88 & $\mathrm{~S} / \mathrm{N}$ \\
\hline Fe I 5791.018 & 5.35 & $1.29 \mathrm{e}+05$ & 5790.97 & \\
\hline Fe I 5804.035 & 6.02 & $1.60 \mathrm{e}+05$ & 5804.06 & \\
\hline Fe I 5805.757 & 7.17 & - & 5805.84 & \\
\hline Fe I 5809.155 & 5.83 & - & 5808.98 & \\
\hline Fe I 5816.373/.061 & $6.68 / 6.43$ & $4.49 \mathrm{e}+06 /-$ & 5816.33 & B \\
\hline Fe I 5835.573/.496 & $4.96 / 6.38$ & $-/-$ & 5835.52 & B \\
\hline Fe I 5853.682 & 6.31 & - & 5853.56 & \\
\hline Fe I 5862.356 & 6.66 & - & 5862.2 & \\
\hline Fe I 5883.816 & 6.07 & $1.90 \mathrm{e}+06$ & 5883.83 & \\
\hline Fe I 5914.112/.201 & 6.7 & $-/-$ & 5914.17 & \\
\hline Fe I 5916.247 & 4.55 & $2.15 \mathrm{e}+04$ & 5916.21 & $\mathrm{~S} / \mathrm{N}, \mathrm{T}$ \\
\hline Fe I 5930.18 & 6.74 & - & 5930.15 & $\mathrm{~B}, \mathrm{P}$ \\
\hline Fe I 5931.89 & 6.17 & - & 5931.82 & $\mathrm{M}, \mathrm{S} / \mathrm{N}$ \\
\hline
\end{tabular}


Table 3. continued.

\begin{tabular}{|c|c|c|c|c|}
\hline $\begin{array}{l}\text { Line } \lambda_{\text {lab }} \\
(\AA)\end{array}$ & $\begin{array}{c}E_{k} \\
(\mathrm{eV})\end{array}$ & $\begin{array}{c}A_{k i} \\
\left(\mathrm{~s}^{-1}\right)\end{array}$ & $\begin{array}{l}\lambda_{\mathrm{obs}} \\
(\AA)\end{array}$ & Comments \\
\hline Fe I 5934.655 & 6.02 & $2.00 \mathrm{e}+06$ & 5934.62 & \\
\hline Fe I 5952.718 & 6.07 & $1.50 \mathrm{e}+06$ & 5952.56 & \\
\hline Fe I 5956.694 & 2.94 & $5.15 \mathrm{e}+02$ & 5956.64 & \\
\hline Fe I 5958.333 & 4.26 & - & 5958.41 & SA, MJ \\
\hline Fe I 5976.777 & 6.02 & - & 5976.71 & \\
\hline Fe I 5983.681 & 6.62 & - & 5983.74 & \\
\hline Fe I 5984.815 & 6.8 & - & 5984.83 & \\
\hline Fe I 6003.012 & 5.95 & - & 6003.03 & \\
\hline Fe I 6007.959 & 6.72 & - & 6007.95 & \\
\hline Fe I 6008.556 & 5.95 & - & 6008.54 & \\
\hline Fe I 6020.169 & 6.67 & - & 6020.12 & \\
\hline Fe I 6021.791 & 4.26 & - & 6021.79 & \\
\hline Fe I 6024.058 & 6.61 & - & 6024.03 & \\
\hline Fe I 6027.05 & 6.13 & $1.36 \mathrm{e}+06$ & 6027.07 & \\
\hline Fe I 6065.482 & 4.65 & $1.07 \mathrm{e}+06$ & 6065.52 & P, Wi-I \\
\hline Fe I 6078.491 & 6.83 & - & 6078.46 & \\
\hline Fe I 6091.72 & 6.64 & - & 6091.87 & B \\
\hline Fe I 6127.906 & 6.17 & $7.87 \mathrm{e}+05$ & 6127.85 & \\
\hline Fe I 6136.615 & 4.47 & $1.01 \mathrm{e}+06$ & 6136.62 & \\
\hline Fe I 6137.691 & 4.61 & $1.00 \mathrm{e}+06$ & 6137.64 & \\
\hline Fe I 6141.732 & 5.62 & $1.23 \mathrm{e}+06$ & 6141.66 & OJ \\
\hline Fe I 6157.728 & 6.09 & $1.20 \mathrm{e}+06$ & 6157.65 & \\
\hline Fe I 6165.36 & 6.15 & $6.54 \mathrm{e}+05$ & 6165.33 & W \\
\hline Fe I 6170.507 & 6.8 & - & 6170.51 & \\
\hline Fe I 6173.335 & 4.23 & $2.31 \mathrm{e}+05$ & 6173.32 & \\
\hline Fe I 6180.204 & 4.73 & $5.59 \mathrm{e}+04$ & 6180.17 & \\
\hline Fe I 6191.558 & 4.43 & $7.41 \mathrm{e}+05$ & 6191.52 & $\mathrm{P}$ \\
\hline Fe I 6200.312 & 4.61 & $9.06 e+04$ & 6200.26 & \\
\hline Fe I 6213.43 & 4.22 & $1.90 \mathrm{e}+05$ & 6213.4 & \\
\hline Fe I 6215.144 & 6.18 & $1.18 \mathrm{e}+06$ & 6215.16 & \\
\hline Fe I 6219.281 & 4.19 & $1.27 \mathrm{e}+05$ & 6219.27 & \\
\hline Fe I 6230.723 & 4.55 & $9.99 \mathrm{e}+05$ & 6230.72 & $\mathrm{~B}$ \\
\hline Fe I 6232.641 & 5.64 & $3.42 \mathrm{e}+06$ & 6232.54 & $\mathrm{~B}$ \\
\hline Fe I 6252.555 & 4.39 & $3.19 \mathrm{e}+05$ & 6252.54 & \\
\hline Fe I 6254.258 & 4.26 & $2.13 \mathrm{e}+05$ & 6254.3 & \\
\hline $\mathrm{Fe}$ I $6256.361 / \mathrm{Ni}$ I 6256.36 & $4.43 / 3.65$ & $7.40 \mathrm{e}+04 / 7.40 \mathrm{e}+04$ & 6256.3 & \\
\hline Fe I 6265.133 & 4.15 & $6.84 \mathrm{e}+04$ & 6265.09 & \\
\hline Fe I 6270.225 & 4.83 & $1.39 \mathrm{e}+05$ & 6270.27 & \\
\hline Fe I 6280.774 & 6.55 & - & 6280.79 & $\mathrm{~T}$ \\
\hline Fe I 6297.792 & 4.19 & $6.12 \mathrm{e}+04$ & 6297.78 & \\
\hline Fe I 6301.501 & 5.62 & $6.43 \mathrm{e}+06$ & 6301.47 & \\
\hline Fe I 6318.017/Ti I 6318.03 & $4.42 /-$ & $3.75 \mathrm{e}+05 /-$ & 6317.98 & \\
\hline Fe I 6322.685 & 4.55 & $6.95 \mathrm{e}+04$ & 6322.67 & \\
\hline Fe I 6335.331 & 4.15 & $1.58 \mathrm{e}+05$ & 6335.31 & \\
\hline Fe I 6336.824 & 5.64 & $7.71 \mathrm{e}+06$ & 6336.8 & B \\
\hline Fe I 6355.029 & 4.8 & $1.69 \mathrm{e}+05$ & 6355.03 & \\
\hline Fe I 6380.743 & 6.13 & $1.38 \mathrm{e}+06$ & 6380.71 & B \\
\hline Fe I 6393.601 & 4.37 & $4.81 \mathrm{e}+05$ & 6393.59 & $\mathrm{P}$ \\
\hline Fe I 6400.001 & 5.54 & $9.27 \mathrm{e}+06$ & 6400.01 & $\mathrm{P}$ \\
\hline Fe I 6408.018 & 5.62 & $3.12 \mathrm{e}+06$ & 6408.01 & \\
\hline Fe I 6411.649 & 5.59 & $7.86 \mathrm{e}+05$ & 6411.62 & \\
\hline
\end{tabular}


Table 3. continued.

\begin{tabular}{|c|c|c|c|c|}
\hline $\begin{array}{l}\text { Line } \lambda_{\text {lab }} \\
(\AA)\end{array}$ & $\begin{array}{c}E_{k} \\
(\mathrm{eV})\end{array}$ & $\begin{array}{c}A_{k i} \\
\left(\mathrm{~s}^{-1}\right) \\
\end{array}$ & $\begin{array}{l}\lambda_{\mathrm{obs}} \\
(\AA) \\
\end{array}$ & Comments \\
\hline Fe I 6416.930/Fe II 6416.905 & $6.73 / 5.82$ & $-1-$ & 6416.83 & \\
\hline Fe I 6421.351 & 4.21 & $3.04 \mathrm{e}+05$ & 6421.3 & \\
\hline Fe I 6462.725 & 2.83 & $5.60 \mathrm{e}+04$ & 6462.63 & $\mathrm{~B}$ \\
\hline Fe I 6475.624 & 4.47 & $2.60 \mathrm{e}+04$ & 6475.79 & $\mathrm{~W}$ \\
\hline Fe I 6481.87 & 4.19 & $3.29 \mathrm{e}+04$ & 6481.85 & \\
\hline Fe I 6494.98 & 4.31 & $7.66 \mathrm{e}+05$ & 6494.97 & \\
\hline Fe I 6498.939 & 2.87 & $4.64 \mathrm{e}+02$ & 6498.94 & \\
\hline Fe I 6546.237 & 4.65 & $9.06 \mathrm{e}+05$ & 6546.37 & \\
\hline Fe I 6592.914 & 4.61 & $7.37 \mathrm{e}+05$ & 6592.90 & \\
\hline Fe I 6593.87 & 4.31 & $5.28 \mathrm{e}+04$ & 6593.85 & \\
\hline Fe I 6604.588 & 6.71 & - & 6604.60 & \\
\hline Fe I 6609.11 & 4.43 & $3.45 \mathrm{e}+04$ & 6609.08 & \\
\hline Fe I 6663.442 & 4.28 & $4.98 \mathrm{e}+05$ & 6663.37 & \\
\hline Fe I 6703.567 & 4.61 & $1.8 \mathrm{e}+04$ & 6703.54 & \\
\hline Fe I 6750.152 & 4.26 & $1.17 \mathrm{e}+05$ & 6750.11 & \\
\hline Fe I 6769.659 & 6.41 & - & 6769.75 & M \\
\hline Fe I 6841.339 & 6.42 & $3.40 \mathrm{e}+06$ & 6841.27 & \\
\hline Fe I 6855.162 & 6.37 & $2.86 \mathrm{e}+06$ & 6855.15 & \\
\hline Fe I 6945.205 & 4.21 & $9.11 \mathrm{e}+04$ & 6945.17 & \\
\hline Fe I 6978.851 & 4.26 & $1.44 \mathrm{e}+05$ & 6978.81 & \\
\hline Fe I 6999.884 & 5.87 & $4.70 \mathrm{e}+05$ & 6999.83 & $\mathrm{~B}, \mathrm{~T}$ \\
\hline Fe I 7024.062 & 5.84 & $9.30 \mathrm{e}+04$ & 7024.0 & $\mathrm{~B}, \mathrm{~T}$ \\
\hline Fe I 7164.448 & 5.92 & - & 7164.52 & $\mathrm{~T}$ \\
\hline Fe I 7181.195 & 5.95 & - & 7181.19 & $\mathrm{~T}$ \\
\hline Fe I 7187.317 & 5.83 & $8.36 \mathrm{e}+06$ & 7187.21 & $\mathrm{~B}, \mathrm{~T}$ \\
\hline Fe I 7207.388 & 5.87 & - & 7207.36 & $\mathrm{~T}$ \\
\hline Fe I 7223.66 & 4.73 & $1.13 \mathrm{e}+05$ & 7223.57 & $\mathrm{~T}$ \\
\hline Fe I 7445.75 & 5.92 & - & 7445.73 & \\
\hline Fe I 7495.067 & 5.87 & - & 7495.01 & B \\
\hline Fe I 7511.02 & 5.83 & $1.35 \mathrm{e}+07$ & 7510.02 & \\
\hline Fe I 7531.145 & 6.02 & - & 7531.12 & M \\
\hline Fe I 7588.305 & 6.67 & - & 7588.33 & \\
\hline Fe I 7748.269 & 4.55 & $2.19 \mathrm{e}+05$ & 7748.2 & \\
\hline Fe I 7788.742 & 5.78 & - & 7788.78 & \\
\hline Fe I 7832.196 & 6.02 & - & 7832.18 & \\
\hline Fe I 7912.867 & 2.43 & $1.68 \mathrm{e}+02$ & 7912.81 & \\
\hline Fe I 7937.14 & 5.87 & - & 7937.12 & \\
\hline Fe I 7945.846 & 5.95 & - & 7945.79 & \\
\hline Fe I 7998.945 & 5.92 & - & 7999.04 & \\
\hline Fe I 8027.941 & 4.8 & - & 8027.99 & \\
\hline Fe I 8046.047 & 5.96 & - & 8046.06 & \\
\hline Fe I 8047.618 & 2.4 & - & 8047.64 & \\
\hline Fe I 8085.172 & 5.98 & - & 8085.13 & \\
\hline Fe I 8220.378 & 5.83 & $1.69 \mathrm{e}+07$ & 8220.35 & \\
\hline Fe I 8327.056 & 3.69 & $9.57 \mathrm{e}+05$ & 8327.01 & \\
\hline Fe I 8331.915 & 5.87 & - & 8331.98 & \\
\hline Fe I 8387.771 & 3.65 & $6.09 \mathrm{e}+05$ & 8387.72 & \\
\hline Fe I 8582.257 & 4.43 & $7.40 \mathrm{e}+04$ & 8582.17 & \\
\hline Fe I 8611.804 & 4.28 & $1.27 \mathrm{e}+06$ & 8611.76 & \\
\hline Fe I 8688.625 & 3.6 & $7.74 e+05$ & 8688.48 & \\
\hline Fe I 8712.885 & 7.11 & - & 8712.83 & $\mathrm{~W}$ \\
\hline
\end{tabular}


Table 3. continued.

\begin{tabular}{|c|c|c|c|c|}
\hline $\begin{array}{l}\text { Line } \lambda_{\text {lab }} \\
(\AA)\end{array}$ & $\begin{array}{c}E_{k} \\
(\mathrm{eV})\end{array}$ & $\begin{array}{c}A_{k i} \\
\left(\mathrm{~s}^{-1}\right)\end{array}$ & $\begin{array}{l}\lambda_{\mathrm{obs}} \\
(\AA)\end{array}$ & Comments \\
\hline Fe I 8751.026 & 7.36 & - & 8751.1 & \\
\hline Fe I 8824.221 & 3.6 & $3.53 e+05$ & 8824.1 & P, S, Wi-I \\
\hline Fe I 8838.299/.429 & $7.22 / 4.26$ & $-/ 3.83 e+05$ & 8838.37 & \\
\hline Fe I 8999.556 & 4.21 & $1.06 \mathrm{e}+06$ & 8999.54 & $\mathrm{~T}$ \\
\hline Fe II 3824.929 & 5.82 & $3.20 \mathrm{e}+03$ & 3824.97 & \\
\hline Fe II 4122.668/Fe I 4122.515 & $5.59 / 5.85$ & $3.30 \mathrm{e}+04 / 3.23 \mathrm{e}+06$ & 4122.58 & \\
\hline Fe II 4128.748 & 5.58 & $2.60 \mathrm{e}+04$ & 4128.67 & \\
\hline Fe II 4173.461 & 5.55 & $4.43 e+05$ & 4173.44 & \\
\hline Fe II 4178.862 & 5.55 & $1.72 \mathrm{e}+05$ & 4178.82 & \\
\hline Fe II 4233.167 & 5.51 & $7.22 \mathrm{e}+05$ & 4233.15 & $\mathrm{P}$ \\
\hline Fe II 4258.154 & 5.62 & $3.10 \mathrm{e}+04$ & 4258.18 & \\
\hline Fe II 4273.326 & 5.6 & $9.10 \mathrm{e}+04$ & 4273.26 & Wer \\
\hline Fe II 4296.572 & 5.59 & $7.00 \mathrm{e}+04$ & 4296.54 & \\
\hline Fe II 4303.176 & 5.58 & $2.20 \mathrm{e}+05$ & 4303.16 & \\
\hline Fe II 4351.769 & 5.55 & $4.86 \mathrm{e}+05$ & 4351.75 & $\mathrm{P}$ \\
\hline 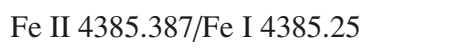 & $5.60 / 5.84$ & $4.50 \mathrm{e}+05 /-$ & 4385.34 & \\
\hline Fe II 4416.83 & 5.58 & $2.10 \mathrm{e}+05$ & 4416.79 & \\
\hline Fe II 4489.183 & 5.59 & $5.90 \mathrm{e}+04$ & 4489.17 & \\
\hline Fe II 4491.405 & 5.16 & $1.89 \mathrm{e}+05$ & 4491.36 & $\mathrm{~B}$ \\
\hline Fe II 4508.288 & 5.6 & $7.30 \mathrm{e}+05$ & 4508.26 & \\
\hline Fe II 4515.339 & 5.59 & $2.37 \mathrm{e}+05$ & 4515.3 & \\
\hline Fe II 4520.224 & 5.55 & $9.80 \mathrm{e}+04$ & 4520.19 & \\
\hline Fe II 4522.632/.634 & 5.58 & $-/ 8.40 \mathrm{e}+05$ & 4522.77 & B, Ser \\
\hline Fe II 4549.474 & 5.55 & $1.00 \mathrm{e}+06$ & 4549.45 & $\mathrm{P}$ \\
\hline Fe II 4555.893 & 5.55 & $2.26 \mathrm{e}+05$ & 4555.85 & \\
\hline Fe II 4576.34 & 5.55 & $6.40 \mathrm{e}+04$ & 4576.31 & \\
\hline Fe II 4583.829 & 5.51 & $7.22 \mathrm{e}+05$ & 4583.81 & \\
\hline Fe II 4629.339 & 5.48 & $1.72 \mathrm{e}+05$ & 4629.44 & \\
\hline Fe II 4656.981 & 5.55 & $1.37 \mathrm{e}+04$ & 4657.04 & \\
\hline Fe II 4666.758 & 5.48 & $1.30 \mathrm{e}+04$ & 4666.76 & \\
\hline Fe II 4670.182 & 5.24 & $3.20 \mathrm{e}+03$ & 4670.05 & B, Wer \\
\hline Fe II 4731.453 & 5.51 & $2.80 \mathrm{e}+04$ & 4731.43 & \\
\hline Fe II 4833.197 & 5.22 & $4.60 \mathrm{e}+02$ & 4833.02 & W \\
\hline Fe II 4839.998 & 5.24 & $4.00 \mathrm{e}+02$ & 4839.93 & $\mathrm{~W}$ \\
\hline Fe II 4923.921 & 5.41 & $4.28 \mathrm{e}+06$ & 4923.9 & Wi \\
\hline Fe II 4993.358 & 5.29 & $6.90 \mathrm{e}+03$ & 4993.37 & W \\
\hline Fe II 5018.434 & 5.36 & $2.0 \mathrm{e}+06$ & 5018.42 & Wi \\
\hline Fe II 5169.03 & 5.29 & $4.22 \mathrm{e}+06$ & 5169.0 & \\
\hline Fe II 5197.577 & 5.62 & $5.40 \mathrm{e}+05$ & 5197.53 & \\
\hline Fe II 5234.625 & 5.59 & $2.50 \mathrm{e}+05$ & 5234.58 & $\mathrm{P}$ \\
\hline Fe II 5264.176/Mg II 5264.215/.22 & $12.86 / 13.92$ & $4.76 \mathrm{e}+07 / 1.27 \mathrm{e}+07$ & 5264.19 & B, Wer \\
\hline Fe II 5264.812 & 5.58 & $3.52 \mathrm{e}+04$ & 5264.82 & $\mathrm{~W}, \mathrm{~B}$ \\
\hline Fe II 5276.002 & 5.55 & $3.76 \mathrm{e}+05$ & 5275.97 & $\mathrm{P}$ \\
\hline Fe II 5284.109 & 5.24 & $1.90 \mathrm{e}+04$ & 5284.07 & \\
\hline Fe II 5316.615 & 5.48 & $3.89 \mathrm{e}+05$ & 5316.6 & P, Wi-I \\
\hline Fe II 5325.553 & 5.55 & $8.00 \mathrm{e}+04$ & 5325.55 & WJ \\
\hline Fe II 5362.86 & - & - & 5362.84 & S, Wi, (AJJ86) \\
\hline Fe II 5534.847 & 5.48 & $3.00 \mathrm{e}+04$ & 5534.8 & $\mathrm{P}$ \\
\hline Fe II 5627.497 & 5.59 & $2.93 e+03$ & 5627.5 & $\mathrm{~W}$ \\
\hline Fe II 5813.677 & 7.7 & $8.80 \mathrm{e}+04$ & 5813.65 & W \\
\hline
\end{tabular}


Table 3. continued.

\begin{tabular}{|c|c|c|c|c|}
\hline $\begin{array}{l}\text { Line } \lambda_{\text {lab }} \\
(\AA)\end{array}$ & $\begin{array}{c}E_{k} \\
(\mathrm{eV})\end{array}$ & $\begin{array}{c}A_{k i} \\
\left(\mathrm{~s}^{-1}\right)\end{array}$ & $\begin{array}{l}\lambda_{\mathrm{obs}} \\
(\AA)\end{array}$ & Comments \\
\hline Fe II 5823.155 & 7.7 & $2.00 \mathrm{e}+04$ & 5823.06 & $\mathrm{~W}$ \\
\hline Fe II 5991.376 & 5.22 & $4.20 \mathrm{e}+03$ & 5991.36 & $\mathrm{P}$ \\
\hline Fe II 6084.111 & 5.24 & $3.00 \mathrm{e}+03$ & 6084.05 & $\mathrm{P}$ \\
\hline Fe II 6113.322 & 5.25 & $1.70 \mathrm{e}+03$ & 6113.23 & W \\
\hline Fe II 6147.735 & 5.9 & $1.3 e+05$ & 6147.76 & $\mathrm{~B}$ \\
\hline Fe II 6149.238 & 5.9 & $1.3 e+05$ & 6149.19 & B \\
\hline Fe II 6175.14 & 8.23 & $1.8 \mathrm{e}+05$ & 6175.11 & B \\
\hline Fe II 6238.375 & 5.88 & $7.5 e+04$ & 6238.38 & $\mathrm{P}$ \\
\hline Fe II 6247.562 & 5.87 & $1.6 e+05$ & 6247.5 & $\mathrm{P}$ \\
\hline Fe II 6432.68 & 4.82 & $8.50 \mathrm{e}+03$ & 6432.63 & $\mathrm{P}$ \\
\hline Fe II 6456.376 & 5.82 & $1.7 \mathrm{e}+05$ & 6456.33 & $\mathrm{P}$ \\
\hline Fe II 6516.053 & 4.79 & $8.3 e+03$ & 6516.02 & $\mathrm{P}$ \\
\hline Fe II 7462.38 & 5.55 & $2.7 e+04$ & 7462.34 & $\mathrm{P}$ \\
\hline Fe II 7479.694 & 5.55 & $3.50 \mathrm{e}+03$ & 7479.46 & W, B \\
\hline Fe II 7515.832 & 5.55 & $8.10 \mathrm{e}+03$ & 77515.46 & B \\
\hline Fe II 7711.71 & 5.51 & $4.94 \mathrm{e}+04$ & 7711.79 & $\mathrm{P}$ \\
\hline Co I 3745.50 & 4.23 & $7.5 e+06$ & 3745.42 & \\
\hline Co I 3894.08 & 4.23 & $6.9 \mathrm{e}+07$ & 3894.17 & \\
\hline Co I 3922.752/Fe I 3922.912 & $4.21 / 3.21$ & $5.70 \mathrm{e}+05 / 1.08 \mathrm{e}+06$ & 3922.84 & \\
\hline Co I 4110.54 & 4.06 & $5.5 \mathrm{e}+06$ & 4110.56 & \\
\hline Co I 4118.77 & 4.06 & $1.6 \mathrm{e}+07$ & 4118.79 & \\
\hline Co I 4132.144 & 4.05 & $9.90 \mathrm{e}+04$ & 4132.2 & \\
\hline Co I 5146.161 & 4.15 & $9.20 \mathrm{e}+03$ & 5146.18 & \\
\hline Co I 7016.62 & 3.77 & $4.60 \mathrm{e}+05$ & 7016.5 & $\mathrm{~T}$ \\
\hline Co I 7154.67 & 3.77 & $1.20 \mathrm{e}+05$ & 7154.89 & B: \\
\hline Ni I 3775.57 & 3.71 & $4.20 \mathrm{e}+06$ & 3775.56 & $\mathrm{~B}$ \\
\hline Ni I 3807.14 & 3.68 & $4.30 \mathrm{e}+06$ & 3807.09 & $\mathrm{P}$ \\
\hline Ni I 3831.69 & 3.66 & $1.50 \mathrm{e}+06$ & 3831.71 & \\
\hline Ni I 4648.66 & 6.09 & $2.4 \mathrm{e}+07$ & 4648.73 & M \\
\hline Ni I 4756.52 & 6.09 & $1.5 \mathrm{e}+07$ & 4756.5 & \\
\hline Ni I 4763.95 & - & - & 4763.88 & $\mathrm{P}$ \\
\hline Ni I 4790.965 & 4.54 & $2.3 e+04$ & 4791.06 & \\
\hline Ni I 5137.08 & 4.09 & $8.6 e+05$ & 5137.13 & \\
\hline Ni I 5142.77 & - & - & 5142.79 & \\
\hline Ni I 5265.712 & 6.01 & $3.70 \mathrm{e}+05$ & 5265.68 & \\
\hline Ni I 5476.91 & 4.09 & $9.5 \mathrm{e}+06$ & 5476.85 & \\
\hline Ni I 5578.73 & 3.9 & $9.8 \mathrm{e}+04$ & 5578.7 & \\
\hline Ni I 5587.86 & 4.15 & $2.2 \mathrm{e}+05$ & 5587.83 & \\
\hline Ni I 5754.68 & 4.09 & $3.1 \mathrm{e}+05$ & 5754.64 & $\mathrm{~S} / \mathrm{N}$ \\
\hline Ni I 6108.12 & 3.71 & $1.3 e+05$ & 6108.09 & \\
\hline Ni I 6643.64 & 3.54 & $1.5 \mathrm{e}+05$ & 6643.59 & \\
\hline Ni I 6767.77 & 3.66 & $3.3 e+05$ & 6767.73 & $\mathrm{~B}$ \\
\hline Ni I 7989.945 & 1.83 & - & 7989.75 & W \\
\hline INDEF 3927.1 & - & - & 3927.1 & OA, Fe I 3927.74, .61? \\
\hline INDEF 4048.47 & - & - & 4048.47 & Mn I $4048.76 ?$ \\
\hline INDEF 4086.3 & - & - & 4086.3 & \\
\hline INDEF 4159.17 & - & - & 4159.17 & \\
\hline INDEF 4410.64 & - & - & 4410.64 & B, Mn I 4410.50?, Ni I 4410.52? \\
\hline
\end{tabular}


Table 3. continued.

\begin{tabular}{|c|c|c|c|c|}
\hline $\begin{array}{l}\text { Line } \lambda_{\text {lab }} \\
(\AA)\end{array}$ & $\begin{array}{c}E_{k} \\
(\mathrm{eV})\end{array}$ & $\begin{array}{c}A_{k i} \\
\left(\mathrm{~s}^{-1}\right)\end{array}$ & $\begin{array}{l}\lambda_{\mathrm{obs}} \\
(\AA)\end{array}$ & Comments \\
\hline INDEF 4421.97 & - & - & 4421.97 & $\mathrm{~W}$ \\
\hline INDEF 4787.18 & - & - & 4787.18 & $\mathrm{~S} / \mathrm{N}$ \\
\hline INDEF 5109.19 & - & - & 5109.19 & M, Sc I 5109.07? \\
\hline INDEF 5598.33 & - & - & 5598.33 & \\
\hline INDEF 5684.23 & - & - & 5684.23 & Wi, Sc II 5684.20?, Fe I 5684.48?, Si I 5684.48? \\
\hline INDEF 5756.39 & - & - & 5756.39 & $\mathrm{M}, \mathrm{S} / \mathrm{N}$ \\
\hline INDEF 5792.69 & - & - & 5792.69 & \\
\hline INDEF 5798.77 & - & - & 5798.77 & $\mathrm{~S} / \mathrm{N}$ \\
\hline INDEF 6010.27 & - & - & 6010.27 & $\mathrm{~S} / \mathrm{N}$ \\
\hline INDEF 6023.6 & - & - & 6023.6 & $\mathrm{NJ}$ \\
\hline INDEF 6171.51 & - & - & 6171.51 & $\mathrm{~W}, \mathrm{M}$ \\
\hline INDEF 6263.72 & - & - & 6263.72 & M \\
\hline INDEF 6266.95 & - & - & 6266.95 & \\
\hline INDEF 6741.84 & - & - & 6741.84 & Si I 6741.64? T: \\
\hline INDEF 6795.59 & - & - & 6795.59 & $\mathrm{~B}, \mathrm{~S} / \mathrm{N}$ \\
\hline INDEF 6828.33 & - & - & 6828.33 & \\
\hline INDEF 7291.23 & - & - & 7291.23 & T, Mg I 7291.06?, Ni I 7291.45? \\
\hline INDEF 7355.57 & - & - & 7355.57 & $\mathrm{~S}$ \\
\hline INDEF 7513.32 & - & - & 7513.32 & B, Ba I $7513.46 ?$ \\
\hline INDEF 7515.46 & - & - & 7515.46 & Fe II $7515.83 ?$ \\
\hline INDEF 7533.12 & - & - & 7533.12 & \\
\hline INDEF 7748.48 & - & - & 7748.48 & \\
\hline INDEF 7841.79 & - & - & 7841.79 & \\
\hline INDEF 7896.58 & - & - & 7896.58 & P, Mg II 7896.04? \\
\hline INDEF 7914.15 & - & - & 7914.15 & \\
\hline INDEF 8048.74 & - & - & 8048.74 & Fe I $8048.99 ?$ \\
\hline INDEF 8345.05 & - & - & 8345.05 & \\
\hline INDEF 8380.41 & - & - & 8380.41 & W, Sc II 8380.60? \\
\hline INDEF 8389.15 & - & - & 8389.15 & S, OA, Fe I 8387.77? \\
\hline
\end{tabular}




\section{Appendix A: Gaussian fits to the emission lines in outburst}

We describe here in detail the three Gaussian fits to the independent lines in the outburst, and present a few more examples of the resulting best fits to some of the metallic lines (see Figs. A.1 to A.5). The fits were done with IRAF task ngaussfit, restricting the fitting range to $\pm 250 \mathrm{~km} \mathrm{~s}^{-1}$ (or less, in the case of proximity to another feature). The fitting was done interactively, paying special attention to the continuum and the presence of the NC. The model fitted to the data can be written as

$F_{\text {total }}(v)=a+b v+\sum_{i=1}^{3} A_{i} \mathrm{e}^{-\frac{\left(v-C_{i}\right)^{2}}{2\left(F W H M_{i} / 2 \sqrt{2 \ln 2)^{2}}\right.}}$,

where $a$ and $b$ are the continuum level and slope, $A_{i}$ are the amplitudes of the different Gaussian components, $C_{i}$ are the Gaussian centers (in velocity), and $F W H M_{i}$ are the FWHM of the three Gaussian components. The full collection of best fits to the lines unaffected by blending is shown in Table A.1. While the $\mathrm{BC}$ of neutral and ionized metallic lines observed in May
2008 are strongly consistent with two broad Gaussian components with variable velocities, the April and June observations in 2008 were not so uniquely defined. Fitting in terms of a single broad component plus either a narrower blueshifted or redshifted absorption component typically provides the best fits to the metallic lines (and would be a signature of wind and infall, respectively), but we cannot a priori rule out an interpretation in terms of two Gaussian emission components. In several cases during these two dates, a simple three Gaussian fit to all the components in emission fails to reproduce the blueshifted and redshifted absorption profiles observed, which reinforces the interpretation of the line profiles in terms of wind- and infallrelated absorption. In addition, in some other cases, the fits are not unique (e.g. Fe II line on April 21, see Table A.1).

On the other hand, the $\mathrm{H}$ I and He I lines appear much more symmetric than the metallic lines at all dates, and the asymmetries that could be potentially interpreted as independent components do not show any clear dynamical pattern in the different observations, which clearly distinguishes them from the metallic lines.

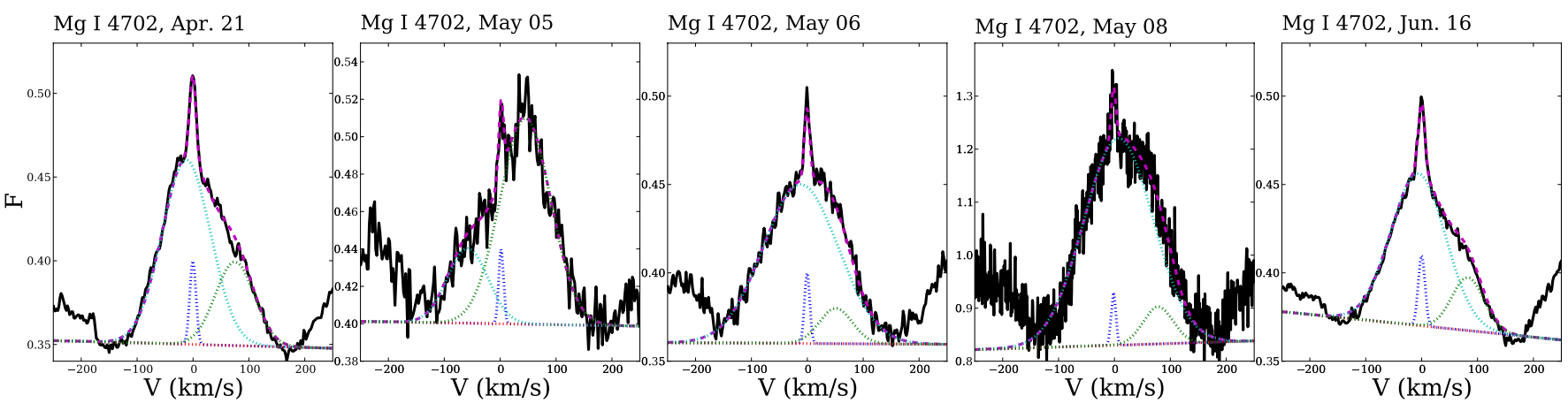

Fig. A.1. Mg I $4702 \AA$ A line: results of the fit with three Gaussian components for the different outburst epochs. The original data is shown as the bold line, individual Gaussians are marked by dotted lines, and the final fit is shown as a dashed line.
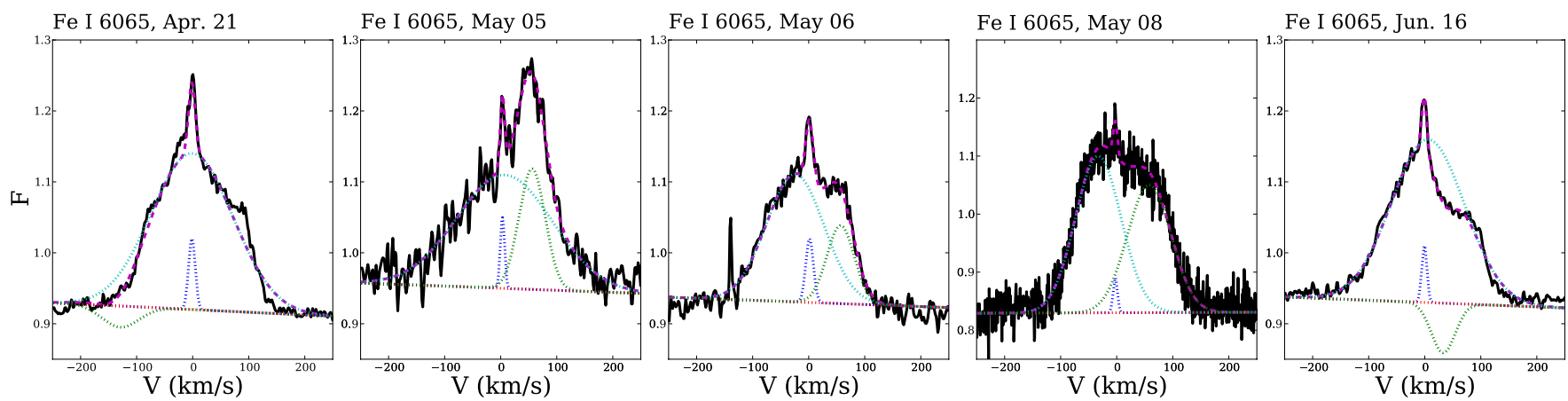

Fig. A.2. Fe I $6065 \AA$ line: results of the fit with three Gaussian components for the different outburst epochs. The original data is shown as the bold line, individual Gaussians are marked by dotted lines, and the final fit is shown as a dashed line. 


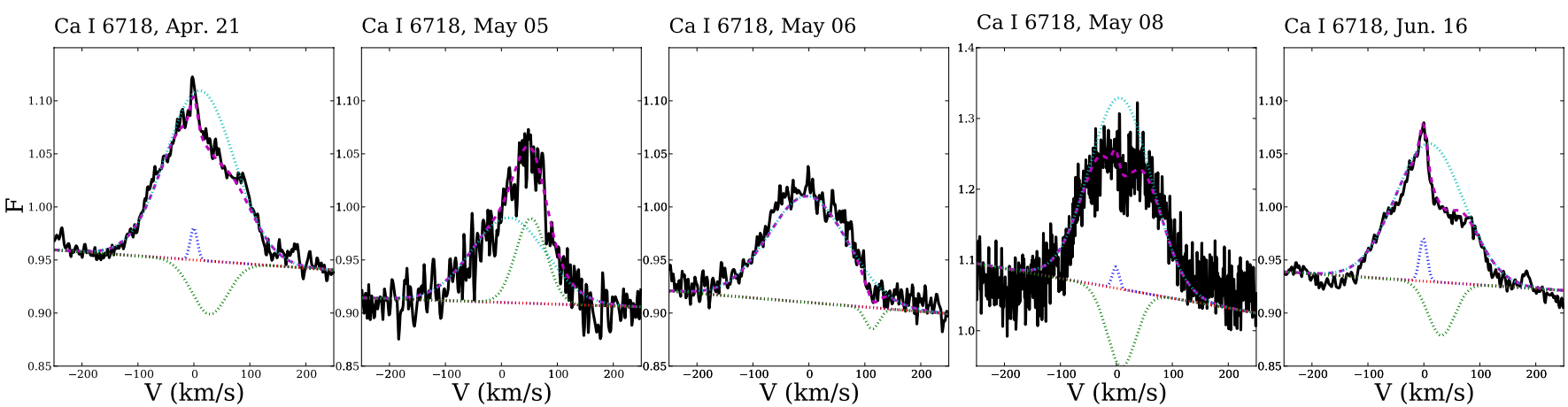

Fig. A.3. Ca I 6718 A line: results of the fit with three Gaussian components for the different outburst epochs. The original data is shown as the bold line, individual Gaussians are marked by dotted lines, and the final fit is shown as a dashed line.

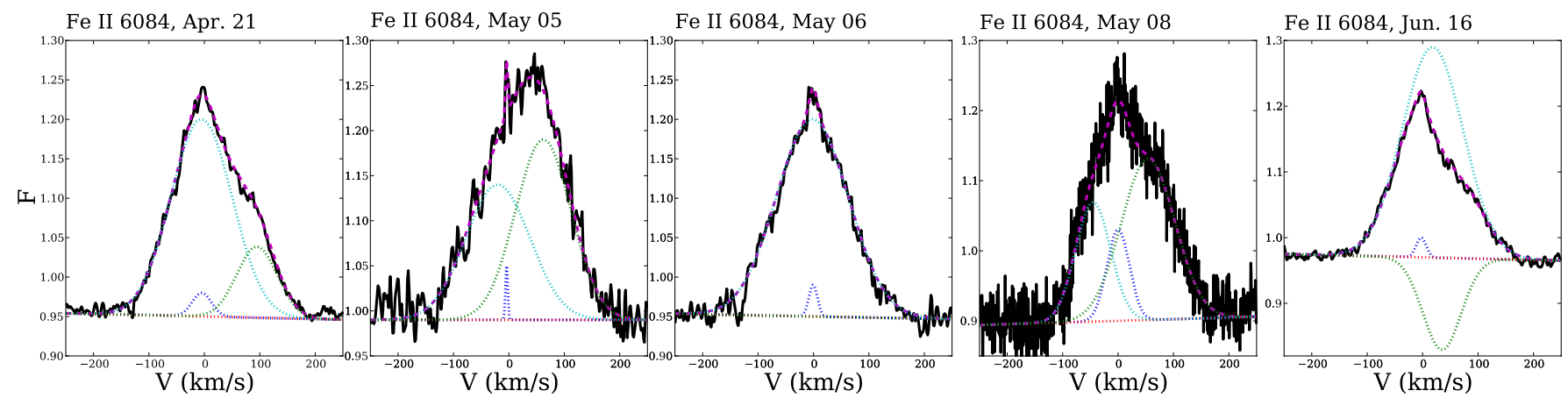

Fig. A.4. Fe II 6084 A line: results of the fit with three Gaussian components for the different outburst epochs. The original data is shown as the bold line, individual Gaussians are marked by dotted lines, and the final fit is shown as a dashed line.
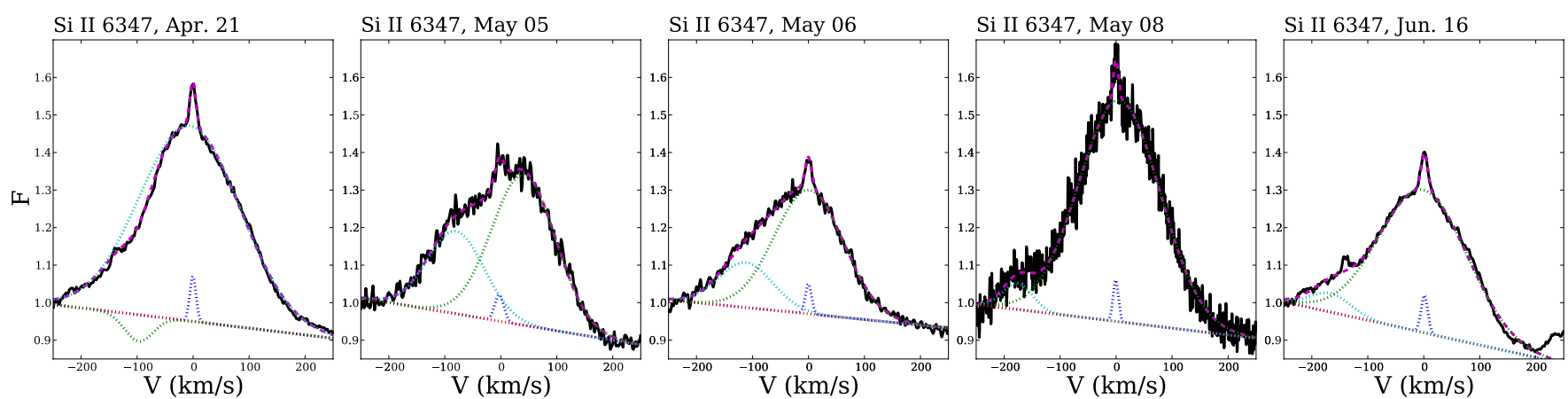

Fig. A.5. Si II $6347 \AA$ A line: results of the fit with three Gaussian components for the different outburst epochs. The original data is shown as the bold line, individual Gaussians are marked by dotted lines, and the final fit is shown as a dashed line. Note that the blueshifted emission that appears on June 16 is probably due to contamination by a weaker, blended line that became visible as the continuum and BC strength decreased. 


\begin{tabular}{|c|c|c|c|}
\hline & 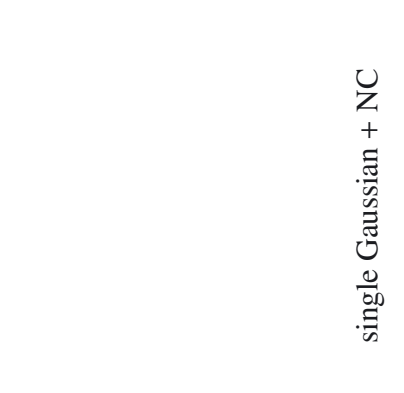 & 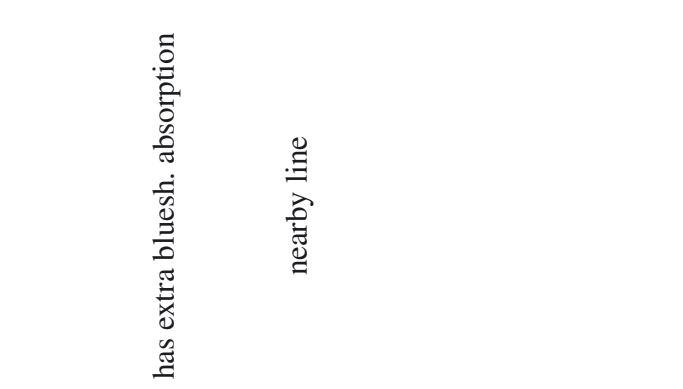 & $U$ \\
\hline$\sum_{1}^{2}$ & 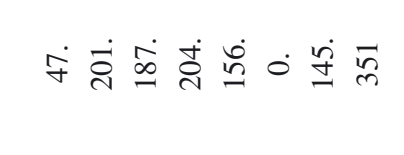 & 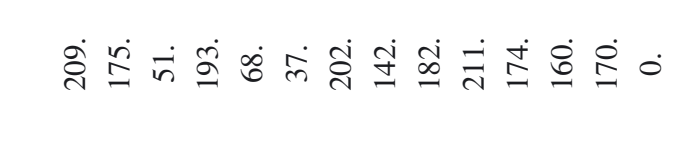 & 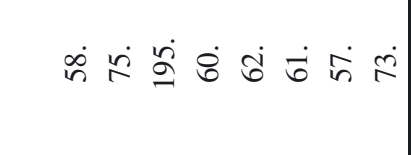 \\
\hline$\tilde{U}$ & 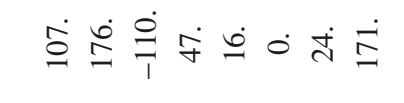 & 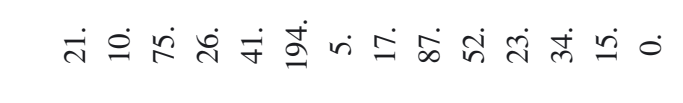 & 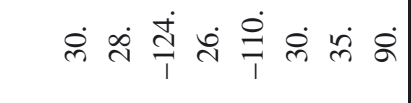 \\
\hline$m$ & 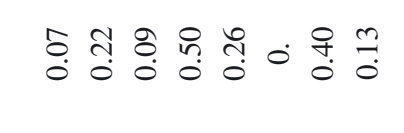 & 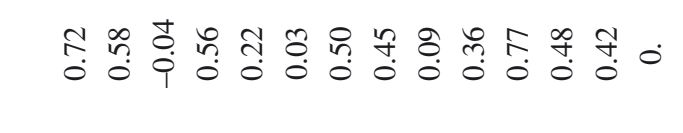 & 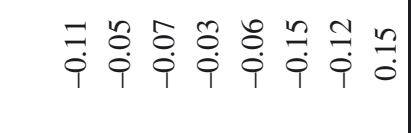 \\
\hline 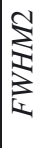 & 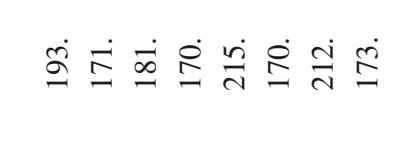 & 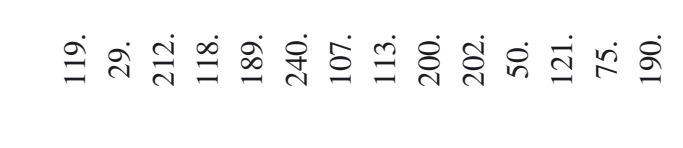 & 守守 ウ்ं \\
\hline $\mathcal{U}$ & 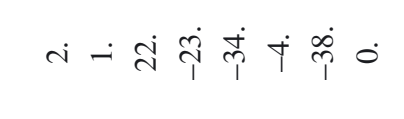 & 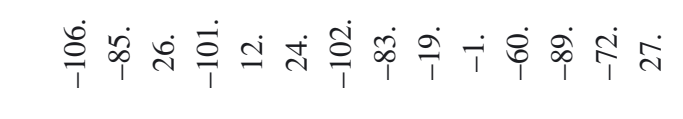 & 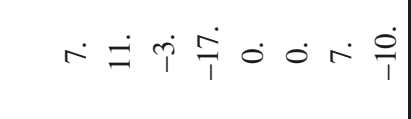 \\
\hline $\mathcal{z}$ & શิ & 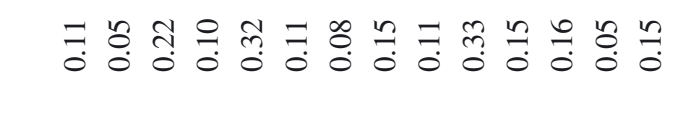 & 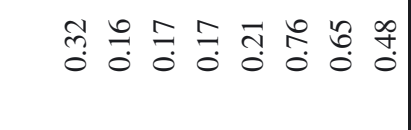 \\
\hline 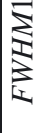 & $\dot{F} \dot{\circ} \dot{f} \dot{t} \dot{0} \dot{\circ} \dot{F}$ & 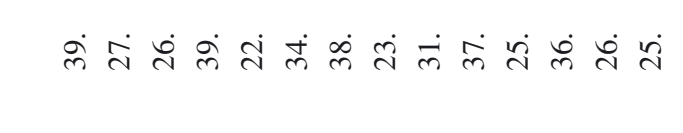 & 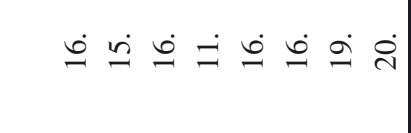 \\
\hline $\bar{u}$ & 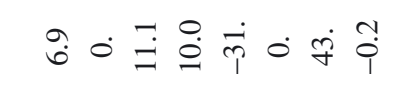 & 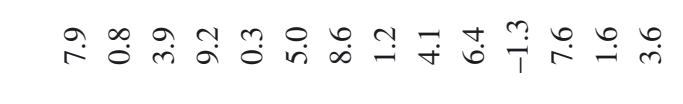 & 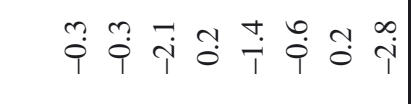 \\
\hline $\bar{F}$ & 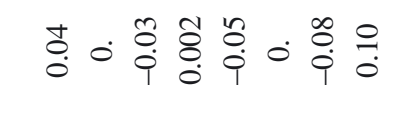 & సֶ. & 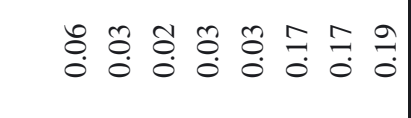 \\
\hline 0 & 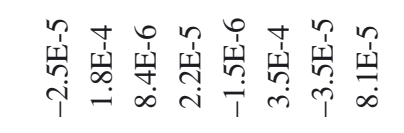 & 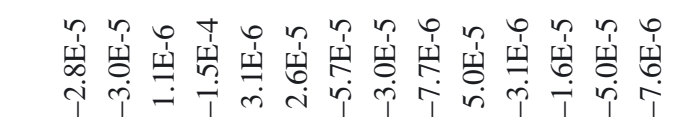 & 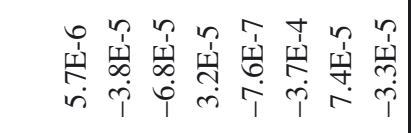 \\
\hline 0 & 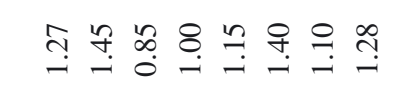 & 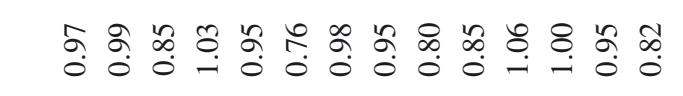 & 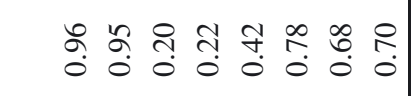 \\
\hline$\tilde{\Xi}$ & 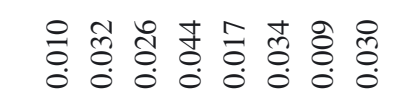 & 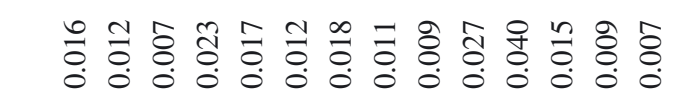 & 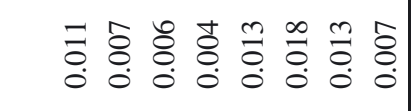 \\
\hline 7 & 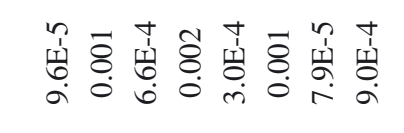 & 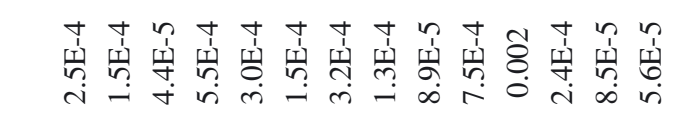 & 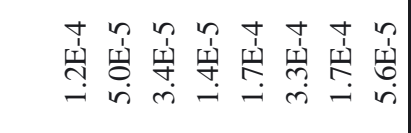 \\
\hline $\begin{array}{l}\stackrel{0}{\tilde{N}} \\
\end{array}$ & 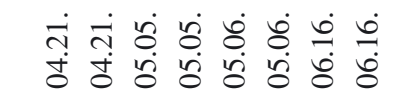 & 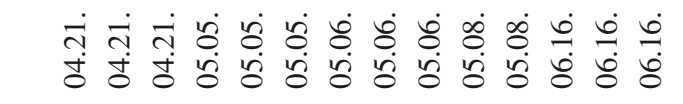 & 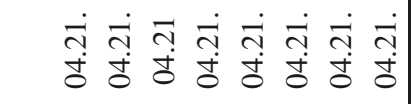 \\
\hline & 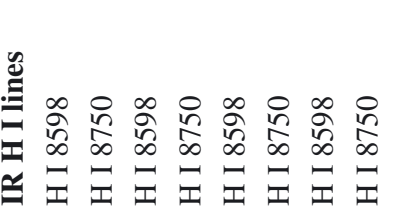 & 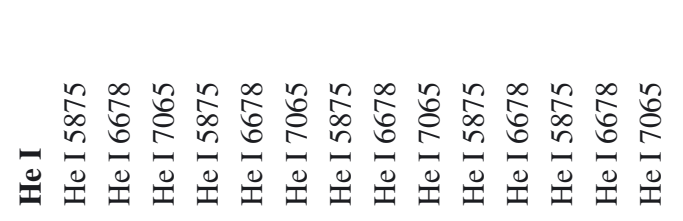 & 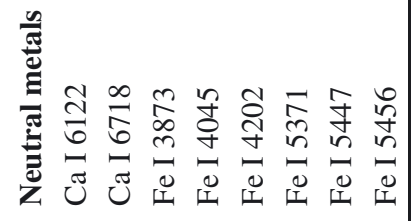 \\
\hline
\end{tabular}




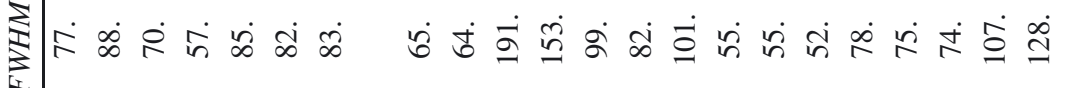
U

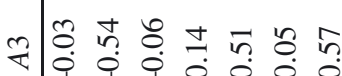
2

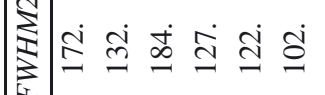

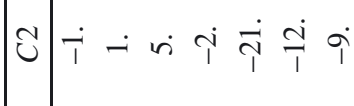

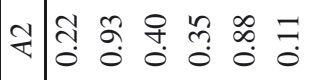
$\Xi$

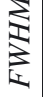
U

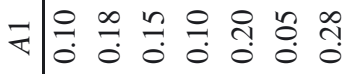

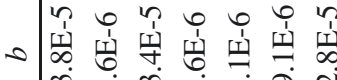
- 2 :

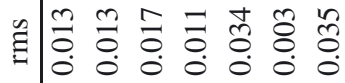

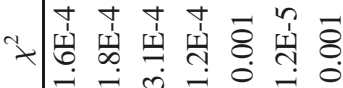

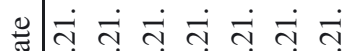

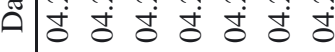

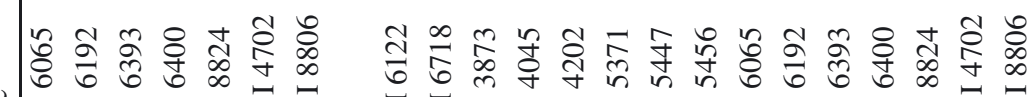

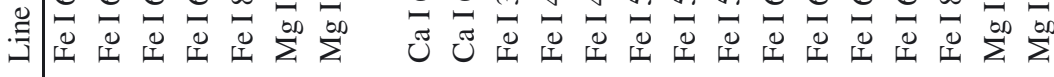

லூ่

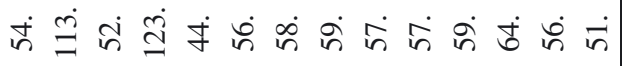
궁 ఏேீல் तें 앙 융

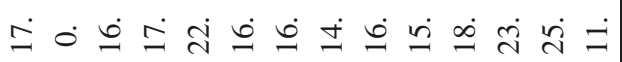

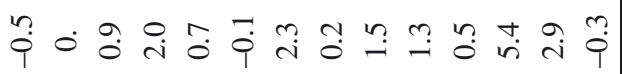

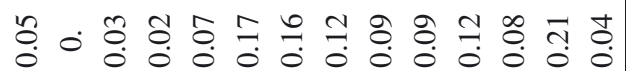
rin o n n t n n n n n o o o

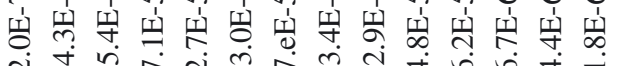
तर

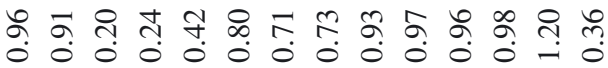

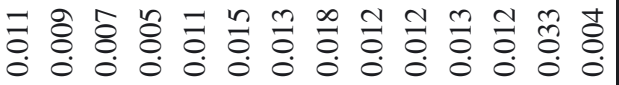

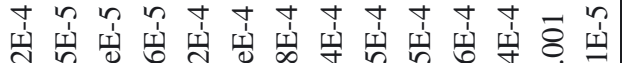
กำ

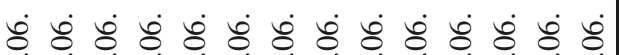

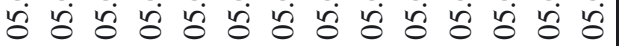

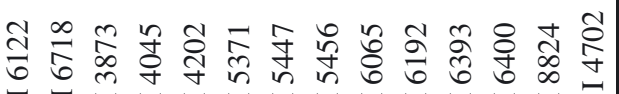

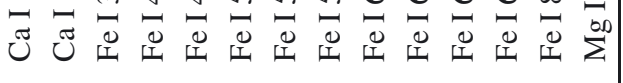




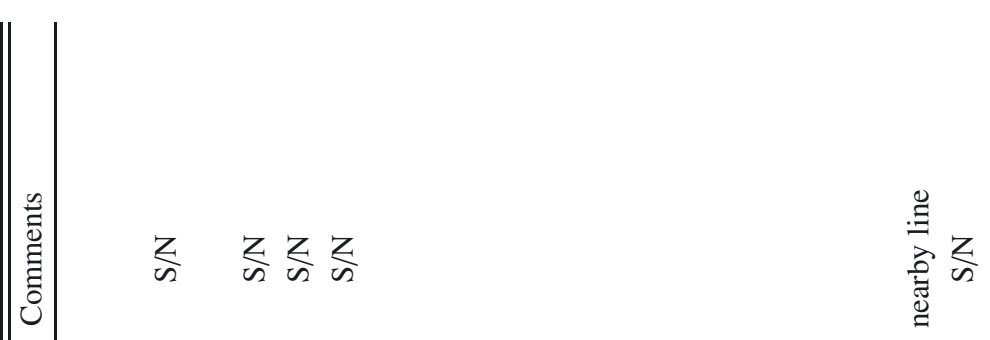

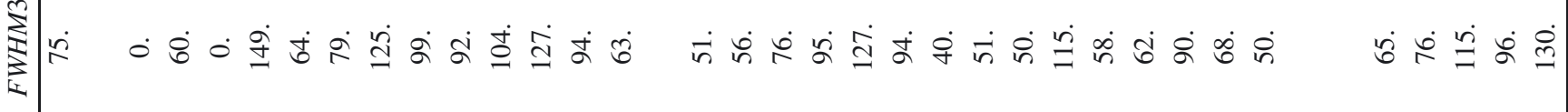

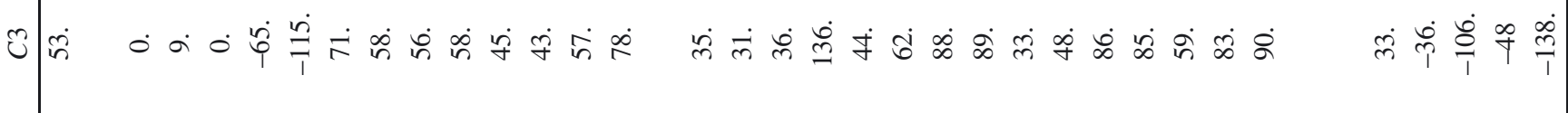

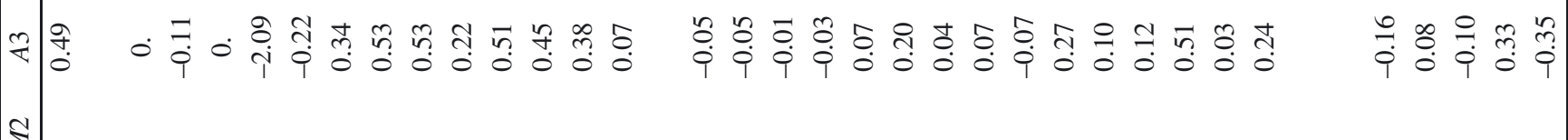
这

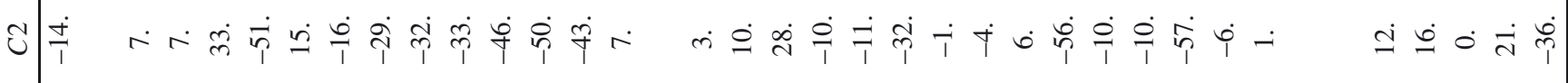

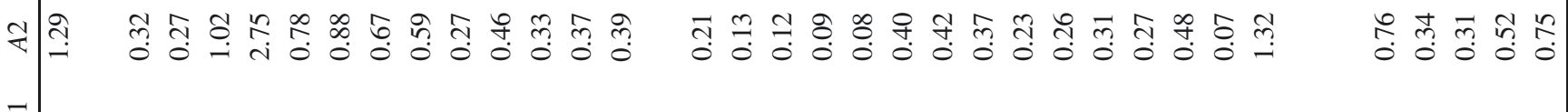
थि के

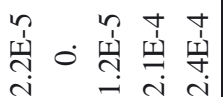
•

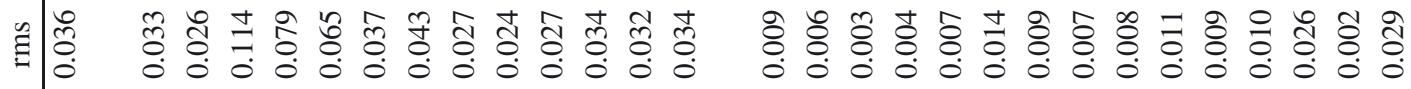

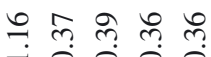

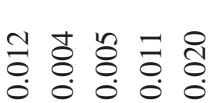

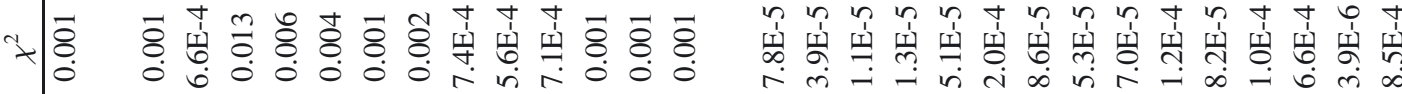

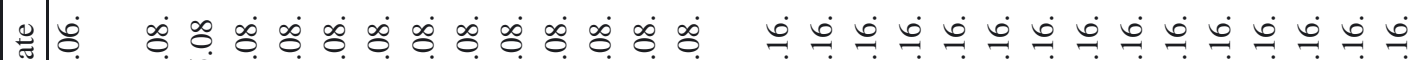
ச் ச் ச் ம் 要

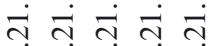
过过莳

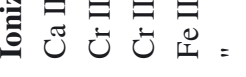




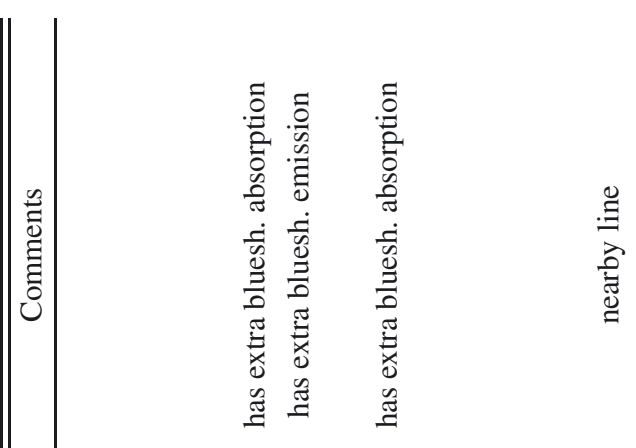

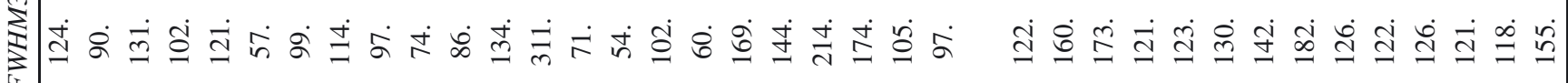

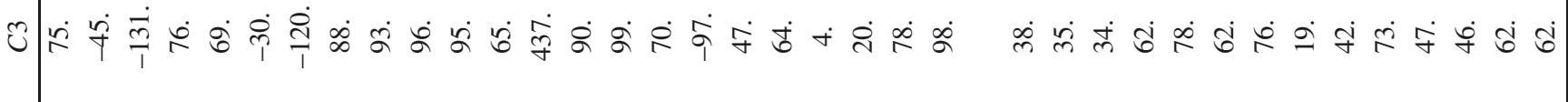
ॠ

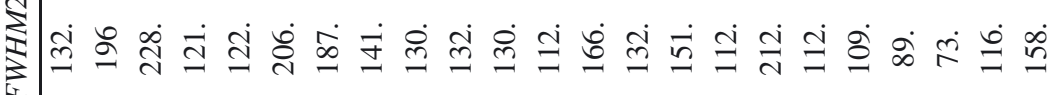
வூં

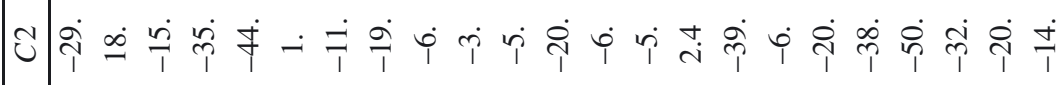
장ํㅇ

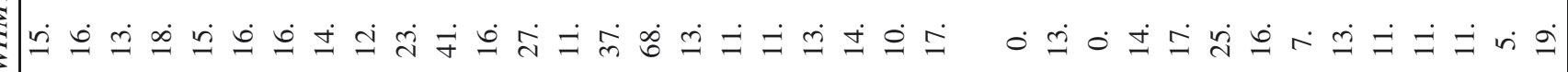

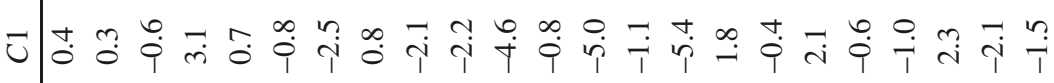

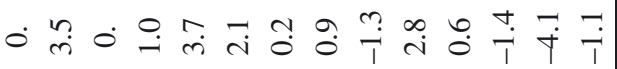

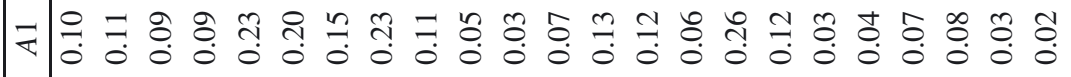

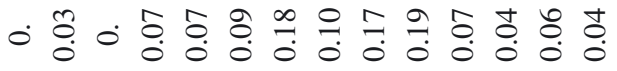

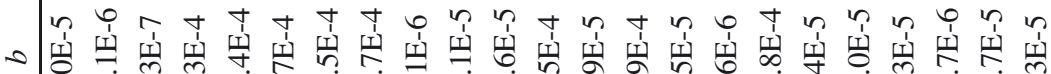
光

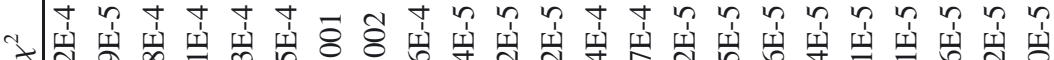

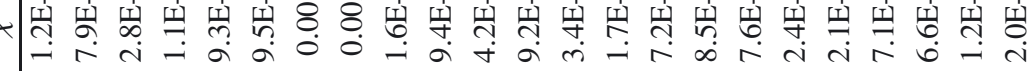

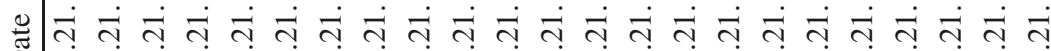

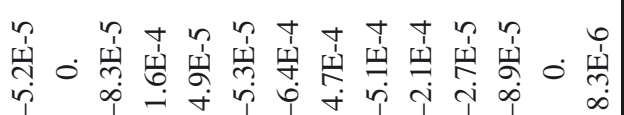

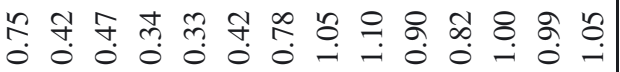

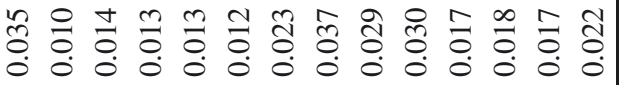

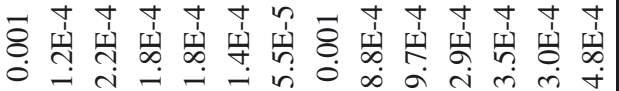

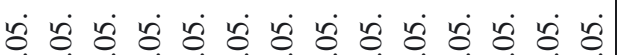

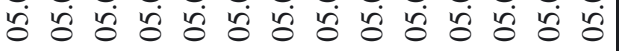

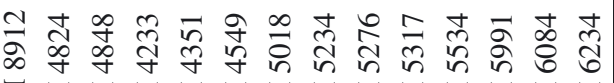

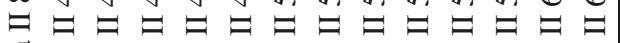

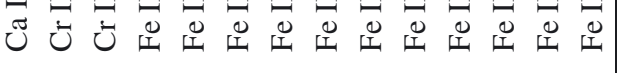




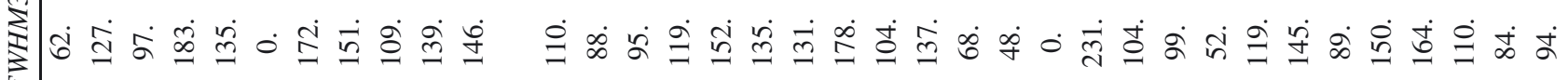

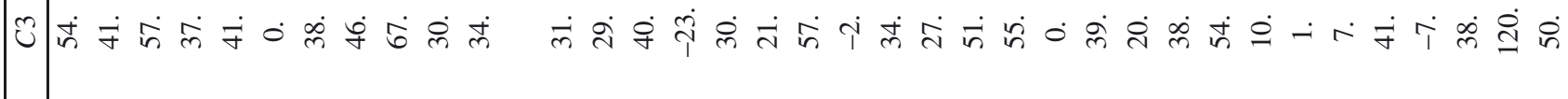

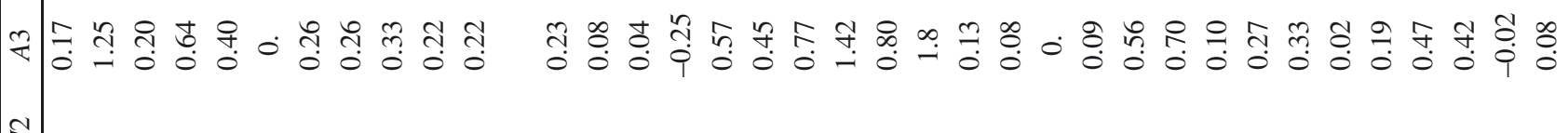

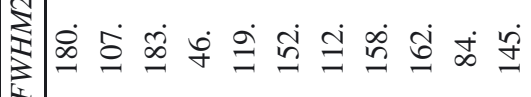

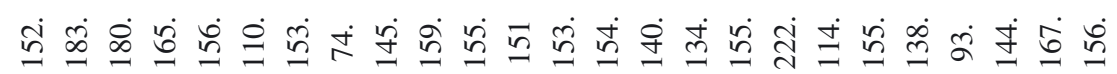
U人i

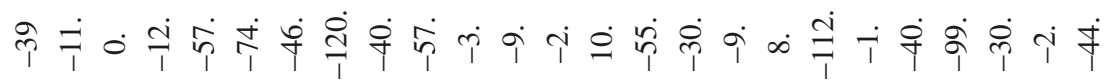

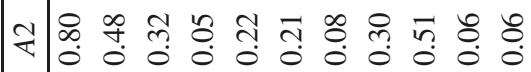

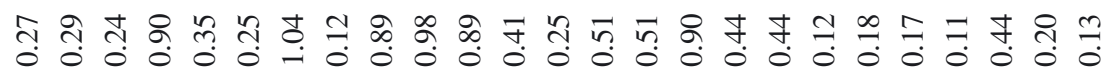

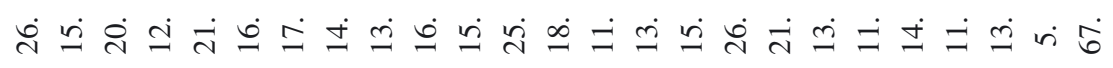

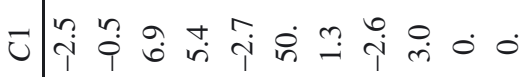

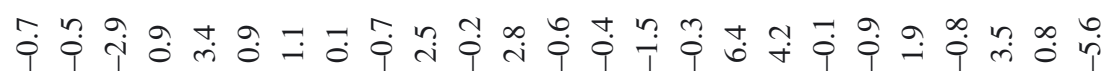

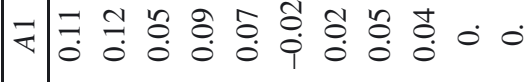

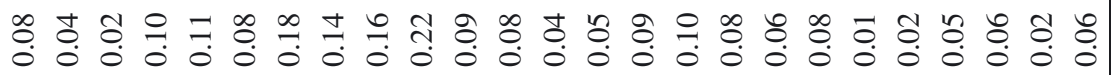

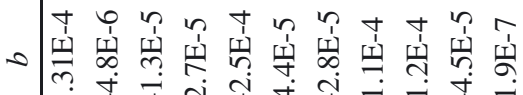

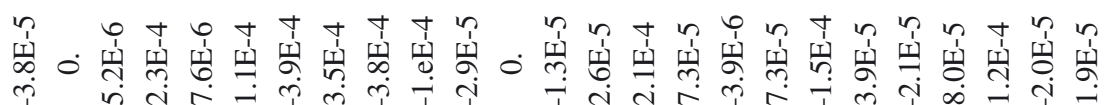
-

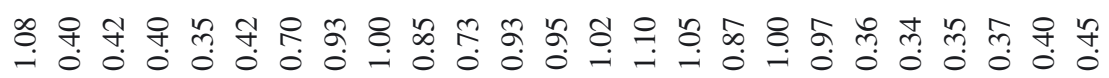

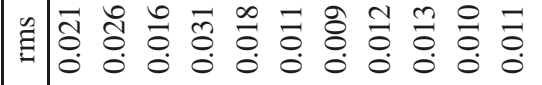

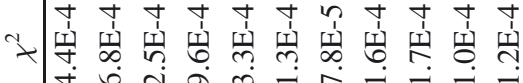

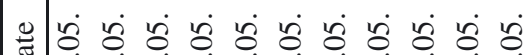

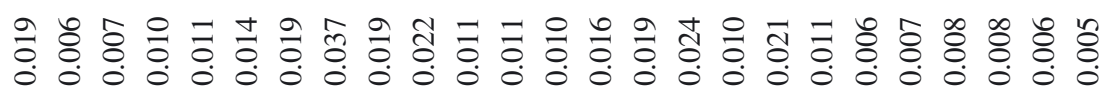

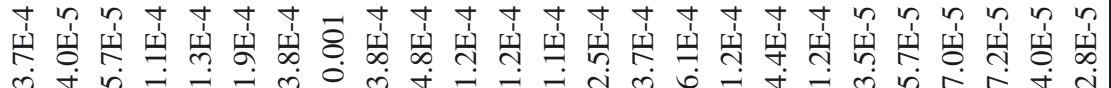

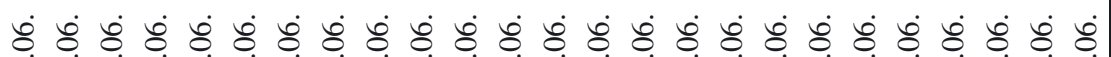

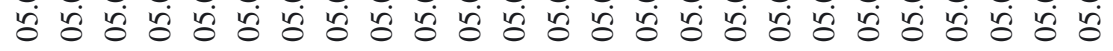

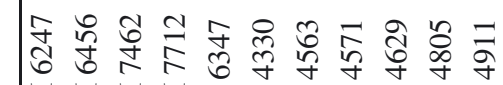
$\because \exists \exists \exists \exists \exists \exists \exists \exists$

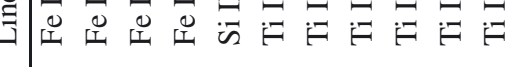

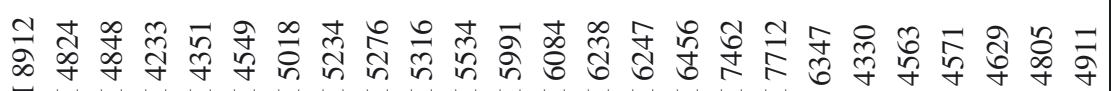

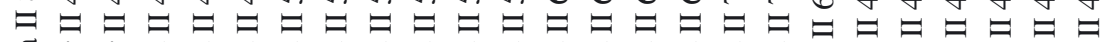
ฮ 


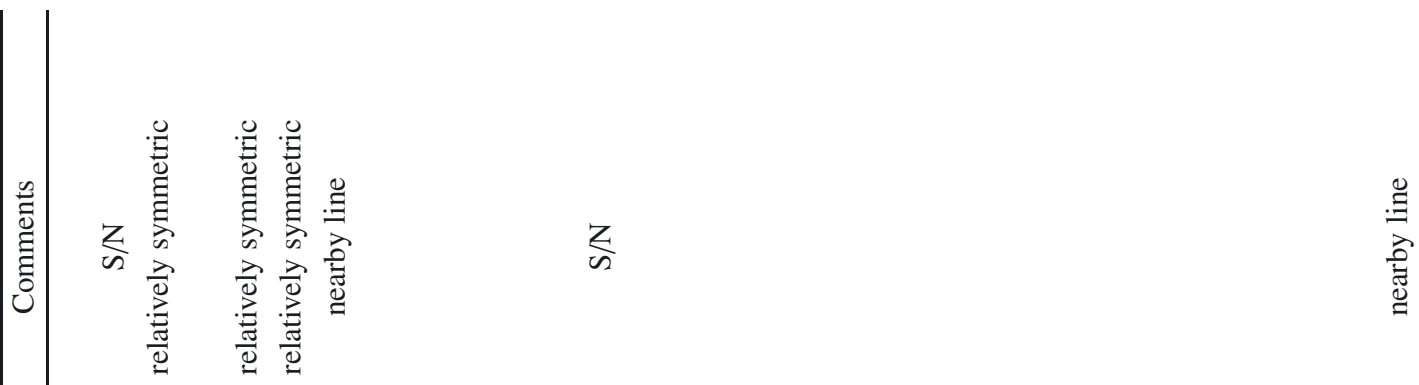

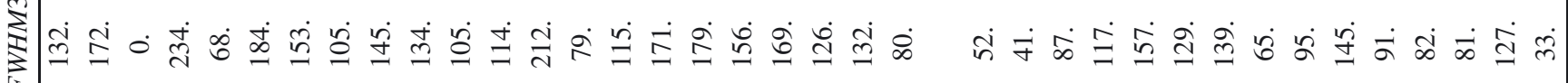
U ठं

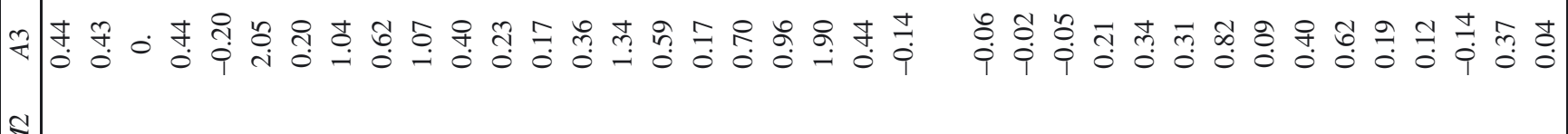

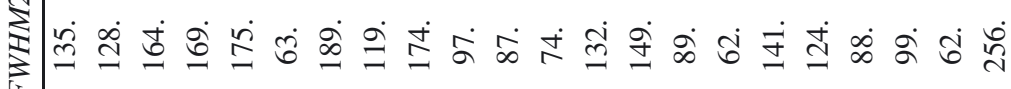

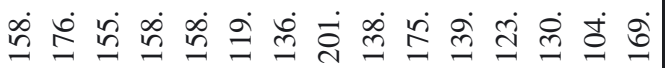
ป็น์

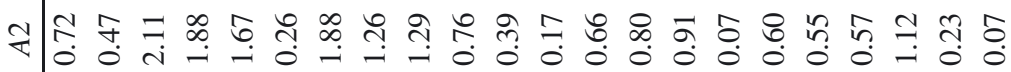

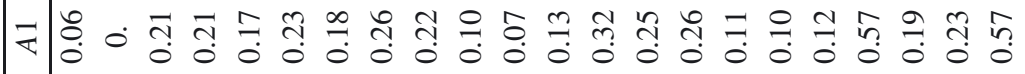

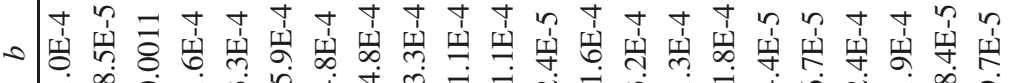

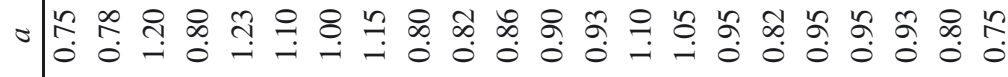

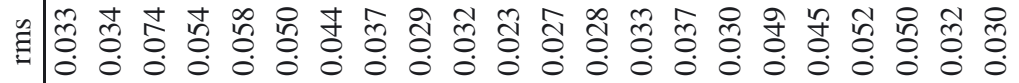

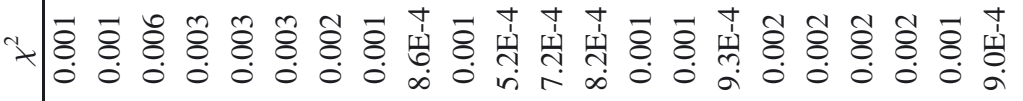

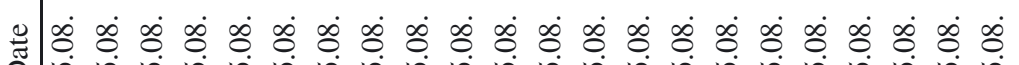

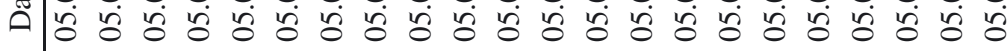

莳京

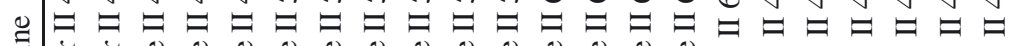

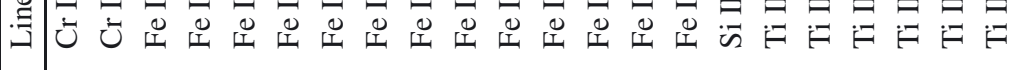
品 궁

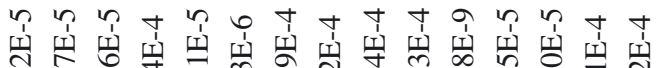
ति

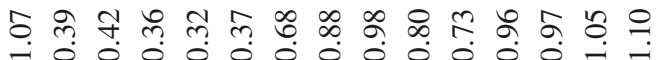

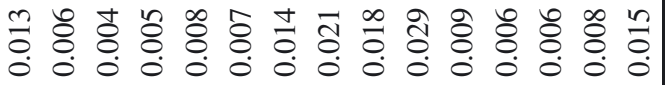

ம

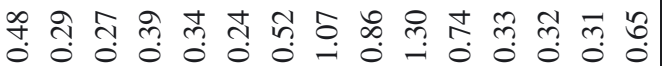

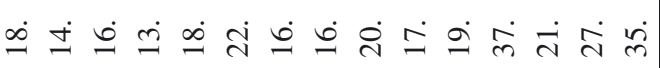

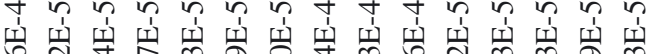

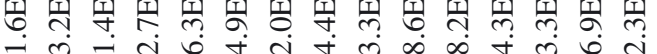

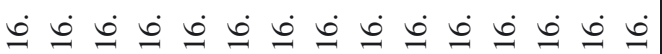
8ி

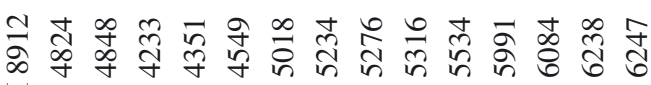

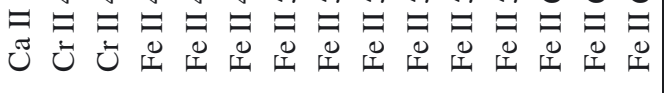




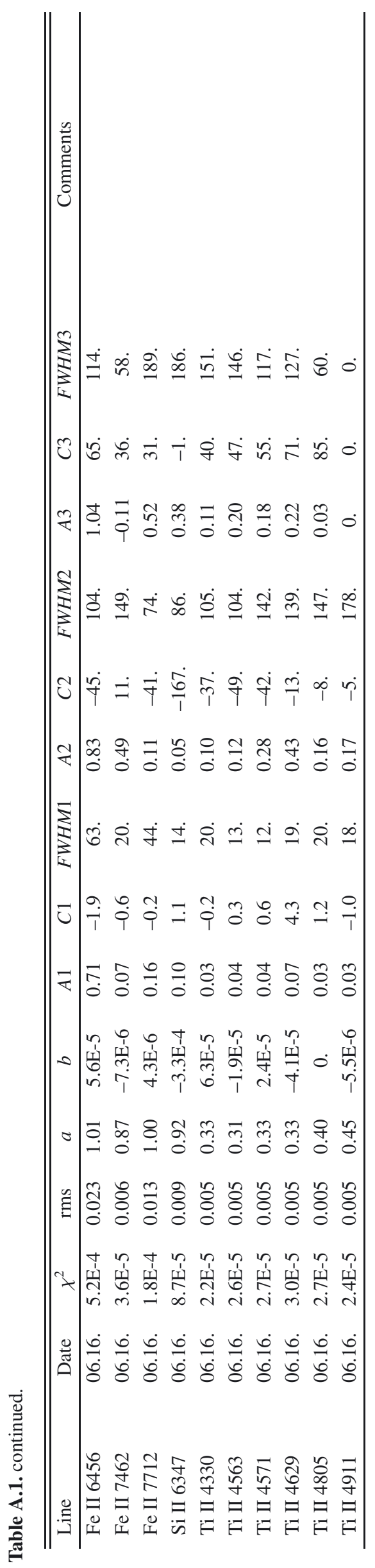

